\title{
The genus Pinnularia (Bacillariophyta, Pinnulariaceae) from Lago dos Tigres, Britânia, Goiás, Brazil
}

\author{
Weliton José da Silva ${ }^{1,2,4}$, Daiane Ruwer', Ina Nogueira ${ }^{2}$ \& Bárbara Dunck ${ }^{3}$ \\ ${ }^{1}$ Universidade Estadual de Londrina, Centro de Ciências Biológicas, Departamento de Biologia Animal e \\ Vegetal, Londrina, PR, Brazil. \\ ${ }^{2}$ Universidade Federal de Goiás, Instituto de Ciências Biológicas, Departamento de Botânica, Goiânia, GO, Brazil. \\ ${ }^{3}$ Universidade Estadual de Maringá, Programa de Pós-graduação em Ecologia de Ambientes Aquáticos \\ Continentais, Maringá, PR, Brazil. \\ ${ }^{4}$ Corresponding author: Weliton José da Silva, e-mail: welitondasilva@yahoo.com.br
}

DA SILVA, W.J., RUWER, D., NOGUEIRA, I., DUNCK, B. The genus Pinnularia (Bacillariophyta, Pinnulariaceae) from Lago dos Tigres, Britânia, Goiás, Brazil. Biota Neotropica. 16(1): e20150028. http:// dx.doi.org/10.1590/1676-0611-BN-2015-0028

\begin{abstract}
Studies of the genus Pinnularia Ehrenb. are only incipient in central-western Brazil, especially in Goiás. Only 20 of the 170 taxa known from Brazil were recorded in this state until now. A taxonomic study of Pinnularia from Lago dos Tigres (Tocantins-Araguaia River Basin) recorded 20 specific and infraspecific taxa, distributed in 17 species, including $P$. instabilis whose lectotype is designated here. Only P. meridiana var. meridiana was previously recorded in aquatic systems of the state. Eighteen taxa are newly recorded for Goiás, and one is the first record in Brazil. The Pinnularia flora from Lago dos Tigres contains nine taxa in common with the Amazon River Basin flora, and seven other taxa that occur elsewhere in the Paraná River Basin. Keywords: blocked river valley, Pinnularia meridian, Pinnularia instabilis, lectotypification, central-western Brazil.
\end{abstract}

DA SILVA, W.J., RUWER, D., NOGUEIRA, I., DUNCK, B. O gênero Pinnularia (Bacillariophyta, Pinnulariaceae) do Lago dos Tigres, Britânia, Goiás, Brasil. Biota Neotropica. 16(1): e20150028. http:// dx.doi.org/10.1590/1676-0611-BN-2015-0028

\begin{abstract}
Resumo: O gênero Pinnularia Ehrenb. é incipientemente estudado na região Centro-Oeste brasileira, especialmente em Goiás. Somente 20 de 170 táxons conhecidos no Brasil foram registrados nesse estado até agora. Nesse estudo, objetivou-se a realização de estudos taxonômicos de Pinnularia do Lago dos Tigres (Bacia Tocantins-Araguaia). Vinte táxons em nível específico e infraespecífico, distribuídos em 17 espécies, foram observados e caracterizados aqui, incluindo $P$. instabilis cujo lectótipo foi designado aqui. Somente $P$. meridiana var. meridiana foi primeiramente registrada em sistemas aquáticos do estado. Dezoito táxons constituíram citações pioneiras para o estado de Goiás e um foi o primeiro registro de ocorrência para o Brasil. A flora de Pinnularia do Lago dos Tigres apresentou nove táxons comuns com a Bacia Amazônica e outras sete foram comuns à Bacia do Paraná.
\end{abstract}

Palavras-chave: vale bloqueado, Pinnularia meridian, Pinnularia instabilis, lecotipificação, Centro-Oeste.

\section{Introduction}

The taxon name Pinnularia was proposed by Ehrenberg (1840a, 1840b) as a subgenus of Navicula Bory. Later, Ehrenberg (1843a, 1843b) used the name at generic level. However, the name Pinnularia was earlier used by Lindley \& Hutton (1835) for a genus of fossil high plants. Pinnularia Lindley \& Hutton was lesser used than Pinnularia Ehrenb., which was conserved against the first one and it is the correct name to generic level according the International Code for Nomenclature of algae, fungi and plants (ICN, McNeill et al. 2012).

Pinnularia is a genus of naviculoid diatoms characterized by valves linear, lanceolate or elliptic, with plane or undulate surfaces (Krammer 2000). Members of this genus usually presented different courses of the internal and external fissure of the raphe; transapically, the valves bear alveolated costal systems, which are simple or partially recovered (Krammer 2000). This genus occurs predominantly in oligotrophic continental waters, with low electrolyte concentration and low pH values (Uherkovich 1984, Metzeltin \& Lange-Bertalot 1998, Krammer 2000).

The genus Pinnularia comprises more than 2,700 species specific and infraspecific combinations, of which about 674 are currently accepted (Guiry \& Guiry 2015). In Brazil, studies about Pinnularia recorded approximately 215 taxa (Pereira et al. 2014, Eskinazi-Leça et al. 2016). These studies were mainly concentrated in the regions South (Torgan et al. 1999, Tremarin et al. 2009, 2010), Southeast (Menezes \& Dias 2001, Rocha \& Bicudo 2008) and North (Hustedt 1965, Metzeltin \& Lange-Bertalot 1998, 2007, Pereira et al. 2012, 2013, 2014).

In Central-West Region only 20 taxa were registered until now (Delgado \& Souza 2007, da Silva et al. 2011). These studies comprised small areas, such as Distrito Federal, the 
regions Central, Northeast, and Southwest from Goiás, and a small city in Mato Grosso. The Central-West, Brazil, bears sources from the main hydrographic regions from the country. Three of these are present in the State of Goiás (i.e., São Francisco, Paraná, and Tocantins-Araguaia). Nowadays, this region has undergoing to deforestation and biocide effects because agricultural practices, which have been listed as one of the main reasons of aquatic biodiversity loss (Tundisi 2003). This impels a race against time in order to know the diversity of algal organisms from areas in similar conditions.

Phylocogical studies in the Western region from Goiás are still scarce. The Lago dos Tigres is localized in this area and it has four studies that approaching the algal biodiversity (i.e., Nabout \& Nogueira 2007, Dunck et al. 2012, Oliveira et al. 2012a, 2012b), but none of them recorded species of Pinnularia. We aimed here to inventory the species of Pinnularia from Lago dos Tigres, Britânia, Goiás, Brazil.

\section{Material and methods}

Lago dos Tigres is localized in Britânia, in the West Region from the State of Goiás, Brazil (Figure 1). Commonly called lake, the Lago dos Tigres is, actually, characterized as blocked valley (Nabout \& Nogueira 2007), also called drowned valley. Drowned valleys can be originated from the natural damming of water volumes of receptor rivers or by deposition of sediments, creating a barrier to the water (Kalff 2002). In the Lago dos Tigres, the Água Limpa River dammed by the water of the Vermelho River and sediments deposited at the confluence of these two rivers, resulting in a lentic body $50 \mathrm{~km}^{2}$ of superficial area (Nabout \& Nogueira 2007). These systems constitute affluents of the Tocantins-Araguaia River Basin.
This Lago dos Tigres System has acid to neutral waters, which reach temperatures higher than $21{ }^{\circ} \mathrm{C}$. These waters are characterized as oligomesotrophic to mesotrophic, with high oxygen concentration (Nabout \& Nogueira 2007). Physical and chemical variables were measured and values of averages and standard deviations $(n=11)$ are provided in Table 1. Depth and water transparency were measured by means measuring tape and Secchi disk, respectively. Water temperature, conductivity, oxygen saturation and $\mathrm{pH}$ were determined through a water multi-analyzer (HORIBA U-21). Total nitrogen (TN) and total phosphorus (TP) were inferred according to APHA (2005).

Phytoplankton samples were obtained using $25 \mu \mathrm{m}$ plankton net. Periphytic material was scraped from artificial substrates (rocks) using toothbrush. These substrates were sampled after the $21^{\text {st }}$ day of colonization. The material examined was collected from the 11 sites along Lago dos Tigres system (Britânia, Goiás), monthly, from June 2004 to November 2004, and from June 2008 and January 2009, totalizing 178 samples (Table 2). All the samples were fixed with Transeau solution (Bicudo \& Menezes 2006) and deposited in the Herbarium of the Universidade Federal de Goiás (UFG), with numbers between UFG29864 and UFG29973, and between UFG43636 and UFG43724 (Table 2).

The samples were oxidized according to Simonsen (1974), modified by Moreira-Filho \& Valente-Moreira (1981) and permanent slides were mounted using Naphrax ${ }^{\circledR}$. The material was examined with Zeiss Axioscop 40 microscope and images were captured with an Axiovison system for observation of morphological characteristics of each taxa. A part of oxidized material was deposited under stubs and sputtered with a layer of 150-200 $\AA$ of gold in Sputter Coater Desk V (Denton Vacuum, LLC). The preparations were analyzed in a Jeol

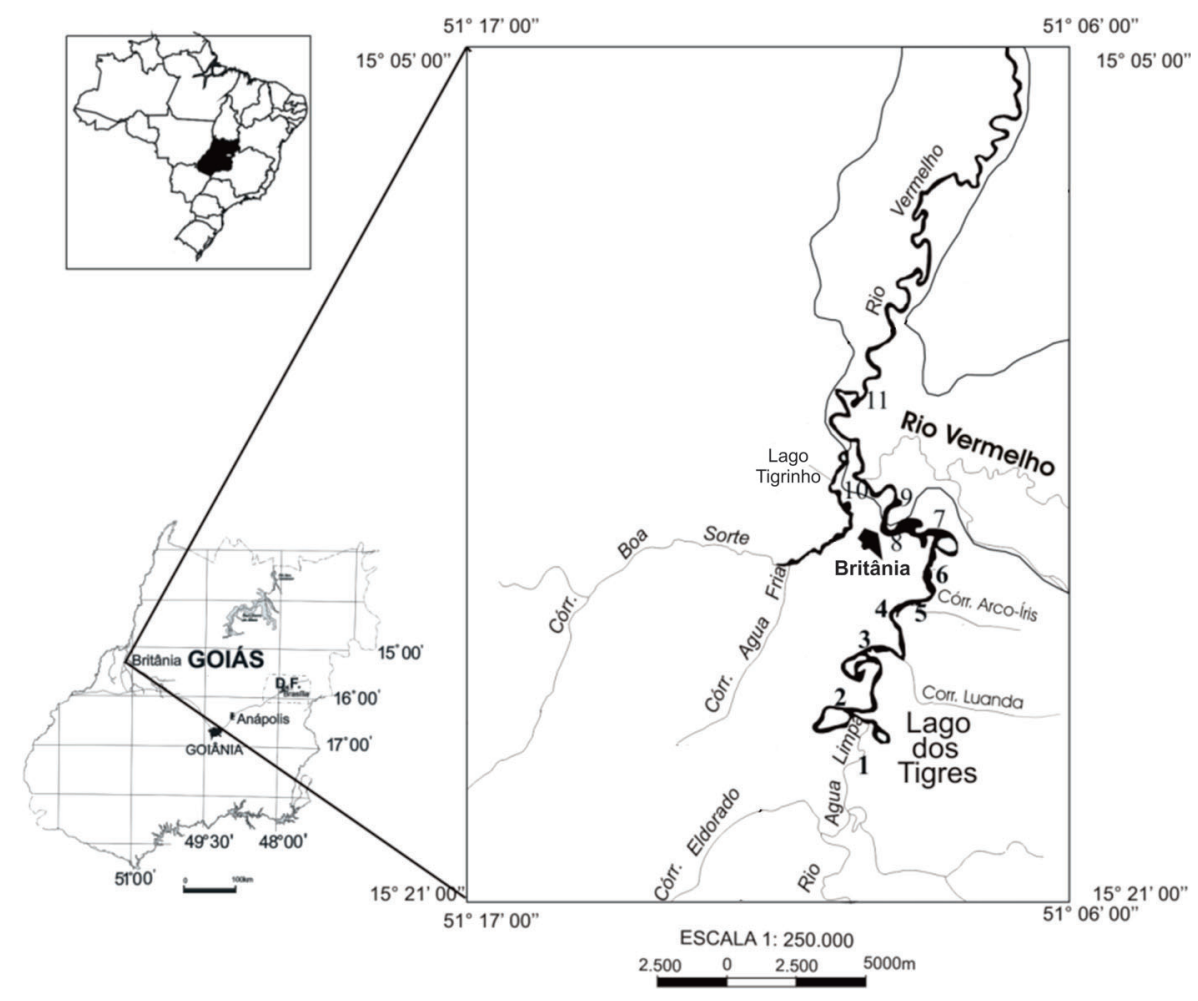

Figure 1. Lago dos Tigres System, Britânia, Goiás, and sampling sites in the study area. 


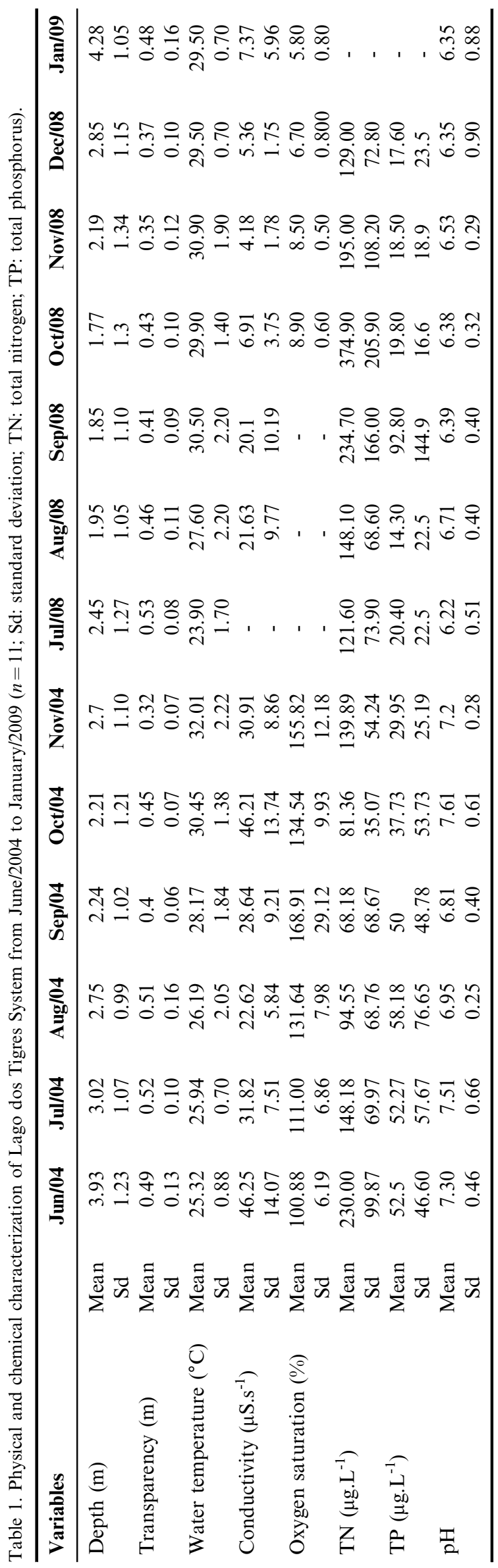

JSM-6610 scanning electron microscope (Jeol, USA), with an electrical potential of $6 \mathrm{kV}$, spot size 25-40.

The identification of the taxa at infrageneric level was based on specific bibliography and, as well as possible, based on original material (e.g., Metzeltin \& Lange-Bertalot 1998, 2007, Krammer 2000, Metzeltin et al. 2005). The standardization of the author names were made according to IPNI (http://www. ipni.org/).

The frequency of occurrence of each species in the samples was based on Guille (1970), according to which taxa with frequencies up to $10 \%$ were considered rare, between $10 \%$ and $50 \%$, inclusive, were considered common, and higher than $50 \%$ were considered constant.

\section{Results and discussion}

Twenty taxa of Pinnularia were recorded in this study (Table 3), all of them were considered rare according to the criteria proposed by Guille (1970) because occurred in $0.56 \%$ to $5.61 \%$ of the 178 samples analyzed in this study. The most frequent were $P$. meridiana var. meridiana Metzeltin \& Krammer and $P$. rumrichiae Krammer, which occurred in nine and ten samples, respectively. Only $P$. meridiana var. meridiana was recorded early in other diatomoflora study from the State of Goiás (Souza \& Oliveira 2007, da Silva et al. 2011). Other 18 taxa were firstly recorded in this State from this study, and Pinnularia microstauron var. rostrata constituted the first report for Brazil. Among the 18 taxa already recorded in Brazil, nine were common to environments from the Amazonas River Basin (Hustedt 1965, Simonsen 1987, Metzeltin \& Lange-Bertalot 1998, 2007, Pereira et al. 2012, 2013); seven occurred in states of São Paulo and or Paraná, from the Paraná River Basin (Rocha \& Bicudo 2008, Souza \& Senna 2009, Moresco et al. 2011); six taxa were common to the Guaraguaçu River Basin, in the State of Paraná (Tremarin et al. 2009, 2010, Santos et al. 2011); and one taxon were common to the Guaíba River Basin, in Rio Grande do Sul (Metzeltin \& Lange-Bertalot 1998). The only that already was recorded in Goiás also belongs to the Tocantins-Araguaia River Basin, to which run the waters of the Lago dos Tigres System (Souza \& Oliveira 2007, da Silva et al. 2011).

\section{Pinnularia acrosphaeria var. tumidula}

Krammer, Diatoms of Europe, vol. 1, p. 55, 214, Fig. 21: 8, 9, 2000. (Figure 2)

Holotype. Preparation 1792 MR, Collection Mayer in Regensburgische Botanische Gesellschaft (REG), Regensburg, Germany.

Type locality. Nelubium pond, in the botanical garden from Munich, Germany.

Valves linear, tumid at the middle part; broadly rounded apices; length: 79-82 $\mu \mathrm{m}$, breadth: 12.5-12.66 $\mu \mathrm{m}$, length/ breadth ratio: 6.32-6.57; axial area broad, linear, presenting granulations; raphe lateral, one terminal end abruptly curve and other sickle-shaped, proximal ends slightly curved to the same direction; striae parallel, becoming slightly radiate towards to the ends of the valves; striae: $12 / 10 \mu \mathrm{m}$.

Material examined. Brazil, State of Goiás, Britânia, Lago dos Tigres, plankton, Nogueira et al. Site 01, 19/10/2008, P01M10, ISN1265 (UFG 43675); Site 4, 04/06/2004, ISN877 (UFG 29866); Rio Vermelho, plankton, Nogueira et al. Site 11, 14/07/ 2004, ISN907 (UFG 29890). 
Table 2. Samples collected in the Lago dos Tigres Systems

\begin{tabular}{|c|c|c|c|c|c|c|}
\hline $\begin{array}{l}\text { Herbarium } \\
\text { number }\end{array}$ & Locality & Date & Coordinates & $\begin{array}{l}\text { Collector } \\
\text { number }\end{array}$ & Collectors & Habitat \\
\hline UFG29864 & $\begin{array}{l}\text { Brazil, State of Goiás, Britânia, } \\
\text { Água Limpa River, Água } \\
\text { Limpa Farm, Site } 1\end{array}$ & $04 / 06 / 2004$ & $\begin{array}{l}15^{\circ} 18^{\prime} 58^{\prime \prime} \mathrm{S} ; \\
51^{\circ} 9^{\prime} 56^{\prime \prime} \mathrm{W}\end{array}$ & ISN874 & $\begin{array}{l}\text { Nogueira, I.S., Martins, } \\
\text { L. L., da Silva, W.J., } \\
\text { Nabout, J.C. }\end{array}$ & Phytoplankton \\
\hline UFG29865 & $\begin{array}{l}\text { Brazil, State of Goiás, } \\
\text { Britânia, Lago dos Tigres, } \\
\text { Perdidas Island, Site } 2\end{array}$ & $04 / 06 / 2004$ & $\begin{array}{l}15^{\circ} 17^{\prime} 57^{\prime \prime} \mathrm{S} ; \\
51^{\circ} 10^{\prime} 7^{\prime \prime} \mathrm{W}\end{array}$ & ISN876 & $\begin{array}{l}\text { Nogueira, I.S., Martins, } \\
\text { L. L., da Silva, W.J., } \\
\text { Nabout, J.C. }\end{array}$ & Phytoplankton \\
\hline UFG29866 & $\begin{array}{l}\text { Brazil, State of Goiás, } \\
\text { Britânia, Lago dos Tigres, } \\
\text { Santo Antônio Farm, Site } 4\end{array}$ & $04 / 06 / 2004$ & $\begin{array}{l}15^{\circ} 16^{\prime} 17^{\prime \prime} \mathrm{S} ; \\
51^{\circ} 9^{\prime} 10^{\prime \prime} \mathrm{W}\end{array}$ & ISN877 & $\begin{array}{l}\text { Nogueira, I.S., Martins, } \\
\text { L. L., da Silva, W.J., } \\
\text { Nabout, J.C. }\end{array}$ & Phytoplankton \\
\hline UFG29867 & $\begin{array}{l}\text { Brazil, State of Goiás, } \\
\text { Britânia, Lago dos Tigres, } \\
\text { harbor ferry, Site } 6\end{array}$ & $04 / 06 / 2004$ & $\begin{array}{l}15^{\circ} 15^{\prime} 12^{\prime \prime} \mathrm{S} ; \\
51^{\circ} 8^{\prime} 33^{\prime \prime} \mathrm{W}\end{array}$ & ISN878 & $\begin{array}{l}\text { Nogueira, I.S., Martins, } \\
\text { L. L., da Silva, W.J., } \\
\text { Nabout, J.C. }\end{array}$ & Phytoplankton \\
\hline UFG29868 & $\begin{array}{l}\text { Brazil, State of Goiás, } \\
\text { Britânia, Lago dos Tigres, } \\
\text { Lago dos Tigres Farm, Site } 7\end{array}$ & $04 / 06 / 2004$ & $\begin{array}{l}15^{\circ} 14^{\prime} 17^{\prime \prime} \mathrm{S} ; \\
51^{\circ} 8^{\prime} 59^{\prime \prime} \mathrm{W}\end{array}$ & ISN879 & $\begin{array}{l}\text { Nogueira, I.S., Martins, } \\
\text { L. L., da Silva, W.J., } \\
\text { Nabout, J.C. }\end{array}$ & Phytoplankton \\
\hline UFG29869 & $\begin{array}{l}\text { Brazil, State of Goiás, } \\
\text { Britânia, Lago dos Tigres, city } \\
\text { in front of the Christ, Site } 8\end{array}$ & $04 / 06 / 2004$ & $\begin{array}{l}15^{\circ} 14^{\prime} 9^{\prime \prime} \mathrm{S} \\
51^{\circ} 9^{\prime} 27^{\prime \prime} \mathrm{W}\end{array}$ & ISN869 & $\begin{array}{l}\text { Nogueira, I.S., Martins, } \\
\text { L. L., da Silva, W.J., } \\
\text { Nabout, J.C. }\end{array}$ & Phytoplankton \\
\hline UFG29870 & $\begin{array}{l}\text { Brazil, State of Goiás, } \\
\text { Britânia, Lago dos Tigres, } \\
\text { outfall of the "lake" in } \\
\text { Vermelho River, Site } 10\end{array}$ & $04 / 06 / 2004$ & $\begin{array}{l}15^{\circ} 13^{\prime} 18^{\prime \prime} \mathrm{S} ; \\
51^{\circ} 10^{\prime} 6^{\prime \prime} \mathrm{W}\end{array}$ & ISN881 & $\begin{array}{l}\text { Nogueira, I.S., Martins, } \\
\text { L. L., da Silva, W.J., } \\
\text { Nabout, J.C. }\end{array}$ & Phytoplankton \\
\hline UFG29871 & $\begin{array}{l}\text { Brazil, State of Goiás, } \\
\text { Britânia, Lago dos Tigres, } \\
\text { new channel of the Vermelho } \\
\text { River, Site } 11\end{array}$ & $04 / 06 / 2004$ & $\begin{array}{l}15^{\circ} 11^{\prime} 47^{\prime \prime} \mathrm{S} ; \\
51^{\circ} 9^{\prime} 57^{\prime \prime} \mathrm{W}\end{array}$ & ISN882 & $\begin{array}{l}\text { Nogueira, I.S., Martins, } \\
\text { L. L., da Silva, W.J., } \\
\text { Nabout, J.C. }\end{array}$ & Phytoplankton \\
\hline UFG29872 & $\begin{array}{l}\text { Brazil, State of Goiás, } \\
\text { Britânia, Água Limpa River, } \\
\text { Água Limpa Farm, Site 1, } \\
\text { under macrophytes }\end{array}$ & $04 / 06 / 2004$ & $\begin{array}{l}15^{\circ} 18^{\prime} 58^{\prime \prime} \mathrm{S} \\
51^{\circ} 9^{\prime} 56^{\prime \prime} \mathrm{W}\end{array}$ & ISN889 & $\begin{array}{l}\text { Nogueira, I.S., Martins, } \\
\text { L.L., da Silva, W.J., } \\
\text { Nabout, J.C. }\end{array}$ & Periphyton \\
\hline UFG 29873 & $\begin{array}{l}\text { Brazil, State of Goiás, } \\
\text { Britânia, Lago dos Tigres, } \\
\text { harbor ferry, Site } 6\end{array}$ & $04 / 06 / 2004$ & $\begin{array}{l}15^{\circ} 15^{\prime} 12^{\prime \prime} \mathrm{S} ; \\
51^{\circ} 8^{\prime} 33^{\prime \prime} \mathrm{W}\end{array}$ & ISN875 & $\begin{array}{l}\text { Nogueira, I.S., Martins, } \\
\text { L.L., da Silva, W.J., } \\
\text { Nabout, J.C. }\end{array}$ & Periphyton \\
\hline UFG29875 & $\begin{array}{l}\text { Brazil, State of Goiás, } \\
\text { Britânia, Lago dos Tigres, } \\
\text { outfall of Arco-Íris Stream, } \\
\text { Site } 5\end{array}$ & $05 / 06 / 2004$ & $\begin{array}{l}15^{\circ} 15^{\prime} 46^{\prime \prime} \mathrm{S} \\
51^{\circ} 8^{\prime} 41^{\prime \prime} \mathrm{W}\end{array}$ & ISN891 & $\begin{array}{l}\text { Nogueira, I.S., Martins, } \\
\text { L.L., da Silva, W.J., } \\
\text { Nabout, J.C. }\end{array}$ & Periphyton \\
\hline UFG29876 & $\begin{array}{l}\text { Brazil, State of Goiás, } \\
\text { Britânia, Lago dos Tigres, city } \\
\text { in front of the Christ, Site } 8\end{array}$ & $05 / 06 / 2004$ & $\begin{array}{l}15^{\circ} 14^{\prime} 9^{\prime \prime} \mathrm{S} \\
51^{\circ} 9^{\prime} 27^{\prime \prime} \mathrm{W}\end{array}$ & ISN892 & $\begin{array}{l}\text { Nogueira, I.S., Martins, } \\
\text { L.L., da Silva, W.J., } \\
\text { Nabout, J.C. }\end{array}$ & Periphyton \\
\hline UFG29877 & $\begin{array}{l}\text { Brazil, State of Goiás, } \\
\text { Britânia, Lago dos Tigres, } \\
\text { outfall of the "lake" in } \\
\text { Vermelho River, Site } 10\end{array}$ & $05 / 06 / 2004$ & $\begin{array}{l}15^{\circ} 13^{\prime} 18^{\prime \prime} \mathrm{S} ; \\
51^{\circ} 10^{\prime} 6^{\prime \prime} \mathrm{W}\end{array}$ & ISN893 & $\begin{array}{l}\text { Nogueira, I.S., Martins, } \\
\text { L.L., da Silva, W.J., } \\
\text { Nabout, J.C. }\end{array}$ & Periphyton \\
\hline UFG29878 & $\begin{array}{l}\text { Brazil, State of Goiás, } \\
\text { Britânia, Lago dos Tigres, } \\
\text { new channel of the Vermelho } \\
\text { River, Site } 11\end{array}$ & $05 / 06 / 2004$ & $\begin{array}{l}15^{\circ} 11^{\prime} 47^{\prime \prime} \mathrm{S} ; \\
51^{\circ} 9^{\prime} 57^{\prime \prime} \mathrm{W}\end{array}$ & ISN894 & $\begin{array}{l}\text { Nogueira, I.S., Martins, } \\
\text { L.L., da Silva, W.J., } \\
\text { Nabout, J.C. }\end{array}$ & Periphyton \\
\hline UFG 29880 & $\begin{array}{l}\text { Brazil, State of Goiás, } \\
\text { Britânia, Água Limpa River, } \\
\text { Água Limpa Farm, Site } 1\end{array}$ & $13 / 07 / 2004$ & $\begin{array}{l}15^{\circ} 18^{\prime} 58^{\prime \prime} \mathrm{S} ; \\
51^{\circ} 9^{\prime} 56^{\prime \prime} \mathrm{W}\end{array}$ & ISN898 & $\begin{array}{l}\text { Nogueira, I.S., da } \\
\text { Silva, W.J., Nabout, } \\
\text { J.C., Oliveira, J.E., } \\
\text { Jorge, V.A. }\end{array}$ & Phytoplankton \\
\hline UFG29881 & $\begin{array}{l}\text { Brazil, State of Goiás, } \\
\text { Britânia, Lago dos Tigres, } \\
\text { Perdidas Island, Site } 2\end{array}$ & $13 / 07 / 2004$ & $\begin{array}{l}15^{\circ} 17^{\prime} 57^{\prime \prime} \mathrm{S} ; \\
51^{\circ} 10^{\prime} 7^{\prime \prime} \mathrm{W}\end{array}$ & ISN899 & $\begin{array}{l}\text { Nogueira, I.S., da } \\
\text { Silva, W.J., Nabout, } \\
\text { J.C., Oliveira, J.E., } \\
\text { Jorge, V.A. }\end{array}$ & Phytoplankton \\
\hline
\end{tabular}


Table 2. Continued.

\begin{tabular}{|c|c|c|c|c|c|c|}
\hline $\begin{array}{l}\text { Herbarium } \\
\text { number }\end{array}$ & Locality & Date & Coordinates & $\begin{array}{l}\text { Collector } \\
\text { number }\end{array}$ & Collectors & Habitat \\
\hline UFG29882 & $\begin{array}{l}\text { Brazil, State of Goiás, } \\
\text { Britânia, Lago dos Tigres, } \\
\text { outfall of Luanda Stream, } \\
\text { Santo Antônio Farm, Site } 3\end{array}$ & $13 / 07 / 2004$ & $\begin{array}{l}15^{\circ} 16^{\prime} 47^{\prime \prime} \mathrm{S} ; \\
51^{\circ} 9^{\prime} 44^{\prime \prime} \mathrm{W}\end{array}$ & ISN900 & $\begin{array}{l}\text { Nogueira, I.S., da } \\
\text { Silva, W.J., Nabout, } \\
\text { J.C., Oliveira, J.E., } \\
\text { Jorge, V.A. }\end{array}$ & Phytoplankton \\
\hline UFG29883 & $\begin{array}{l}\text { Brazil, State of Goiás, } \\
\text { Britânia, Lago dos Tigres, } \\
\text { Santo Antônio Farm, Site } 4\end{array}$ & $13 / 07 / 2004$ & $\begin{array}{l}15^{\circ} 16^{\prime} 17^{\prime \prime} \mathrm{S} \\
51^{\circ} 9^{\prime} 10^{\prime \prime} \mathrm{W}\end{array}$ & ISN901 & $\begin{array}{l}\text { Nogueira, I.S., da } \\
\text { Silva, W.J., Nabout, } \\
\text { J.C., Oliveira, J.E., } \\
\text { Jorge, V.A. }\end{array}$ & Phytoplankton \\
\hline UFG29884 & $\begin{array}{l}\text { Brazil, State of Goiás, } \\
\text { Britânia, Lago dos Tigres, } \\
\text { outfall of Arco-Íris Stream, } \\
\text { Site } 5\end{array}$ & $13 / 07 / 2004$ & $\begin{array}{l}15^{\circ} 15^{\prime} 46^{\prime \prime} \mathrm{S} \\
51^{\circ} 8^{\prime} 41^{\prime \prime} \mathrm{W}\end{array}$ & ISN902 & $\begin{array}{l}\text { Nogueira, I.S., da } \\
\text { Silva, W.J., Nabout, } \\
\text { J.C., Oliveira, J.E., } \\
\text { Jorge, V.A. }\end{array}$ & Phytoplankton \\
\hline UFG29885 & $\begin{array}{l}\text { Brazil, State of Goiás, } \\
\text { Britânia, Lago dos Tigres, } \\
\text { outfall of the "lake" in } \\
\text { Vermelho River, Site } 9\end{array}$ & $13 / 07 / 2004$ & $\begin{array}{l}15^{\circ} 15^{\prime} 46^{\prime \prime} \mathrm{S} \\
51^{\circ} 8^{\prime} 41^{\prime \prime} \mathrm{W}\end{array}$ & ISN913 & $\begin{array}{l}\text { Nogueira, I.S., da } \\
\text { Silva, W.J., Nabout, } \\
\text { J.C., Oliveira, J.E., } \\
\text { Jorge, V.A. }\end{array}$ & Phytoplankton \\
\hline UFG29886 & $\begin{array}{l}\text { Brazil, State of Goiás, } \\
\text { Britânia, Lago dos Tigres, } \\
\text { harbor ferry, Site } 6\end{array}$ & $13 / 07 / 2004$ & $\begin{array}{l}15^{\circ} 15^{\prime} 12^{\prime \prime} \mathrm{S} ; \\
51^{\circ} 8^{\prime} 33^{\prime \prime} \mathrm{W}\end{array}$ & ISN903 & $\begin{array}{l}\text { Nogueira, I.S., da } \\
\text { Silva, W.J., Nabout, } \\
\text { J.C., Oliveira, J.E., } \\
\text { Jorge, V.A. }\end{array}$ & Phytoplankton \\
\hline UFG29887 & $\begin{array}{l}\text { Brazil, State of Goiás, } \\
\text { Britânia, Lago dos Tigres, } \\
\text { Lago dos Tigres Farm, Site } 7\end{array}$ & $13 / 07 / 2004$ & $\begin{array}{l}15^{\circ} 14^{\prime} 17^{\prime \prime} \mathrm{S} \\
51^{\circ} 8^{\prime} 59^{\prime \prime} \mathrm{W}\end{array}$ & ISN904 & $\begin{array}{l}\text { Nogueira, I.S., da } \\
\text { Silva, W.J., Nabout, } \\
\text { J.C., Oliveira, J.E., } \\
\text { Jorge, V.A. }\end{array}$ & Phytoplankton \\
\hline UFG29888 & $\begin{array}{l}\text { Brazil, State of Goiás, } \\
\text { Britânia, Lago dos Tigres, city } \\
\text { in front of the Christ, Site } 8\end{array}$ & $13 / 07 / 2004$ & $\begin{array}{l}15^{\circ} 14^{\prime} 9^{\prime \prime} \mathrm{S} \\
51^{\circ} 9^{\prime} 27^{\prime \prime} \mathrm{W}\end{array}$ & ISN905 & $\begin{array}{l}\text { Nogueira, I.S., da } \\
\text { Silva, W.J., Nabout, } \\
\text { J.C., Oliveira, J.E., } \\
\text { Jorge, V.A. }\end{array}$ & Phytoplankton \\
\hline UFG29889 & $\begin{array}{l}\text { Brazil, State of Goiás, } \\
\text { Britânia, Lago dos Tigres, } \\
\text { downstream of the "lake", } \\
\text { Lago dos Tigres Farm, Site } 9\end{array}$ & $14 / 07 / 2004$ & $\begin{array}{l}15^{\circ} 13^{\prime} 43^{\prime \prime} \mathrm{S} \\
51^{\circ} 9^{\prime} 14^{\prime \prime} \mathrm{W}\end{array}$ & ISN906 & $\begin{array}{l}\text { Nogueira, I.S., da } \\
\text { Silva, W.J., Nabout, } \\
\text { J.C., Oliveira, J.E., } \\
\text { Jorge, V.A. }\end{array}$ & Phytoplankton \\
\hline UFG29890 & $\begin{array}{l}\text { Brazil, State of Goiás, } \\
\text { Britânia, Lago dos Tigres, } \\
\text { outfall of the "lake" in } \\
\text { Vermelho River, Site } 10\end{array}$ & $14 / 07 / 2004$ & $\begin{array}{l}15^{\circ} 13^{\prime} 18^{\prime \prime} \mathrm{S} \\
51^{\circ} 10^{\prime} 6^{\prime \prime} \mathrm{W}\end{array}$ & ISN907 & $\begin{array}{l}\text { Nogueira, I.S., da } \\
\text { Silva, W.J., Nabout, } \\
\text { J.C., Oliveira, J.E., } \\
\text { Jorge, V.A. }\end{array}$ & Phytoplankton \\
\hline UFG29891 & $\begin{array}{l}\text { Brazil, State of Goiás, } \\
\text { Britânia, Lago dos Tigres, } \\
\text { new channel of the Vermelho } \\
\text { River, Site } 11\end{array}$ & $14 / 07 / 2004$ & $\begin{array}{l}15^{\circ} 11^{\prime} 47^{\prime \prime} \mathrm{S} ; \\
51^{\circ} 9^{\prime} 57^{\prime \prime} \mathrm{W}\end{array}$ & ISN908 & $\begin{array}{l}\text { Nogueira, I.S., da } \\
\text { Silva, W.J., Nabout, } \\
\text { J.C., Oliveira, J.E., } \\
\text { Jorge, V.A. }\end{array}$ & Phytoplankton \\
\hline UFG29895 & $\begin{array}{l}\text { Brazil, State of Goiás, } \\
\text { Britânia, Água Limpa River, } \\
\text { Água Limpa Farm, Site } 1\end{array}$ & $19 / 08 / 2004$ & $\begin{array}{l}15^{\circ} 18^{\prime} 58^{\prime \prime} \mathrm{S} \\
51^{\circ} 9^{\prime} 56^{\prime \prime} \mathrm{W}\end{array}$ & ISN945 & $\begin{array}{l}\text { Nogueira, I.S., da } \\
\text { Silva, W.J., Nabout, } \\
\text { J.C., Oliveira, J.E., } \\
\text { Jorge, V.A. }\end{array}$ & Phytoplankton \\
\hline UFG29896 & $\begin{array}{l}\text { Brazil, State of Goiás, } \\
\text { Britânia, Lago dos Tigres, } \\
\text { Perdidas Island, Site } 2\end{array}$ & $19 / 08 / 2004$ & $\begin{array}{l}15^{\circ} 17^{\prime} 57^{\prime \prime} \mathrm{S} \\
51^{\circ} 10^{\prime} 7^{\prime \prime} \mathrm{W}\end{array}$ & ISN946 & $\begin{array}{l}\text { Nogueira, I.S., } \\
\text { Martins, L. L., Uto, } \\
\text { F.N., Nabout, J.C. }\end{array}$ & Phytoplankton \\
\hline UFG29897 & $\begin{array}{l}\text { Brazil, State of Goiás, } \\
\text { Britânia, Lago dos Tigres, } \\
\text { outfall of Luanda Stream, } \\
\text { Santo Antônio Farm, Site } 3\end{array}$ & $19 / 08 / 2004$ & $\begin{array}{l}15^{\circ} 16^{\prime} 47^{\prime \prime} \mathrm{S} \\
51^{\circ} 9^{\prime} 44^{\prime \prime} \mathrm{W}\end{array}$ & ISN947 & $\begin{array}{l}\text { Nogueira, I.S., } \\
\text { Martins, L. L., Uto, } \\
\text { F.N., Nabout, J.C. }\end{array}$ & Phytoplankton \\
\hline UFG29898 & $\begin{array}{l}\text { Brazil, State of Goiás, } \\
\text { Britânia, Lago dos Tigres, } \\
\text { Santo Antônio Farm, Site } 4\end{array}$ & $19 / 08 / 2004$ & $\begin{array}{l}15^{\circ} 16^{\prime} 17^{\prime \prime} \mathrm{S} \\
51^{\circ} 9^{\prime} 10^{\prime \prime} \mathrm{W}\end{array}$ & ISN948 & $\begin{array}{l}\text { Nogueira, I.S., } \\
\text { Martins, L. L., Uto, } \\
\text { F.N., Nabout, J.C. }\end{array}$ & Phytoplankton \\
\hline
\end{tabular}


Table 2. Continued.

\begin{tabular}{|c|c|c|c|c|c|c|}
\hline $\begin{array}{l}\text { Herbarium } \\
\text { number }\end{array}$ & Locality & Date & Coordinates & $\begin{array}{l}\text { Collector } \\
\text { number }\end{array}$ & Collectors & Habitat \\
\hline UFG29899 & $\begin{array}{l}\text { Brazil, State of Goiás, } \\
\text { Britânia, Lago dos Tigres, } \\
\text { outfall of Arco-Íris Stream, } \\
\text { Site } 5\end{array}$ & $19 / 08 / 2004$ & $\begin{array}{l}15^{\circ} 15^{\prime} 46^{\prime \prime} \mathrm{S} \\
51^{\circ} 8^{\prime} 41^{\prime \prime} \mathrm{W}\end{array}$ & ISN949 & $\begin{array}{l}\text { Nogueira, I.S., } \\
\text { Martins, L. L., Uto, } \\
\text { F.N., Nabout, J.C. }\end{array}$ & Phytoplankton \\
\hline UFG29900 & $\begin{array}{l}\text { Brazil, State of Goiás, } \\
\text { Britânia, Lago dos Tigres, } \\
\text { harbor ferry, Site } 6\end{array}$ & $19 / 08 / 2004$ & $\begin{array}{l}15^{\circ} 15^{\prime} 12^{\prime \prime} \mathrm{S} \\
51^{\circ} 8^{\prime} 33^{\prime \prime} \mathrm{W}\end{array}$ & ISN950 & $\begin{array}{l}\text { Nogueira, I.S., } \\
\text { Martins, L. L., Uto, } \\
\text { F.N., Nabout, J.C. }\end{array}$ & Phytoplankton \\
\hline UFG29901 & $\begin{array}{l}\text { Brazil, State of Goiás, } \\
\text { Britânia, Lago dos Tigres, } \\
\text { Lago dos Tigres Farm, Site } 7\end{array}$ & $19 / 08 / 2004$ & $\begin{array}{l}15^{\circ} 14^{\prime} 17^{\prime \prime} \mathrm{S} ; \\
51^{\circ} 8^{\prime} 59^{\prime \prime} \mathrm{W}\end{array}$ & ISN951 & $\begin{array}{l}\text { Nogueira, I.S., } \\
\text { Martins, L. L., Uto, } \\
\text { F.N., Nabout, J.C. }\end{array}$ & Phytoplankton \\
\hline UFG29902 & $\begin{array}{l}\text { Brazil, State of Goiás, } \\
\text { Britânia, Lago dos Tigres, city } \\
\text { in front of the Christ, Site } 8\end{array}$ & $19 / 08 / 2004$ & $\begin{array}{l}15^{\circ} 14^{\prime} 9^{\prime \prime} \mathrm{S} ; \\
51^{\circ} 9^{\prime} 27^{\prime \prime} \mathrm{W}\end{array}$ & ISN952 & $\begin{array}{l}\text { Nogueira, I.S., } \\
\text { Martins, L. L., Uto, } \\
\text { F.N., Nabout, J.C. }\end{array}$ & Phytoplankton \\
\hline UFG29903 & $\begin{array}{l}\text { Brazil, State of Goiás, } \\
\text { Britânia, Lago dos Tigres, } \\
\text { downstream of the "lake", } \\
\text { Lago dos Tigres Farm, Site } 9\end{array}$ & $20 / 08 / 2004$ & $\begin{array}{l}15^{\circ} 13^{\prime} 43^{\prime \prime} \mathrm{S} ; \\
51^{\circ} 9^{\prime} 14^{\prime \prime} \mathrm{W}\end{array}$ & ISN953 & $\begin{array}{l}\text { Nogueira, I.S., } \\
\text { Martins, L. L., Uto, } \\
\text { F.N., Nabout, J.C. }\end{array}$ & Phytoplankton \\
\hline UFG29904 & $\begin{array}{l}\text { Brazil, State of Goiás, } \\
\text { Britânia, Lago dos Tigres, } \\
\text { outfall of the "lake" in } \\
\text { Vermelho River, Site } 10\end{array}$ & $20 / 08 / 2004$ & $\begin{array}{l}15^{\circ} 13^{\prime} 18^{\prime \prime} \mathrm{S} ; \\
51^{\circ} 10^{\prime} 6^{\prime \prime} \mathrm{W}\end{array}$ & ISN954 & $\begin{array}{l}\text { Nogueira, I.S., } \\
\text { Martins, L. L., Uto, } \\
\text { F.N., Nabout, J.C. }\end{array}$ & Phytoplankton \\
\hline UFG29905 & $\begin{array}{l}\text { Brazil, State of Goiás, } \\
\text { Britânia, Lago dos Tigres, } \\
\text { new channel of the Vermelho } \\
\text { River, Site } 11\end{array}$ & $20 / 08 / 2004$ & $\begin{array}{l}15^{\circ} 11^{\prime} 47^{\prime \prime} \mathrm{S} ; \\
51^{\circ} 9^{\prime} 57^{\prime \prime} \mathrm{W}\end{array}$ & ISN955 & $\begin{array}{l}\text { Nogueira, I.S., } \\
\text { Martins, L. L., Uto, } \\
\text { F.N., Nabout, J.C. }\end{array}$ & Phytoplankton \\
\hline UFG29907 & $\begin{array}{l}\text { Brazil, State of Goiás, } \\
\text { Britânia, Água Limpa River, } \\
\text { Água Limpa Farm, Site } 1\end{array}$ & 09/09/2004 & $\begin{array}{l}15^{\circ} 18^{\prime} 58^{\prime \prime} \mathrm{S} ; \\
51^{\circ} 9^{\prime} 56^{\prime \prime} \mathrm{W}\end{array}$ & ISN985 & $\begin{array}{l}\text { Nogueira, I.S., } \\
\text { Martins, L. L., da } \\
\text { Silva, W.J., } \\
\text { Rodrigues, J. }\end{array}$ & Phytoplankton \\
\hline UFG29908 & $\begin{array}{l}\text { Brazil, State of Goiás, } \\
\text { Britânia, Lago dos Tigres, } \\
\text { Perdidas Island, Site } 2\end{array}$ & 09/09/2004 & $\begin{array}{l}15^{\circ} 17^{\prime} 57^{\prime \prime} \mathrm{S} ; \\
51^{\circ} 10^{\prime} 7^{\prime \prime} \mathrm{W}\end{array}$ & ISN986 & $\begin{array}{l}\text { Nogueira, I.S., } \\
\text { Martins, L. L., da } \\
\text { Silva, W.J., } \\
\text { Rodrigues, J. }\end{array}$ & Phytoplankton \\
\hline UFG29909 & $\begin{array}{l}\text { Brazil, State of Goiás, } \\
\text { Britânia, Lago dos Tigres, } \\
\text { outfall of Luanda Stream, } \\
\text { Santo Antônio Farm, Site } 3\end{array}$ & 09/09/2004 & $\begin{array}{l}15^{\circ} 16^{\prime} 47^{\prime \prime} \mathrm{S} ; \\
51^{\circ} 9^{\prime} 44^{\prime \prime} \mathrm{W}\end{array}$ & ISN987 & $\begin{array}{l}\text { Nogueira, I.S., } \\
\text { Martins, L. L., da } \\
\text { Silva, W.J., } \\
\text { Rodrigues, J. }\end{array}$ & Phytoplankton \\
\hline UFG29910 & $\begin{array}{l}\text { Brazil, State of Goiás, } \\
\text { Britânia, Lago dos Tigres, } \\
\text { Santo Antônio Farm, Site } 4\end{array}$ & 09/09/2004 & $\begin{array}{l}15^{\circ} 16^{\prime} 17^{\prime \prime} \mathrm{S} ; \\
51^{\circ} 9^{\prime} 10^{\prime \prime} \mathrm{W}\end{array}$ & ISN988 & $\begin{array}{l}\text { Nogueira, I.S., } \\
\text { Martins, L. L., da } \\
\text { Silva, W.J., } \\
\text { Rodrigues, J. }\end{array}$ & Phytoplankton \\
\hline UFG29911 & $\begin{array}{l}\text { Brazil, State of Goiás, } \\
\text { Britânia, Lago dos Tigres, } \\
\text { outfall of Arco-Íris Stream, } \\
\text { Site } 5\end{array}$ & 09/09/2004 & $\begin{array}{l}15^{\circ} 15^{\prime} 46^{\prime \prime} \mathrm{S} ; \\
51^{\circ} 8^{\prime} 41^{\prime \prime} \mathrm{W}\end{array}$ & ISN989 & $\begin{array}{l}\text { Nogueira, I.S., } \\
\text { Martins, L. L., da } \\
\text { Silva, W.J., } \\
\text { Rodrigues, J. }\end{array}$ & Phytoplankton \\
\hline UFG29912 & $\begin{array}{l}\text { Brazil, State of Goiás, } \\
\text { Britânia, Lago dos Tigres, } \\
\text { harbor ferry, Site } 6\end{array}$ & 09/09/2004 & $\begin{array}{l}15^{\circ} 15^{\prime} 12^{\prime \prime} \mathrm{S} ; \\
51^{\circ} 8^{\prime} 33^{\prime \prime} \mathrm{W}\end{array}$ & ISN990 & $\begin{array}{l}\text { Nogueira, I.S., } \\
\text { Martins, L. L., da } \\
\text { Silva, W.J., } \\
\text { Rodrigues, J. }\end{array}$ & Phytoplankton \\
\hline UFG29913 & $\begin{array}{l}\text { Brazil, State of Goiás, } \\
\text { Britânia, Lago dos Tigres, } \\
\text { Lago dos Tigres Farm, Site } 7\end{array}$ & 09/09/2004 & $\begin{array}{l}15^{\circ} 14^{\prime} 17^{\prime \prime} \mathrm{S} ; \\
51^{\circ} 8^{\prime} 59^{\prime \prime} \mathrm{W}\end{array}$ & ISN991 & $\begin{array}{l}\text { Nogueira, I.S., } \\
\text { Martins, L. L., da } \\
\text { Silva, W.J., } \\
\text { Rodrigues, J. }\end{array}$ & Phytoplankton \\
\hline
\end{tabular}


Table 2. Continued.

\begin{tabular}{|c|c|c|c|c|c|c|}
\hline $\begin{array}{l}\text { Herbarium } \\
\text { number }\end{array}$ & Locality & Date & Coordinates & $\begin{array}{l}\text { Collector } \\
\text { number }\end{array}$ & Collectors & Habitat \\
\hline UFG29914 & $\begin{array}{l}\text { Brazil, State of Goiás, } \\
\text { Britânia, Lago dos Tigres, city } \\
\text { in front of the Christ, Site } 8\end{array}$ & 09/09/2004 & $\begin{array}{l}15^{\circ} 14^{\prime} 9^{\prime \prime} \mathrm{S} \\
51^{\circ} 9^{\prime} 27^{\prime \prime} \mathrm{W}\end{array}$ & ISN992 & $\begin{array}{l}\text { Nogueira, I.S., } \\
\text { Martins, L. L., da } \\
\text { Silva, W.J., } \\
\text { Rodrigues, J. }\end{array}$ & Phytoplankton \\
\hline UFG29915 & $\begin{array}{l}\text { Brazil, State of Goiás, } \\
\text { Britânia, Lago dos Tigres, } \\
\text { downstream of the "lake", } \\
\text { Lago dos Tigres Farm, Site } 9\end{array}$ & $10 / 09 / 2004$ & $\begin{array}{l}15^{\circ} 13^{\prime} 43^{\prime \prime} \mathrm{S} \\
51^{\circ} 9^{\prime} 14^{\prime \prime} \mathrm{W}\end{array}$ & ISN993 & $\begin{array}{l}\text { Nogueira, I.S., } \\
\text { Martins, L. L., da } \\
\text { Silva, W.J., } \\
\text { Rodrigues, J. }\end{array}$ & Phytoplankton \\
\hline UFG29916 & $\begin{array}{l}\text { Brazil, State of Goiás, } \\
\text { Britânia, Lago dos Tigres, } \\
\text { outfall of the "lake" in } \\
\text { Vermelho River, Site } 10\end{array}$ & $10 / 09 / 2004$ & $\begin{array}{l}15^{\circ} 13^{\prime} 18^{\prime \prime} \mathrm{S} \\
51^{\circ} 10^{\prime} 6^{\prime \prime} \mathrm{W}\end{array}$ & ISN994 & $\begin{array}{l}\text { Nogueira, I.S., } \\
\text { Martins, L. L., da } \\
\text { Silva, W.J., } \\
\text { Rodrigues, J. }\end{array}$ & Phytoplankton \\
\hline UFG29917 & $\begin{array}{l}\text { Brazil, State of Goiás, } \\
\text { Britânia, Lago dos Tigres, } \\
\text { new channel of the Vermelho } \\
\text { River, Site } 11\end{array}$ & $10 / 09 / 2004$ & $\begin{array}{l}15^{\circ} 11^{\prime} 47^{\prime \prime} \mathrm{S} \\
51^{\circ} 9^{\prime} 57^{\prime \prime} \mathrm{W}\end{array}$ & ISN995 & $\begin{array}{l}\text { Nogueira, I.S., } \\
\text { Martins, L. L., da } \\
\text { Silva, W.J., } \\
\text { Rodrigues, J. }\end{array}$ & Phytoplankton \\
\hline UFG29918 & $\begin{array}{l}\text { Brazil, State of Goiás, } \\
\text { Britânia, Água Limpa River, } \\
\text { Água Limpa Farm, Site } 1\end{array}$ & $16 / 10 / 2004$ & $\begin{array}{l}15^{\circ} 18^{\prime} 58^{\prime \prime} \mathrm{S} \\
51^{\circ} 9^{\prime} 56^{\prime \prime} \mathrm{W}\end{array}$ & ISN1023 & $\begin{array}{l}\text { Nogueira, I.S., } \\
\text { Nabout, J.C., } \\
\text { Oliveira, J.E., } \\
\text { Valente, C. }\end{array}$ & Phytoplankton \\
\hline UFG29919 & $\begin{array}{l}\text { Brazil, State of Goiás, } \\
\text { Britânia, Lago dos Tigres, } \\
\text { Perdidas Island, Site } 2\end{array}$ & $16 / 10 / 2004$ & $\begin{array}{l}15^{\circ} 17^{\prime} 57^{\prime \prime} \mathrm{S} \\
51^{\circ} 10^{\prime} 7^{\prime \prime} \mathrm{W}\end{array}$ & ISN1024 & $\begin{array}{l}\text { Nogueira, I.S., } \\
\text { Nabout, J.C., } \\
\text { Oliveira, J.E., } \\
\text { Valente, C. }\end{array}$ & Phytoplankton \\
\hline UFG29920 & $\begin{array}{l}\text { Brazil, State of Goiás, } \\
\text { Britânia, Lago dos Tigres, } \\
\text { outfall of Luanda Stream, } \\
\text { Santo Antônio Farm, Site } 3\end{array}$ & $16 / 10 / 2004$ & $\begin{array}{l}15^{\circ} 16^{\prime} 47^{\prime \prime} \mathrm{S} \\
51^{\circ} 9^{\prime} 44^{\prime \prime} \mathrm{W}\end{array}$ & ISN1025 & $\begin{array}{l}\text { Nogueira, I.S., } \\
\text { Nabout, J.C., } \\
\text { Oliveira, J.E., } \\
\text { Valente, C. }\end{array}$ & Phytoplankton \\
\hline UFG29921 & $\begin{array}{l}\text { Brazil, State of Goiás, } \\
\text { Britânia, Lago dos Tigres, } \\
\text { Santo Antônio Farm, Site } 4\end{array}$ & $16 / 10 / 2004$ & $\begin{array}{l}15^{\circ} 16^{\prime} 17^{\prime \prime} \mathrm{S} \\
51^{\circ} 9^{\prime} 10^{\prime \prime} \mathrm{W}\end{array}$ & ISN1026 & $\begin{array}{l}\text { Nogueira, I.S., } \\
\text { Nabout, J.C., } \\
\text { Oliveira, J.E., } \\
\text { Valente, C. }\end{array}$ & Phytoplankton \\
\hline UFG29922 & $\begin{array}{l}\text { Brazil, State of Goiás, } \\
\text { Britânia, Lago dos Tigres, } \\
\text { outfall of Arco-Íris Stream, } \\
\text { Site } 5\end{array}$ & $16 / 10 / 2004$ & $\begin{array}{l}15^{\circ} 15^{\prime} 46^{\prime \prime} \mathrm{S} \\
51^{\circ} 8^{\prime} 41^{\prime \prime} \mathrm{W}\end{array}$ & ISN1027 & $\begin{array}{l}\text { Nogueira, I.S., } \\
\text { Nabout, J.C., } \\
\text { Oliveira, J.E., } \\
\text { Valente, C. }\end{array}$ & Phytoplankton \\
\hline UFG29923 & $\begin{array}{l}\text { Brazil, State of Goiás, Britânia, } \\
\text { Lago dos Tigres, harbor ferry, } \\
\text { Site } 6\end{array}$ & $16 / 10 / 2004$ & $\begin{array}{l}15^{\circ} 15^{\prime} 12^{\prime \prime} \mathrm{S} \\
51^{\circ} 8^{\prime} 33^{\prime \prime} \mathrm{W}\end{array}$ & ISN1028 & $\begin{array}{l}\text { Nogueira, I.S., } \\
\text { Nabout, J.C., } \\
\text { Oliveira, J.E., } \\
\text { Valente, C. }\end{array}$ & Phytoplankton \\
\hline UFG29924 & $\begin{array}{l}\text { Brazil, State of Goiás, } \\
\text { Britânia, Lago dos Tigres, } \\
\text { Lago dos Tigres Farm, Site } 7\end{array}$ & $16 / 10 / 2004$ & $\begin{array}{l}15^{\circ} 14^{\prime} 17^{\prime \prime} \mathrm{S} \\
51^{\circ} 8^{\prime} 59^{\prime \prime} \mathrm{W}\end{array}$ & ISN1029 & $\begin{array}{l}\text { Nogueira, I.S., } \\
\text { Nabout, J.C., } \\
\text { Oliveira, J.E., } \\
\text { Valente, C. }\end{array}$ & Phytoplankton \\
\hline UFG29925 & $\begin{array}{l}\text { Brazil, State of Goiás, } \\
\text { Britânia, Lago dos Tigres, city } \\
\text { in front of the Christ, Site } 8\end{array}$ & $16 / 10 / 2004$ & $\begin{array}{l}15^{\circ} 14^{\prime} 9^{\prime \prime} \mathrm{S} \\
51^{\circ} 9^{\prime} 27^{\prime \prime} \mathrm{W}\end{array}$ & ISN1030 & $\begin{array}{l}\text { Nogueira, I.S., } \\
\text { Nabout, J.C., } \\
\text { Oliveira, J.E., } \\
\text { Valente, C. }\end{array}$ & Phytoplankton \\
\hline UFG29926 & $\begin{array}{l}\text { Brazil, State of Goiás, } \\
\text { Britânia, Lago dos Tigres, } \\
\text { downstream of the "lake", } \\
\text { Lago dos Tigres Farm, Site } 9\end{array}$ & $17 / 10 / 2004$ & $\begin{array}{l}15^{\circ} 13^{\prime} 43^{\prime \prime} \mathrm{S} \\
51^{\circ} 9^{\prime} 14^{\prime \prime} \mathrm{W}\end{array}$ & ISN1031 & $\begin{array}{l}\text { Nogueira, I.S., } \\
\text { Nabout, J.C., } \\
\text { Oliveira, J.E., } \\
\text { Valente, C. }\end{array}$ & Phytoplankton \\
\hline UFG29927 & $\begin{array}{l}\text { Brazil, State of Goiás, } \\
\text { Britânia, Lago dos Tigres, } \\
\text { outfall of the "lake" in } \\
\text { Vermelho River, Site } 10\end{array}$ & $17 / 10 / 2004$ & $\begin{array}{l}15^{\circ} 13^{\prime} 18^{\prime \prime} \mathrm{S} ; \\
51^{\circ} 10^{\prime} 6^{\prime \prime} \mathrm{W}\end{array}$ & ISN1032 & $\begin{array}{l}\text { Nogueira, I.S., } \\
\text { Nabout, J.C., } \\
\text { Oliveira, J.E., } \\
\text { Valente, C. }\end{array}$ & Phytoplankton \\
\hline
\end{tabular}


Table 2. Continued.

\begin{tabular}{|c|c|c|c|c|c|c|}
\hline $\begin{array}{l}\text { Herbarium } \\
\text { number }\end{array}$ & Locality & Date & Coordinates & $\begin{array}{c}\text { Collector } \\
\text { number }\end{array}$ & Collectors & Habitat \\
\hline UFG29928 & $\begin{array}{l}\text { Brazil, State of Goiás, } \\
\text { Britânia, Lago dos Tigres, } \\
\text { new channel of the Vermelho } \\
\text { River, Site } 11\end{array}$ & $17 / 10 / 2004$ & $\begin{array}{l}15^{\circ} 11^{\prime} 47^{\prime \prime} \mathrm{S} ; \\
51^{\circ} 9^{\prime} 57^{\prime \prime} \mathrm{W}\end{array}$ & ISN1033 & $\begin{array}{l}\text { Nogueira, I.S., } \\
\text { Nabout, J.C., } \\
\text { Oliveira, J.E., } \\
\text { Valente, C. }\end{array}$ & Phytoplankton \\
\hline UFG29929 & $\begin{array}{l}\text { Brazil, State of Goiás, } \\
\text { Britânia, Água Limpa River, } \\
\text { Água Limpa Farm, Site } 1\end{array}$ & $05 / 11 / 2004$ & $\begin{array}{l}15^{\circ} 18^{\prime} 58^{\prime \prime} \mathrm{S} \\
51^{\circ} 9^{\prime} 56^{\prime \prime} \mathrm{W}\end{array}$ & ISN1115 & $\begin{array}{l}\text { Nogueira, I.S., } \\
\text { Martins, L. L., da } \\
\text { Silva, W.J., Uto, F.N. }\end{array}$ & Phytoplankton \\
\hline UFG29930 & $\begin{array}{l}\text { Brazil, State of Goiás, } \\
\text { Britânia, Lago dos Tigres, } \\
\text { Perdidas Island, Site } 2\end{array}$ & $05 / 11 / 2004$ & $\begin{array}{l}15^{\circ} 17^{\prime} 57^{\prime \prime} \mathrm{S} \\
51^{\circ} 10^{\prime} 7^{\prime \prime} \mathrm{W}\end{array}$ & ISN1116 & $\begin{array}{l}\text { Nogueira, I.S., } \\
\text { Martins, L. L., da } \\
\text { Silva, W.J., Uto, F.N. }\end{array}$ & Phytoplankton \\
\hline UFG29931 & $\begin{array}{l}\text { Brazil, State of Goiás, Britânia, } \\
\text { Lago dos Tigres, outfall of } \\
\text { Luanda Stream, Santo Antônio } \\
\text { Farm, Site } 3\end{array}$ & $05 / 11 / 2004$ & $\begin{array}{l}15^{\circ} 16^{\prime} 47^{\prime \prime} \mathrm{S} ; \\
51^{\circ} 9^{\prime} 44^{\prime \prime} \mathrm{W}\end{array}$ & ISN1117 & $\begin{array}{l}\text { Nogueira, I.S., } \\
\text { Martins, L. L., da } \\
\text { Silva, W.J., Uto, F.N. }\end{array}$ & Phytoplankton \\
\hline UFG29932 & $\begin{array}{l}\text { Brazil, State of Goiás, } \\
\text { Britânia, Lago dos Tigres, } \\
\text { Santo Antônio Farm, Site } 4\end{array}$ & $05 / 11 / 2004$ & $\begin{array}{l}15^{\circ} 16^{\prime} 17^{\prime \prime} \mathrm{S} ; \\
51^{\circ} 9^{\prime} 10^{\prime \prime} \mathrm{W}\end{array}$ & ISN1118 & $\begin{array}{l}\text { Nogueira, I.S., } \\
\text { Martins, L. L., da } \\
\text { Silva, W.J., Uto, F.N. }\end{array}$ & Phytoplankton \\
\hline UFG29933 & $\begin{array}{l}\text { Brazil, State of Goiás, } \\
\text { Britânia, Lago dos Tigres, } \\
\text { outfall of Arco-Íris Stream, } \\
\text { Site } 5\end{array}$ & $05 / 11 / 2004$ & $\begin{array}{l}15^{\circ} 15^{\prime} 46^{\prime \prime} \mathrm{S} ; \\
51^{\circ} 8^{\prime} 41^{\prime \prime} \mathrm{W}\end{array}$ & ISN1119 & $\begin{array}{l}\text { Nogueira, I.S., } \\
\text { Martins, L. L., da } \\
\text { Silva, W.J., Uto, F.N. }\end{array}$ & Phytoplankton \\
\hline UFG29934 & $\begin{array}{l}\text { Brazil, State of Goiás, } \\
\text { Britânia, Lago dos Tigres, } \\
\text { harbor ferry, Site } 6\end{array}$ & $05 / 11 / 2004$ & $\begin{array}{l}15^{\circ} 15^{\prime} 12^{\prime \prime} \mathrm{S} ; \\
51^{\circ} 8^{\prime} 33^{\prime \prime} \mathrm{W}\end{array}$ & ISN1120 & $\begin{array}{l}\text { Nogueira, I.S., } \\
\text { Martins, L. L., da } \\
\text { Silva, W.J., Uto, F.N. }\end{array}$ & Phytoplankton \\
\hline UFG29935 & $\begin{array}{l}\text { Brazil, State of Goiás, } \\
\text { Britânia, Lago dos Tigres, } \\
\text { Lago dos Tigres Farm, Site } 7\end{array}$ & $05 / 11 / 2004$ & $\begin{array}{l}15^{\circ} 14^{\prime} 17^{\prime \prime} \mathrm{S} ; \\
51^{\circ} 8^{\prime} 59^{\prime \prime} \mathrm{W}\end{array}$ & ISN1121 & $\begin{array}{l}\text { Nogueira, I.S., } \\
\text { Martins, L. L., da } \\
\text { Silva, W.J., Uto, F.N. }\end{array}$ & Phytoplankton \\
\hline UFG29936 & $\begin{array}{l}\text { Brazil, State of Goiás, } \\
\text { Britânia, Lago dos Tigres, city } \\
\text { in front of the Christ, Site } 8\end{array}$ & $05 / 11 / 2004$ & $\begin{array}{l}15^{\circ} 14^{\prime} 9^{\prime \prime} \mathrm{S} \\
51^{\circ} 9^{\prime} 27^{\prime \prime} \mathrm{W}\end{array}$ & ISN1122 & $\begin{array}{l}\text { Nogueira, I.S., } \\
\text { Martins, L. L., da } \\
\text { Silva, W.J., Uto, F.N. }\end{array}$ & Phytoplankton \\
\hline UFG29937 & $\begin{array}{l}\text { Brazil, State of Goiás, } \\
\text { Britânia, Lago dos Tigres, } \\
\text { downstream of the "lake", } \\
\text { Lago dos Tigres Farm, Site } 9\end{array}$ & $06 / 11 / 2004$ & $\begin{array}{l}15^{\circ} 13^{\prime} 43^{\prime \prime} \mathrm{S} ; \\
51^{\circ} 9^{\prime} 14^{\prime \prime} \mathrm{W}\end{array}$ & ISN1123 & $\begin{array}{l}\text { Nogueira, I.S., } \\
\text { Martins, L. L., da } \\
\text { Silva, W.J., Uto, F.N. }\end{array}$ & Phytoplankton \\
\hline UFG29938 & $\begin{array}{l}\text { Brazil, State of Goiás, } \\
\text { Britânia, Lago dos Tigres, } \\
\text { outfall of the "lake" in } \\
\text { Vermelho River, Site } 10\end{array}$ & $06 / 11 / 2004$ & $\begin{array}{l}15^{\circ} 13^{\prime} 18^{\prime \prime} \mathrm{S} ; \\
51^{\circ} 10^{\prime} 6^{\prime \prime} \mathrm{W}\end{array}$ & ISN1124 & $\begin{array}{l}\text { Nogueira, I.S., } \\
\text { Martins, L. L., da } \\
\text { Silva, W.J., Uto, F.N. }\end{array}$ & Phytoplankton \\
\hline UFG29939 & $\begin{array}{l}\text { Brazil, State of Goiás, } \\
\text { Britânia, Lago dos Tigres, } \\
\text { new channel of the Vermelho } \\
\text { River, Site } 11\end{array}$ & $06 / 11 / 2004$ & $\begin{array}{l}15^{\circ} 11^{\prime} 47^{\prime \prime} \mathrm{S} ; \\
51^{\circ} 9^{\prime} 57^{\prime \prime} \mathrm{W}\end{array}$ & ISN1125 & $\begin{array}{l}\text { Nogueira, I.S., } \\
\text { Martins, L. L., da } \\
\text { Silva, W.J., Uto, F.N. }\end{array}$ & Phytoplankton \\
\hline UFG29940 & $\begin{array}{l}\text { Brazil, State of Goiás, } \\
\text { Britânia, Água Limpa River, } \\
\text { Água Limpa Farm, Site } 1\end{array}$ & $13 / 07 / 2004$ & $\begin{array}{l}15^{\circ} 18^{\prime} 58^{\prime \prime} \mathrm{S} \\
51^{\circ} 9^{\prime} 56^{\prime \prime} \mathrm{W}\end{array}$ & ISN934 & $\begin{array}{l}\text { Nogueira, I.S., da } \\
\text { Silva, W.J., Nabout, J. } \\
\text { C., Oliveira, J.E., } \\
\text { Jorge, V.A }\end{array}$ & Periphyton \\
\hline UFG29941 & $\begin{array}{l}\text { Brazil, State of Goiás, } \\
\text { Britânia, Lago dos Tigres, } \\
\text { Perdidas Island, Site } 2\end{array}$ & $13 / 07 / 2004$ & $\begin{array}{l}15^{\circ} 17^{\prime} 57^{\prime \prime} \mathrm{S} ; \\
51^{\circ} 10^{\prime} 7^{\prime \prime} \mathrm{W}\end{array}$ & ISN935 & $\begin{array}{l}\text { Nogueira, I.S., da } \\
\text { Silva, W.J., Nabout, J. } \\
\text { C., Oliveira, J.E., } \\
\text { Jorge, V.A }\end{array}$ & Periphyton \\
\hline UFG29942 & $\begin{array}{l}\text { Brazil, State of Goiás, } \\
\text { Britânia, Lago dos Tigres, } \\
\text { Perdidas Island, Site } 2\end{array}$ & $13 / 07 / 2004$ & $\begin{array}{l}15^{\circ} 17^{\prime} 57^{\prime \prime} \mathrm{S} ; \\
51^{\circ} 10^{\prime} 7^{\prime \prime} \mathrm{W}\end{array}$ & ISN936 & $\begin{array}{l}\text { Nogueira, I.S., da } \\
\text { Silva, W.J., Nabout, J. } \\
\text { C., Oliveira, J.E., } \\
\text { Jorge, V.A }\end{array}$ & Periphyton \\
\hline
\end{tabular}


Table 2. Continued.

\begin{tabular}{|c|c|c|c|c|c|c|}
\hline $\begin{array}{l}\text { Herbarium } \\
\text { number }\end{array}$ & Locality & Date & Coordinates & $\begin{array}{l}\text { Collector } \\
\text { number }\end{array}$ & Collectors & Habitat \\
\hline UFG29943 & $\begin{array}{l}\text { Brazil, State of Goiás, } \\
\text { Britânia, Água Limpa River, } \\
\text { Água Limpa Farm, Site } 1\end{array}$ & $13 / 07 / 2004$ & $\begin{array}{l}15^{\circ} 18^{\prime} 58^{\prime \prime} \mathrm{S} \\
51^{\circ} 9^{\prime} 56^{\prime \prime} \mathrm{W}\end{array}$ & ISN937 & $\begin{array}{l}\text { Nogueira, I.S., da } \\
\text { Silva, W.J., Nabout, J. } \\
\text { C., Oliveira, J.E., } \\
\text { Jorge, V.A }\end{array}$ & Periphyton \\
\hline UFG29944 & $\begin{array}{l}\text { Brazil, State of Goiás, } \\
\text { Britânia, Lago dos Tigres, } \\
\text { Perdidas Island, Site } 2\end{array}$ & $13 / 07 / 2004$ & $\begin{array}{l}15^{\circ} 17^{\prime} 57^{\prime \prime} \mathrm{S} \\
51^{\circ} 10^{\prime} 7^{\prime \prime} \mathrm{W}\end{array}$ & ISN938 & $\begin{array}{l}\text { Nogueira, I.S., da } \\
\text { Silva, W.J., Nabout, J. } \\
\text { C., Oliveira, J.E., } \\
\text { Jorge, V.A }\end{array}$ & Periphyton \\
\hline UFG29946 & $\begin{array}{l}\text { Brazil, State of Goiás, } \\
\text { Britânia, Água Limpa River, } \\
\text { Água Limpa Farm, Site } 1\end{array}$ & $19 / 08 / 2004$ & $\begin{array}{l}15^{\circ} 18^{\prime} 58^{\prime \prime} \mathrm{S} \\
51^{\circ} 9^{\prime} 56^{\prime \prime} \mathrm{W}\end{array}$ & ISN973 & $\begin{array}{l}\text { Nogueira, I.S., } \\
\text { Martins, L.L., Uto, F. } \\
\text { N., Nabout, J.C. }\end{array}$ & Periphyton \\
\hline UFG29947 & $\begin{array}{l}\text { Brazil, State of Goiás, } \\
\text { Britânia, Água Limpa River, } \\
\text { Água Limpa Farm, Site } 1\end{array}$ & $19 / 08 / 2004$ & $\begin{array}{l}15^{\circ} 18^{\prime} 58^{\prime \prime} \mathrm{S} \\
51^{\circ} 9^{\prime} 56^{\prime \prime} \mathrm{W}\end{array}$ & ISN974 & $\begin{array}{l}\text { Nogueira, I.S., } \\
\text { Martins, L.L., Uto, F. } \\
\text { N., Nabout, J.C. }\end{array}$ & Periphyton \\
\hline UFG29948 & $\begin{array}{l}\text { Brazil, State of Goiás, } \\
\text { Britânia, Água Limpa River, } \\
\text { Água Limpa Farm, Site } 1\end{array}$ & $19 / 08 / 2004$ & $\begin{array}{l}15^{\circ} 18^{\prime} 58^{\prime \prime} \mathrm{S} \\
51^{\circ} 9^{\prime} 56^{\prime \prime} \mathrm{W}\end{array}$ & ISN975 & $\begin{array}{l}\text { Nogueira, I.S., } \\
\text { Martins, L.L., Uto, F. } \\
\text { N., Nabout, J.C. }\end{array}$ & Periphyton \\
\hline UFG29949 & $\begin{array}{l}\text { Brazil, State of Goiás, } \\
\text { Britânia, Água Limpa River, } \\
\text { Água Limpa Farm, Site } 1\end{array}$ & $19 / 08 / 2004$ & $\begin{array}{l}15^{\circ} 18^{\prime} 58^{\prime \prime} \mathrm{S} \\
51^{\circ} 9^{\prime} 56^{\prime \prime} \mathrm{W}\end{array}$ & ISN976 & $\begin{array}{l}\text { Nogueira, I.S., } \\
\text { Martins, L.L., Uto, F. } \\
\text { N., Nabout, J.C. }\end{array}$ & Periphyton \\
\hline UFG29950 & $\begin{array}{l}\text { Brazil, State of Goiás, } \\
\text { Britânia, Lago dos Tigres, } \\
\text { Perdidas Island, Site } 2\end{array}$ & $19 / 08 / 2004$ & $\begin{array}{l}15^{\circ} 17^{\prime} 57^{\prime \prime} \mathrm{S} \\
51^{\circ} 10^{\prime} 7^{\prime \prime} \mathrm{W}\end{array}$ & ISN977 & $\begin{array}{l}\text { Nogueira, I.S., } \\
\text { Martins, L.L., Uto, F. } \\
\text { N., Nabout, J.C. }\end{array}$ & Periphyton \\
\hline UFG29951 & $\begin{array}{l}\text { Brazil, State of Goiás, } \\
\text { Britânia, Lago dos Tigres, } \\
\text { Perdidas Island, Site } 2\end{array}$ & $19 / 08 / 2004$ & $\begin{array}{l}15^{\circ} 17^{\prime} 57^{\prime \prime} \mathrm{S} ; \\
51^{\circ} 10^{\prime} 7^{\prime \prime} \mathrm{W}\end{array}$ & ISN978 & $\begin{array}{l}\text { Nogueira, I.S., } \\
\text { Martins, L.L., Uto, F. } \\
\text { N., Nabout, J.C. }\end{array}$ & Periphyton \\
\hline UFG29952 & $\begin{array}{l}\text { Brazil, State of Goiás, } \\
\text { Britânia, Água Limpa River, } \\
\text { Água Limpa Farm, Site } 1\end{array}$ & 09/09/2004 & $\begin{array}{l}15^{\circ} 18^{\prime} 58^{\prime \prime} \mathrm{S} \\
51^{\circ} 9^{\prime} 56^{\prime \prime} \mathrm{W}\end{array}$ & ISN1013 & $\begin{array}{l}\text { Nogueira, I.S., } \\
\text { Martins, L.L., da } \\
\text { Silva, W.J., } \\
\text { Rodrigues, J. }\end{array}$ & Periphyton \\
\hline UFG29953 & $\begin{array}{l}\text { Brazil, State of Goiás, } \\
\text { Britânia, Água Limpa River, } \\
\text { Água Limpa Farm, Site } 1\end{array}$ & 09/09/2004 & $\begin{array}{l}15^{\circ} 18^{\prime} 58^{\prime \prime} \mathrm{S} \\
51^{\circ} 9^{\prime} 56^{\prime \prime} \mathrm{W}\end{array}$ & ISN1014 & $\begin{array}{l}\text { Nogueira, I.S., } \\
\text { Martins, L.L., da } \\
\text { Silva, W.J., } \\
\text { Rodrigues, J. }\end{array}$ & Periphyton \\
\hline UFG29954 & $\begin{array}{l}\text { Brazil, State of Goiás, } \\
\text { Britânia, Água Limpa River, } \\
\text { Água Limpa Farm, Site } 1\end{array}$ & 09/09/2004 & $\begin{array}{l}15^{\circ} 18^{\prime} 58^{\prime \prime} \mathrm{S} \\
51^{\circ} 9^{\prime} 56^{\prime \prime} \mathrm{W}\end{array}$ & ISN1015 & $\begin{array}{l}\text { Nogueira, I.S., } \\
\text { Martins, L.L., da } \\
\text { Silva, W.J., } \\
\text { Rodrigues, J. }\end{array}$ & Periphyton \\
\hline UFG29955 & $\begin{array}{l}\text { Brazil, State of Goiás, } \\
\text { Britânia, Lago dos Tigres, } \\
\text { Perdidas Island, Site } 2\end{array}$ & 09/09/2004 & $\begin{array}{l}15^{\circ} 17^{\prime} 57^{\prime \prime} \mathrm{S} \\
51^{\circ} 10^{\prime} 7^{\prime \prime} \mathrm{W}\end{array}$ & ISN1016 & $\begin{array}{l}\text { Nogueira, I.S., } \\
\text { Martins, L.L., da } \\
\text { Silva, W.J., } \\
\text { Rodrigues, J. }\end{array}$ & Periphyton \\
\hline UFG29956 & $\begin{array}{l}\text { Brazil, State of Goiás, } \\
\text { Britânia, Lago dos Tigres, } \\
\text { Perdidas Island, Site } 2\end{array}$ & 09/09/2004 & $\begin{array}{l}15^{\circ} 17^{\prime} 57^{\prime \prime} \mathrm{S} \\
51^{\circ} 10^{\prime} 7^{\prime \prime} \mathrm{W}\end{array}$ & ISN1017 & $\begin{array}{l}\text { Nogueira, I.S., } \\
\text { Martins, L.L., da } \\
\text { Silva, W.J., } \\
\text { Rodrigues, J. }\end{array}$ & Periphyton \\
\hline UFG29957 & $\begin{array}{l}\text { Brazil, State of Goiás, } \\
\text { Britânia, Água Limpa River, } \\
\text { Água Limpa Farm, Site } 1\end{array}$ & $16 / 10 / 2004$ & $\begin{array}{l}15^{\circ} 18^{\prime} 58^{\prime \prime} \mathrm{S} \\
51^{\circ} 9^{\prime} 56^{\prime \prime} \mathrm{W}\end{array}$ & ISN1126 & $\begin{array}{l}\text { Nogueira, I.S., } \\
\text { Nabout, J.C., } \\
\text { Oliveira, J.E., } \\
\text { Valente, C. }\end{array}$ & Periphyton \\
\hline UFG29958 & $\begin{array}{l}\text { Brazil, State of Goiás, } \\
\text { Britânia, Água Limpa River, } \\
\text { Água Limpa Farm, Site } 1\end{array}$ & $16 / 10 / 2004$ & $\begin{array}{l}15^{\circ} 18^{\prime} 58^{\prime \prime} \mathrm{S} \\
51^{\circ} 9^{\prime} 56^{\prime \prime} \mathrm{W}\end{array}$ & ISN1127 & $\begin{array}{l}\text { Nogueira, I.S., } \\
\text { Nabout, J.C., } \\
\text { Oliveira, J.E., } \\
\text { Valente, C. }\end{array}$ & Periphyton \\
\hline
\end{tabular}


Table 2. Continued.

\begin{tabular}{|c|c|c|c|c|c|c|}
\hline $\begin{array}{l}\text { Herbarium } \\
\text { number }\end{array}$ & Locality & Date & Coordinates & $\begin{array}{l}\text { Collector } \\
\text { number }\end{array}$ & Collectors & Habitat \\
\hline UFG29959 & $\begin{array}{l}\text { Brazil, State of Goiás, } \\
\text { Britânia, Lago dos Tigres, } \\
\text { Perdidas Island, Site } 2\end{array}$ & $16 / 10 / 2004$ & $\begin{array}{l}15^{\circ} 17^{\prime} 57^{\prime \prime} \mathrm{S} ; \\
51^{\circ} 10^{\prime} 7^{\prime \prime} \mathrm{W}\end{array}$ & ISN1128 & $\begin{array}{l}\text { Nogueira, I.S., } \\
\text { Nabout, J.C., } \\
\text { Oliveira, J.E., } \\
\text { Valente, C. }\end{array}$ & Periphyton \\
\hline UFG29960 & $\begin{array}{l}\text { Brazil, State of Goiás, } \\
\text { Britânia, Lago dos Tigres, } \\
\text { Perdidas Island, Site } 2\end{array}$ & $16 / 10 / 2004$ & $\begin{array}{l}15^{\circ} 17^{\prime} 57^{\prime \prime} \mathrm{S} ; \\
51^{\circ} 10^{\prime} 7^{\prime \prime} \mathrm{W}\end{array}$ & ISN1129 & $\begin{array}{l}\text { Nogueira, I.S., } \\
\text { Nabout, J.C., } \\
\text { Oliveira, J.E., } \\
\text { Valente, C. }\end{array}$ & Periphyton \\
\hline UFG29961 & $\begin{array}{l}\text { Brazil, State of Goiás, } \\
\text { Britânia, Lago dos Tigres, } \\
\text { Perdidas Island, Site } 2\end{array}$ & $16 / 10 / 2004$ & $\begin{array}{l}15^{\circ} 17^{\prime} 57^{\prime \prime} \mathrm{S} ; \\
51^{\circ} 10^{\prime} 7^{\prime \prime} \mathrm{W}\end{array}$ & ISN1130 & $\begin{array}{l}\text { Nogueira, I.S., } \\
\text { Nabout, J.C., } \\
\text { Oliveira, J.E., } \\
\text { Valente, C. }\end{array}$ & Periphyton \\
\hline UFG29962 & $\begin{array}{l}\text { Brazil, State of Goiás, } \\
\text { Britânia, Lago dos Tigres, } \\
\text { Perdidas Island, Site } 2\end{array}$ & $16 / 10 / 2004$ & $\begin{array}{l}15^{\circ} 17^{\prime} 57^{\prime \prime} \mathrm{S} ; \\
51^{\circ} 10^{\prime} 7^{\prime \prime} \mathrm{W}\end{array}$ & ISN1131 & $\begin{array}{l}\text { Nogueira, I.S., } \\
\text { Nabout, J.C., } \\
\text { Oliveira, J.E., } \\
\text { Valente, C. }\end{array}$ & Periphyton \\
\hline UFG29963 & $\begin{array}{l}\text { Brazil, State of Goiás, } \\
\text { Britânia, Água Limpa River, } \\
\text { Água Limpa Farm, Site } 1\end{array}$ & $05 / 11 / 2004$ & $\begin{array}{l}15^{\circ} 18^{\prime} 58^{\prime \prime} \mathrm{S} ; \\
51^{\circ} 9^{\prime} 56^{\prime \prime} \mathrm{W}\end{array}$ & ISN1132 & $\begin{array}{l}\text { Nogueira, I.S., } \\
\text { Martins, L.L., da } \\
\text { Silva, W.J., Uto, F.N. }\end{array}$ & Periphyton \\
\hline UFG29964 & $\begin{array}{l}\text { Brazil, State of Goiás, } \\
\text { Britânia, Água Limpa River, } \\
\text { Água Limpa Farm, Site } 1\end{array}$ & $05 / 11 / 2004$ & $\begin{array}{l}15^{\circ} 18^{\prime} 58^{\prime \prime} \mathrm{S} \\
51^{\circ} 9^{\prime} 56^{\prime \prime} \mathrm{W}\end{array}$ & ISN1133 & $\begin{array}{l}\text { Nogueira, I.S., } \\
\text { Martins, L.L., da } \\
\text { Silva, W.J., Uto, F.N. }\end{array}$ & Periphyton \\
\hline UFG29965 & $\begin{array}{l}\text { Brazil, State of Goiás, } \\
\text { Britânia, Água Limpa River, } \\
\text { Água Limpa Farm, Site } 1\end{array}$ & $05 / 11 / 2004$ & $\begin{array}{l}15^{\circ} 18^{\prime} 58^{\prime \prime} \mathrm{S} ; \\
51^{\circ} 9^{\prime} 56^{\prime \prime} \mathrm{W}\end{array}$ & ISN1134 & $\begin{array}{l}\text { Nogueira, I.S., } \\
\text { Martins, L.L., da } \\
\text { Silva, W.J., Uto, F.N. }\end{array}$ & Periphyton \\
\hline UFG29966 & $\begin{array}{l}\text { Brazil, State of Goiás, } \\
\text { Britânia, Água Limpa River, } \\
\text { Água Limpa Farm, Site } 1\end{array}$ & $05 / 11 / 2004$ & $\begin{array}{l}15^{\circ} 18^{\prime} 58^{\prime \prime} \mathrm{S} \\
51^{\circ} 9^{\prime} 56^{\prime \prime} \mathrm{W}\end{array}$ & ISN1135 & $\begin{array}{l}\text { Nogueira, I.S., } \\
\text { Martins, L.L., da } \\
\text { Silva, W.J., Uto, F.N. }\end{array}$ & Periphyton \\
\hline UFG29967 & $\begin{array}{l}\text { Brazil, State of Goiás, } \\
\text { Britânia, Lago dos Tigres, } \\
\text { Perdidas Island, Site } 2\end{array}$ & $05 / 11 / 2004$ & $\begin{array}{l}15^{\circ} 17^{\prime} 57^{\prime \prime} \mathrm{S} ; \\
51^{\circ} 10^{\prime} 7^{\prime \prime} \mathrm{W}\end{array}$ & ISN1136 & $\begin{array}{l}\text { Nogueira, I.S., } \\
\text { Martins, L.L., da } \\
\text { Silva, W.J., Uto, F.N. }\end{array}$ & Periphyton \\
\hline UFG29968 & $\begin{array}{l}\text { Brazil, State of Goiás, } \\
\text { Britânia, Lago dos Tigres, } \\
\text { Perdidas Island, Site } 2\end{array}$ & $05 / 11 / 2004$ & $\begin{array}{l}15^{\circ} 17^{\prime} 57^{\prime \prime} \mathrm{S} ; \\
51^{\circ} 10^{\prime} 7^{\prime \prime} \mathrm{W}\end{array}$ & ISN1137 & $\begin{array}{l}\text { Nogueira, I.S., } \\
\text { Martins, L.L., da } \\
\text { Silva, W.J., Uto, F.N. }\end{array}$ & Periphyton \\
\hline UFG29969 & $\begin{array}{l}\text { Brazil, State of Goiás, } \\
\text { Britânia, Lago dos Tigres, } \\
\text { Perdidas Island, Site } 2\end{array}$ & $05 / 11 / 2004$ & $\begin{array}{l}15^{\circ} 17^{\prime} 57^{\prime \prime} \mathrm{S} ; \\
51^{\circ} 10^{\prime} 7^{\prime \prime} \mathrm{W}\end{array}$ & ISN1138 & $\begin{array}{l}\text { Nogueira, I.S., } \\
\text { Martins, L.L., da } \\
\text { Silva, W.J., Uto, F.N. }\end{array}$ & Periphyton \\
\hline UFG29970 & $\begin{array}{l}\text { Brazil, State of Goiás, } \\
\text { Britânia, Lago dos Tigres, } \\
\text { Perdidas Island, Site } 2\end{array}$ & $05 / 11 / 2004$ & $\begin{array}{l}15^{\circ} 17^{\prime} 57^{\prime \prime} \mathrm{S} ; \\
51^{\circ} 10^{\prime} 7^{\prime \prime} \mathrm{W}\end{array}$ & ISN1139 & $\begin{array}{l}\text { Nogueira, I.S., } \\
\text { Martins, L.L., da } \\
\text { Silva, W.J., Uto, F.N. }\end{array}$ & Periphyton \\
\hline UFG29973 & $\begin{array}{l}\text { Brazil, State of Goiás, } \\
\text { Britânia, Água Limpa River, } \\
\text { Água Limpa Farm, Site 1, } \\
\text { under macrophytes }\end{array}$ & $04 / 06 / 2004$ & $\begin{array}{l}15^{\circ} 18^{\prime} 58^{\prime \prime} \mathrm{S} ; \\
51^{\circ} 9^{\prime} 56^{\prime \prime} \mathrm{W}\end{array}$ & ISN873 & $\begin{array}{l}\text { Nogueira, I.S., } \\
\text { Martins, L.L., da } \\
\text { Silva, W.J., Nabout, } \\
\text { J.C. }\end{array}$ & Periphyton \\
\hline UFG43636 & $\begin{array}{l}\text { Brazil, State of Goiás, } \\
\text { Britânia, Água Limpa River, } \\
\text { Água Limpa Farm, Site } 1\end{array}$ & $17 / 07 / 2008$ & $\begin{array}{l}15^{\circ} 18^{\prime} 58^{\prime \prime} \mathrm{S} ; \\
51^{\circ} 9^{\prime} 56^{\prime \prime} \mathrm{W}\end{array}$ & ISN1226 & Nogueira, I.S. & Phytoplankton \\
\hline UFG43637 & $\begin{array}{l}\text { Brazil, State of Goiás, } \\
\text { Britânia, Lago dos Tigres, } \\
\text { Perdidas Island, Site } 2\end{array}$ & $17 / 07 / 2008$ & $\begin{array}{l}15^{\circ} 17^{\prime} 57^{\prime \prime} \mathrm{S} ; \\
51^{\circ} 10^{\prime} 7^{\prime \prime} \mathrm{W}\end{array}$ & ISN1227 & Nogueira, I.S. & Phytoplankton \\
\hline UFG43638 & $\begin{array}{l}\text { Brazil, State of Goiás, } \\
\text { Britânia, Lago dos Tigres, } \\
\text { outfall of Luanda Stream, } \\
\text { Santo Antônio Farm, Site } 3\end{array}$ & $17 / 07 / 2008$ & $\begin{array}{l}15^{\circ} 16^{\prime} 47^{\prime \prime} \mathrm{S} ; \\
51^{\circ} 9^{\prime} 44^{\prime \prime} \mathrm{W}\end{array}$ & ISN1228 & Nogueira, I.S. & Phytoplankton \\
\hline
\end{tabular}


Table 2. Continued.

\begin{tabular}{|c|c|c|c|c|c|c|}
\hline $\begin{array}{l}\text { Herbarium } \\
\text { number }\end{array}$ & Locality & Date & Coordinates & $\begin{array}{l}\text { Collector } \\
\text { number }\end{array}$ & Collectors & Habitat \\
\hline UFG43639 & $\begin{array}{l}\text { Brazil, State of Goiás, } \\
\text { Britânia, Lago dos Tigres, } \\
\text { Santo Antônio Farm, Site } 4\end{array}$ & $17 / 07 / 2008$ & $\begin{array}{l}15^{\circ} 16^{\prime} 17^{\prime \prime} \mathrm{S} \\
51^{\circ} 9^{\prime} 10^{\prime \prime} \mathrm{W}\end{array}$ & ISN1229 & Nogueira, I.S. & Phytoplankton \\
\hline UFG43640 & $\begin{array}{l}\text { Brazil, State of Goiás, } \\
\text { Britânia, Lago dos Tigres, } \\
\text { outfall of Arco-Íris Stream, } \\
\text { Site } 5\end{array}$ & $17 / 07 / 2008$ & $\begin{array}{l}15^{\circ} 15^{\prime} 46^{\prime \prime} \mathrm{S} \\
51^{\circ} 8^{\prime} 41^{\prime \prime} \mathrm{W}\end{array}$ & ISN1230 & Nogueira, I.S. & Phytoplankton \\
\hline UFG43641 & $\begin{array}{l}\text { Brazil, State of Goiás, } \\
\text { Britânia, Lago dos Tigres, } \\
\text { harbor ferry, Site } 6\end{array}$ & $17 / 07 / 2008$ & $\begin{array}{l}15^{\circ} 15^{\prime} 12^{\prime \prime} \mathrm{S} \\
51^{\circ} 8^{\prime} 33^{\prime \prime} \mathrm{W}\end{array}$ & ISN1231 & Nogueira, I.S. & Phytoplankton \\
\hline UFG43642 & $\begin{array}{l}\text { Brazil, State of Goiás, } \\
\text { Britânia, Lago dos Tigres, } \\
\text { Lago dos Tigres Farm, Site } 7\end{array}$ & $17 / 07 / 2008$ & $\begin{array}{l}15^{\circ} 14^{\prime} 17^{\prime \prime} \mathrm{S} \\
51^{\circ} 8^{\prime} 59^{\prime \prime} \mathrm{W}\end{array}$ & ISN1232 & Nogueira, I.S. & Phytoplankton \\
\hline UFG43643 & $\begin{array}{l}\text { Brazil, State of Goiás, } \\
\text { Britânia, Lago dos Tigres, city } \\
\text { in front of the Christ, Site } 8\end{array}$ & $17 / 07 / 2008$ & $\begin{array}{l}15^{\circ} 14^{\prime} 9^{\prime \prime} \mathrm{S} ; \\
51^{\circ} 9^{\prime} 27^{\prime \prime} \mathrm{W}\end{array}$ & ISN1233 & Nogueira, I.S. & Phytoplankton \\
\hline UFG43644 & $\begin{array}{l}\text { Brazil, State of Goiás, } \\
\text { Britânia, Lago dos Tigres, } \\
\text { downstream of the "lake", } \\
\text { Lago dos Tigres Farm, Site } 9\end{array}$ & $18 / 07 / 2008$ & $\begin{array}{l}15^{\circ} 13^{\prime} 43^{\prime \prime} \mathrm{S} \\
51^{\circ} 9^{\prime} 14^{\prime \prime} \mathrm{W}\end{array}$ & ISN1234 & Nogueira, I.S. & Phytoplankton \\
\hline UFG43645 & $\begin{array}{l}\text { Brazil, State of Goiás, } \\
\text { Britânia, Lago dos Tigres, } \\
\text { outfall of the "lake" in } \\
\text { Vermelho River, Site } 10\end{array}$ & $18 / 07 / 2008$ & $\begin{array}{l}15^{\circ} 13^{\prime} 18^{\prime \prime} \mathrm{S} \\
51^{\circ} 10^{\prime} 6^{\prime \prime} \mathrm{W}\end{array}$ & ISN1235 & Nogueira, I.S. & Phytoplankton \\
\hline UFG43646 & $\begin{array}{l}\text { Brazil, State of Goiás, } \\
\text { Britânia, Lago dos Tigres, } \\
\text { new channel of the Vermelho } \\
\text { River, Site } 11\end{array}$ & $18 / 07 / 2008$ & $\begin{array}{l}15^{\circ} 11^{\prime} 47^{\prime \prime} \mathrm{S} \\
51^{\circ} 9^{\prime} 57^{\prime \prime} \mathrm{W}\end{array}$ & ISN1236 & Nogueira, I.S. & Phytoplankton \\
\hline UFG43649 & $\begin{array}{l}\text { Brazil, State of Goiás, } \\
\text { Britânia, Água Limpa River, } \\
\text { Água Limpa Farm, Site } 1\end{array}$ & $19 / 08 / 2008$ & $\begin{array}{l}15^{\circ} 18^{\prime} 58^{\prime \prime} \mathrm{S} \\
51^{\circ} 9^{\prime} 56^{\prime \prime} \mathrm{W}\end{array}$ & ISN1239 & Nogueira, I.S. & Phytoplankton \\
\hline UFG43650 & $\begin{array}{l}\text { Brazil, State of Goiás, } \\
\text { Britânia, Lago dos Tigres, } \\
\text { Perdidas Island, Site } 2\end{array}$ & $19 / 08 / 2008$ & $\begin{array}{l}15^{\circ} 17^{\prime} 57^{\prime \prime} \mathrm{S} \\
51^{\circ} 10^{\prime} 7^{\prime \prime} \mathrm{W}\end{array}$ & ISN1240 & Nogueira, I.S. & Phytoplankton \\
\hline UFG43651 & $\begin{array}{l}\text { Brazil, State of Goiás, } \\
\text { Britânia, Lago dos Tigres, } \\
\text { outfall of Luanda Stream, } \\
\text { Santo Antônio Farm, Site } 3\end{array}$ & $19 / 08 / 2008$ & $\begin{array}{l}15^{\circ} 16^{\prime} 47^{\prime \prime} \mathrm{S} \\
51^{\circ} 9^{\prime} 44^{\prime \prime} \mathrm{W}\end{array}$ & ISN1241 & Nogueira, I.S. & Phytoplankton \\
\hline UFG43652 & $\begin{array}{l}\text { Brazil, State of Goiás, } \\
\text { Britânia, Lago dos Tigres, } \\
\text { Santo Antônio Farm, Site } 4\end{array}$ & $19 / 08 / 2008$ & $\begin{array}{l}15^{\circ} 16^{\prime} 17^{\prime \prime} \mathrm{S} \\
51^{\circ} 9^{\prime} 10^{\prime \prime} \mathrm{W}\end{array}$ & ISN1242 & Nogueira, I.S. & Phytoplankton \\
\hline UFG43653 & $\begin{array}{l}\text { Brazil, State of Goiás, } \\
\text { Britânia, Lago dos Tigres, } \\
\text { outfall of Arco-Íris Stream, } \\
\text { Site } 5\end{array}$ & $19 / 08 / 2008$ & $\begin{array}{l}15^{\circ} 15^{\prime} 46^{\prime \prime} \mathrm{S} \\
51^{\circ} 8^{\prime} 41^{\prime \prime} \mathrm{W}\end{array}$ & ISN1243 & Nogueira, I.S. & Phytoplankton \\
\hline UFG43654 & $\begin{array}{l}\text { Brazil, State of Goiás, } \\
\text { Britânia, Lago dos Tigres, } \\
\text { harbor ferry, Site } 6\end{array}$ & $19 / 08 / 2008$ & $\begin{array}{l}15^{\circ} 15^{\prime} 12^{\prime \prime} \mathrm{S} \\
51^{\circ} 8^{\prime} 33^{\prime \prime} \mathrm{W}\end{array}$ & ISN1244 & Nogueira, I.S. & Phytoplankton \\
\hline UFG43655 & $\begin{array}{l}\text { Brazil, State of Goiás, } \\
\text { Britânia, Lago dos Tigres, } \\
\text { Lago dos Tigres Farm, Site } 7\end{array}$ & $19 / 08 / 2008$ & $\begin{array}{l}15^{\circ} 14^{\prime} 17^{\prime \prime} \mathrm{S} ; \\
51^{\circ} 8^{\prime} 59^{\prime \prime} \mathrm{W}\end{array}$ & ISN1245 & Nogueira, I.S. & Phytoplankton \\
\hline UFG43656 & $\begin{array}{l}\text { Brazil, State of Goiás, } \\
\text { Britânia, Lago dos Tigres, city } \\
\text { in front of the Christ, Site } 8\end{array}$ & $19 / 08 / 2008$ & $\begin{array}{l}15^{\circ} 14^{\prime} 9^{\prime \prime} \mathrm{S} ; \\
51^{\circ} 9^{\prime} 27^{\prime \prime} \mathrm{W}\end{array}$ & ISN1246 & Nogueira, I.S. & Phytoplankton \\
\hline
\end{tabular}


Table 2. Continued.

\begin{tabular}{|c|c|c|c|c|c|c|}
\hline $\begin{array}{l}\text { Herbarium } \\
\text { number }\end{array}$ & Locality & Date & Coordinates & $\begin{array}{c}\text { Collector } \\
\text { number }\end{array}$ & Collectors & Habitat \\
\hline UFG43657 & $\begin{array}{l}\text { Brazil, State of Goiás, } \\
\text { Britânia, Lago dos Tigres, } \\
\text { downstream of the "lake", } \\
\text { Lago dos Tigres Farm, Site } 9\end{array}$ & $20 / 08 / 2008$ & $\begin{array}{l}15^{\circ} 13^{\prime} 43^{\prime \prime} \mathrm{S} ; \\
51^{\circ} 9^{\prime} 14^{\prime \prime} \mathrm{W}\end{array}$ & ISN1247 & Nogueira, I.S. & Phytoplankton \\
\hline UFG43658 & $\begin{array}{l}\text { Brazil, State of Goiás, } \\
\text { Britânia, Lago dos Tigres, } \\
\text { outfall of the "lake" in } \\
\text { Vermelho River, Site } 10\end{array}$ & $20 / 08 / 2008$ & $\begin{array}{l}15^{\circ} 13^{\prime} 18^{\prime \prime} \mathrm{S} ; \\
51^{\circ} 10^{\prime} 6^{\prime \prime} \mathrm{W}\end{array}$ & ISN1248 & Nogueira, I.S. & Phytoplankton \\
\hline UFG43659 & $\begin{array}{l}\text { Brazil, State of Goiás, } \\
\text { Britânia, Lago dos Tigres, } \\
\text { new channel of the Vermelho } \\
\text { River, Site } 11\end{array}$ & $20 / 08 / 2008$ & $\begin{array}{l}15^{\circ} 11^{\prime} 47^{\prime \prime} \mathrm{S} ; \\
51^{\circ} 9^{\prime} 57^{\prime \prime} \mathrm{W}\end{array}$ & ISN1249 & Nogueira, I.S. & Phytoplankton \\
\hline UFG43662 & $\begin{array}{l}\text { Brazil, State of Goiás, } \\
\text { Britânia, Água Limpa River, } \\
\text { Água Limpa Farm, Site } 1\end{array}$ & $16 / 09 / 2008$ & $\begin{array}{l}15^{\circ} 18^{\prime} 58^{\prime \prime} \mathrm{S} ; \\
51^{\circ} 9^{\prime} 56^{\prime \prime} \mathrm{W}\end{array}$ & ISN1252 & Nogueira, I.S. & Phytoplankton \\
\hline UFG43663 & $\begin{array}{l}\text { Brazil, State of Goiás, } \\
\text { Britânia, Lago dos Tigres, } \\
\text { Perdidas Island, Site } 2\end{array}$ & $16 / 09 / 2008$ & $\begin{array}{l}15^{\circ} 17^{\prime} 57^{\prime \prime} \mathrm{S} ; \\
51^{\circ} 10^{\prime} 7^{\prime \prime} \mathrm{W}\end{array}$ & ISN1253 & Nogueira, I.S. & Phytoplankton \\
\hline UFG43664 & $\begin{array}{l}\text { Brazil, State of Goiás, } \\
\text { Britânia, Lago dos Tigres, } \\
\text { outfall of Luanda Stream, } \\
\text { Santo Antônio Farm, Site } 3\end{array}$ & $16 / 09 / 2008$ & $\begin{array}{l}15^{\circ} 16^{\prime} 47^{\prime \prime} \mathrm{S} ; \\
51^{\circ} 9^{\prime} 44^{\prime \prime} \mathrm{W}\end{array}$ & ISN1254 & Nogueira, I.S. & Phytoplankton \\
\hline UFG43665 & $\begin{array}{l}\text { Brazil, State of Goiás, } \\
\text { Britânia, Lago dos Tigres, } \\
\text { Santo Antônio Farm, Site } 4\end{array}$ & $16 / 09 / 2008$ & $\begin{array}{l}15^{\circ} 16^{\prime} 17^{\prime \prime} \mathrm{S} ; \\
51^{\circ} 9^{\prime} 10^{\prime \prime} \mathrm{W}\end{array}$ & ISN1255 & Nogueira, I.S. & Phytoplankton \\
\hline UFG43666 & $\begin{array}{l}\text { Brazil, State of Goiás, } \\
\text { Britânia, Lago dos Tigres, } \\
\text { outfall of Arco-Íris Stream, } \\
\text { Site } 5\end{array}$ & $16 / 09 / 2008$ & $\begin{array}{l}15^{\circ} 15^{\prime} 46^{\prime \prime} \mathrm{S} ; \\
51^{\circ} 8^{\prime} 41^{\prime \prime} \mathrm{W}\end{array}$ & ISN1256 & Nogueira, I.S. & Phytoplankton \\
\hline UFG43667 & $\begin{array}{l}\text { Brazil, State of Goiás, } \\
\text { Britânia, Lago dos Tigres, } \\
\text { harbor ferry, Site } 6\end{array}$ & $16 / 09 / 2008$ & $\begin{array}{l}15^{\circ} 15^{\prime} 12^{\prime \prime} \mathrm{S} ; \\
51^{\circ} 8^{\prime} 33^{\prime \prime} \mathrm{W}\end{array}$ & ISN1257 & Nogueira, I.S. & Phytoplankton \\
\hline UFG43668 & $\begin{array}{l}\text { Brazil, State of Goiás, } \\
\text { Britânia, Lago dos Tigres, } \\
\text { Lago dos Tigres Farm, Site } 7\end{array}$ & $16 / 09 / 2008$ & $\begin{array}{l}15^{\circ} 14^{\prime} 17^{\prime \prime} \mathrm{S} ; \\
51^{\circ} 8^{\prime} 59^{\prime \prime} \mathrm{W}\end{array}$ & ISN1258 & Nogueira, I.S. & Phytoplankton \\
\hline UFG43669 & $\begin{array}{l}\text { Brazil, State of Goiás, } \\
\text { Britânia, Lago dos Tigres, city } \\
\text { in front of the Christ, Site } 8\end{array}$ & $16 / 09 / 2008$ & $\begin{array}{l}15^{\circ} 14^{\prime} 9^{\prime \prime} \mathrm{S} \\
51^{\circ} 9^{\prime} 27^{\prime \prime} \mathrm{W}\end{array}$ & ISN1259 & Nogueira, I.S. & Phytoplankton \\
\hline UFG43670 & $\begin{array}{l}\text { Brazil, State of Goiás, Britânia, } \\
\text { Lago dos Tigres, downstream of } \\
\text { the "lake", Lago dos Tigres } \\
\text { Farm, Site } 9\end{array}$ & $17 / 09 / 2008$ & $\begin{array}{l}15^{\circ} 13^{\prime} 43^{\prime \prime} \mathrm{S} ; \\
51^{\circ} 9^{\prime} 14^{\prime \prime} \mathrm{W}\end{array}$ & ISN1260 & Nogueira, I.S. & Phytoplankton \\
\hline UFG43671 & $\begin{array}{l}\text { Brazil, State of Goiás, } \\
\text { Britânia, Lago dos Tigres, } \\
\text { outfall of the "lake" in } \\
\text { Vermelho River, Site } 10\end{array}$ & $17 / 09 / 2008$ & $\begin{array}{l}15^{\circ} 13^{\prime} 18^{\prime \prime} \mathrm{S} \\
51^{\circ} 10^{\prime} 6^{\prime \prime} \mathrm{W}\end{array}$ & ISN1261 & Nogueira, I.S. & Phytoplankton \\
\hline UFG43672 & $\begin{array}{l}\text { Brazil, State of Goiás, } \\
\text { Britânia, Lago dos Tigres, } \\
\text { new channel of the Vermelho } \\
\text { River, Site } 11\end{array}$ & $17 / 09 / 2008$ & $\begin{array}{l}15^{\circ} 11^{\prime} 47^{\prime \prime} \mathrm{S} ; \\
51^{\circ} 9^{\prime} 57^{\prime \prime} \mathrm{W}\end{array}$ & ISN1262 & Nogueira, I.S. & Phytoplankton \\
\hline UFG43675 & $\begin{array}{l}\text { Brazil, State of Goiás, } \\
\text { Britânia, Água Limpa River, } \\
\text { Água Limpa Farm, Site } 1\end{array}$ & 19/10/2008 & $\begin{array}{l}15^{\circ} 18^{\prime} 58^{\prime \prime} \mathrm{S} ; \\
51^{\circ} 9^{\prime} 56^{\prime \prime} \mathrm{W}\end{array}$ & ISN1265 & Nogueira, I.S. & Phytoplankton \\
\hline UFG43676 & $\begin{array}{l}\text { Brazil, State of Goiás, } \\
\text { Britânia, Lago dos Tigres, } \\
\text { Perdidas Island, Site } 2\end{array}$ & $19 / 10 / 2008$ & $\begin{array}{l}15^{\circ} 17^{\prime} 57^{\prime \prime} \mathrm{S} ; \\
51^{\circ} 10^{\prime} 7^{\prime \prime} \mathrm{W}\end{array}$ & ISN1266 & Nogueira, I.S. & Phytoplankton \\
\hline
\end{tabular}


Table 2. Continued.

\begin{tabular}{|c|c|c|c|c|c|c|}
\hline $\begin{array}{l}\text { Herbarium } \\
\text { number }\end{array}$ & Locality & Date & Coordinates & $\begin{array}{l}\text { Collector } \\
\text { number }\end{array}$ & Collectors & Habitat \\
\hline UFG43677 & $\begin{array}{l}\text { Brazil, State of Goiás, } \\
\text { Britânia, Lago dos Tigres, } \\
\text { outfall of Luanda Stream, } \\
\text { Santo Antônio Farm, Site } 3\end{array}$ & $19 / 10 / 2008$ & $\begin{array}{l}15^{\circ} 16^{\prime} 47^{\prime \prime} \mathrm{S} \\
51^{\circ} 9^{\prime} 44^{\prime \prime} \mathrm{W}\end{array}$ & ISN1267 & Nogueira, I.S. & Phytoplankton \\
\hline UFG43678 & $\begin{array}{l}\text { Brazil, State of Goiás, } \\
\text { Britânia, Lago dos Tigres, } \\
\text { Santo Antônio Farm, Site } 4\end{array}$ & $19 / 10 / 2008$ & $\begin{array}{l}15^{\circ} 16^{\prime} 17^{\prime \prime} \mathrm{S} ; \\
51^{\circ} 9^{\prime} 10^{\prime \prime} \mathrm{W}\end{array}$ & ISN1268 & Nogueira, I.S. & Phytoplankton \\
\hline UFG43679 & $\begin{array}{l}\text { Brazil, State of Goiás, } \\
\text { Britânia, Lago dos Tigres, } \\
\text { outfall of Arco-Íris Stream, } \\
\text { Site } 5\end{array}$ & $19 / 10 / 2008$ & $\begin{array}{l}15^{\circ} 15^{\prime} 46^{\prime \prime} \mathrm{S} ; \\
51^{\circ} 8^{\prime} 41^{\prime \prime} \mathrm{W}\end{array}$ & ISN1269 & Nogueira, I.S. & Phytoplankton \\
\hline UFG43680 & $\begin{array}{l}\text { Brazil, State of Goiás, } \\
\text { Britânia, Lago dos Tigres, } \\
\text { harbor ferry, Site } 6\end{array}$ & $19 / 10 / 2008$ & $\begin{array}{l}15^{\circ} 15^{\prime} 12^{\prime \prime} \mathrm{S} ; \\
51^{\circ} 8^{\prime} 33^{\prime \prime} \mathrm{W}\end{array}$ & ISN1270 & Nogueira, I.S. & Phytoplankton \\
\hline UFG43681 & $\begin{array}{l}\text { Brazil, State of Goiás, } \\
\text { Britânia, Lago dos Tigres, } \\
\text { Lago dos Tigres Farm, Site } 7\end{array}$ & $19 / 10 / 2008$ & $\begin{array}{l}15^{\circ} 14^{\prime} 17^{\prime \prime} \mathrm{S} ; \\
51^{\circ} 8^{\prime} 59^{\prime \prime} \mathrm{W}\end{array}$ & ISN1271 & Nogueira, I.S. & Phytoplankton \\
\hline UFG43682 & $\begin{array}{l}\text { Brazil, State of Goiás, } \\
\text { Britânia, Lago dos Tigres, city } \\
\text { in front of the Christ, Site } 8\end{array}$ & $19 / 10 / 2008$ & $\begin{array}{l}15^{\circ} 14^{\prime} 9^{\prime \prime} \mathrm{S} ; \\
51^{\circ} 9^{\prime} 27^{\prime \prime} \mathrm{W}\end{array}$ & ISN1272 & Nogueira, I.S. & Phytoplankton \\
\hline UFG43683 & $\begin{array}{l}\text { Brazil, State of Goiás, Britânia, } \\
\text { Lago dos Tigres, downstream of } \\
\text { the "lake", Lago dos Tigres } \\
\text { Farm, Site } 9\end{array}$ & $20 / 10 / 2008$ & $\begin{array}{l}15^{\circ} 13^{\prime} 43^{\prime \prime} \mathrm{S} ; \\
51^{\circ} 9^{\prime} 14^{\prime \prime} \mathrm{W}\end{array}$ & ISN1273 & Nogueira, I.S. & Phytoplankton \\
\hline UFG43684 & $\begin{array}{l}\text { Brazil, State of Goiás, } \\
\text { Britânia, Lago dos Tigres, } \\
\text { outfall of the "lake" in } \\
\text { Vermelho River, Site } 10\end{array}$ & $20 / 10 / 2008$ & $\begin{array}{l}15^{\circ} 13^{\prime} 18^{\prime \prime} \mathrm{S} ; \\
51^{\circ} 10^{\prime} 6^{\prime \prime} \mathrm{W}\end{array}$ & ISN1274 & Nogueira, I.S. & Phytoplankton \\
\hline UFG43685 & $\begin{array}{l}\text { Brazil, State of Goiás, } \\
\text { Britânia, Lago dos Tigres, } \\
\text { new channel of the Vermelho } \\
\text { River, Site } 11\end{array}$ & $20 / 10 / 2008$ & $\begin{array}{l}15^{\circ} 11^{\prime} 47^{\prime \prime} \mathrm{S} ; \\
51^{\circ} 9^{\prime} 57^{\prime \prime} \mathrm{W}\end{array}$ & ISN1275 & Nogueira, I.S. & Phytoplankton \\
\hline UFG43688 & $\begin{array}{l}\text { Brazil, State of Goiás, } \\
\text { Britânia, Água Limpa River, } \\
\text { Água Limpa Farm, Site } 1\end{array}$ & $15 / 11 / 2008$ & $\begin{array}{l}15^{\circ} 18^{\prime} 58^{\prime \prime} \mathrm{S} ; \\
51^{\circ} 9^{\prime} 56^{\prime \prime} \mathrm{W}\end{array}$ & ISN1278 & Nogueira, I.S. & Phytoplankton \\
\hline UFG43689 & $\begin{array}{l}\text { Brazil, State of Goiás, } \\
\text { Britânia, Lago dos Tigres, } \\
\text { Perdidas Island, Site } 2\end{array}$ & $15 / 11 / 2008$ & $\begin{array}{l}15^{\circ} 17^{\prime} 57^{\prime \prime} \mathrm{S} ; \\
51^{\circ} 10^{\prime} 7^{\prime \prime} \mathrm{W}\end{array}$ & ISN1279 & Nogueira, I.S. & Phytoplankton \\
\hline UFG43690 & $\begin{array}{l}\text { Brazil, State of Goiás, } \\
\text { Britânia, Lago dos Tigres, } \\
\text { outfall of Luanda Stream, } \\
\text { Santo Antônio Farm, Site } 3\end{array}$ & $15 / 11 / 2008$ & $\begin{array}{l}15^{\circ} 16^{\prime} 47^{\prime \prime} \mathrm{S} ; \\
51^{\circ} 9^{\prime} 44^{\prime \prime} \mathrm{W}\end{array}$ & ISN1280 & Nogueira, I.S. & Phytoplankton \\
\hline UFG43691 & $\begin{array}{l}\text { Brazil, State of Goiás, } \\
\text { Britânia, Lago dos Tigres, } \\
\text { Santo Antônio Farm, Site } 4\end{array}$ & $15 / 11 / 2008$ & $\begin{array}{l}15^{\circ} 16^{\prime} 17^{\prime \prime} \mathrm{S} ; \\
51^{\circ} 9^{\prime} 10^{\prime \prime} \mathrm{W}\end{array}$ & ISN1281 & Nogueira, I.S. & Phytoplankton \\
\hline UFG43692 & $\begin{array}{l}\text { Brazil, State of Goiás, } \\
\text { Britânia, Lago dos Tigres, } \\
\text { outfall of Arco-Íris Stream, } \\
\text { Site } 5\end{array}$ & $15 / 11 / 2008$ & $\begin{array}{l}15^{\circ} 15^{\prime} 46^{\prime \prime} \mathrm{S} \\
51^{\circ} 8^{\prime} 41^{\prime \prime} \mathrm{W}\end{array}$ & ISN1282 & Nogueira, I.S. & Phytoplankton \\
\hline UFG43693 & $\begin{array}{l}\text { Brazil, State of Goiás, } \\
\text { Britânia, Lago dos Tigres, } \\
\text { harbor ferry, Site } 6\end{array}$ & $15 / 11 / 2008$ & $\begin{array}{l}15^{\circ} 15^{\prime} 12^{\prime \prime} \mathrm{S} ; \\
51^{\circ} 8^{\prime} 33^{\prime \prime} \mathrm{W}\end{array}$ & ISN1283 & Nogueira, I.S. & Phytoplankton \\
\hline UFG43694 & $\begin{array}{l}\text { Brazil, State of Goiás, } \\
\text { Britânia, Lago dos Tigres, } \\
\text { Lago dos Tigres Farm, Site } 7\end{array}$ & $15 / 11 / 2008$ & $\begin{array}{l}15^{\circ} 14^{\prime} 17^{\prime \prime} \mathrm{S} ; \\
51^{\circ} 8^{\prime} 59^{\prime \prime} \mathrm{W}\end{array}$ & ISN1284 & Nogueira, I.S. & Phytoplankton \\
\hline
\end{tabular}


Table 2. Continued.

\begin{tabular}{|c|c|c|c|c|c|c|}
\hline $\begin{array}{l}\text { Herbarium } \\
\text { number }\end{array}$ & Locality & Date & Coordinates & $\begin{array}{l}\text { Collector } \\
\text { number }\end{array}$ & Collectors & Habitat \\
\hline UFG43695 & $\begin{array}{l}\text { Brazil, State of Goiás, } \\
\text { Britânia, Lago dos Tigres, city } \\
\text { in front of the Christ, Site } 8\end{array}$ & $15 / 11 / 2008$ & $\begin{array}{l}15^{\circ} 14^{\prime} 9^{\prime \prime} \mathrm{S} ; \\
51^{\circ} 9^{\prime} 27^{\prime \prime} \mathrm{W}\end{array}$ & ISN1285 & Nogueira, I.S. & Phytoplankton \\
\hline UFG43696 & $\begin{array}{l}\text { Brazil, State of Goiás, } \\
\text { Britânia, Lago dos Tigres, } \\
\text { downstream of the "lake", } \\
\text { Lago dos Tigres Farm, Site } 9\end{array}$ & $16 / 11 / 2008$ & $\begin{array}{l}15^{\circ} 13^{\prime} 43^{\prime \prime} \mathrm{S} ; \\
51^{\circ} 9^{\prime} 14^{\prime \prime} \mathrm{W}\end{array}$ & ISN1286 & Nogueira, I.S. & Phytoplankton \\
\hline UFG43697 & $\begin{array}{l}\text { Brazil, State of Goiás, } \\
\text { Britânia, Lago dos Tigres, } \\
\text { outfall of the "lake" in } \\
\text { Vermelho River, Site } 10\end{array}$ & $16 / 11 / 2008$ & $\begin{array}{l}15^{\circ} 13^{\prime} 18^{\prime \prime} \mathrm{S} \\
51^{\circ} 10^{\prime} 6^{\prime \prime} \mathrm{W}\end{array}$ & ISN1287 & Nogueira, I.S. & Phytoplankton \\
\hline UFG43698 & $\begin{array}{l}\text { Brazil, State of Goiás, } \\
\text { Britânia, Lago dos Tigres, } \\
\text { new channel of the Vermelho } \\
\text { River, Site } 11\end{array}$ & $16 / 11 / 2008$ & $\begin{array}{l}15^{\circ} 11^{\prime} 47^{\prime \prime} \mathrm{S} ; \\
51^{\circ} 9^{\prime} 57^{\prime \prime} \mathrm{W}\end{array}$ & ISN1288 & Nogueira, I.S. & Phytoplankton \\
\hline UFG43701 & $\begin{array}{l}\text { Brazil, State of Goiás, } \\
\text { Britânia, Água Limpa River, } \\
\text { Água Limpa Farm, Site } 1\end{array}$ & $11 / 12 / 2008$ & $\begin{array}{l}15^{\circ} 18^{\prime} 58^{\prime \prime} \mathrm{S} \\
51^{\circ} 9^{\prime} 56^{\prime \prime} \mathrm{W}\end{array}$ & ISN1291 & Nogueira, I.S. & Phytoplankton \\
\hline UFG43702 & $\begin{array}{l}\text { Brazil, State of Goiás, } \\
\text { Britânia, Lago dos Tigres, } \\
\text { Perdidas Island, Site } 2\end{array}$ & $11 / 12 / 2008$ & $\begin{array}{l}15^{\circ} 17^{\prime} 57^{\prime \prime} \mathrm{S} ; \\
51^{\circ} 10^{\prime} 7^{\prime \prime} \mathrm{W}\end{array}$ & ISN1292 & Nogueira, I.S. & Phytoplankton \\
\hline UFG43703 & $\begin{array}{l}\text { Brazil, State of Goiás, } \\
\text { Britânia, Lago dos Tigres, } \\
\text { outfall of Luanda Stream, } \\
\text { Santo Antônio Farm, Site } 3\end{array}$ & $11 / 12 / 2008$ & $\begin{array}{l}15^{\circ} 16^{\prime} 47^{\prime \prime} \mathrm{S} \\
51^{\circ} 9^{\prime} 44^{\prime \prime} \mathrm{W}\end{array}$ & ISN1293 & Nogueira, I.S. & Phytoplankton \\
\hline UFG43704 & $\begin{array}{l}\text { Brazil, State of Goiás, } \\
\text { Britânia, Lago dos Tigres, } \\
\text { Santo Antônio Farm, Site } 4\end{array}$ & $11 / 12 / 2008$ & $\begin{array}{l}15^{\circ} 16^{\prime} 17^{\prime \prime} \mathrm{S} ; \\
51^{\circ} 9^{\prime} 10^{\prime \prime} \mathrm{W}\end{array}$ & ISN1294 & Nogueira, I.S. & Phytoplankton \\
\hline UFG43705 & $\begin{array}{l}\text { Brazil, State of Goiás, } \\
\text { Britânia, Lago dos Tigres, } \\
\text { outfall of Arco-Íris Stream, } \\
\text { Site } 5\end{array}$ & $11 / 12 / 2008$ & $\begin{array}{l}15^{\circ} 15^{\prime} 46^{\prime \prime} \mathrm{S} \\
51^{\circ} 8^{\prime} 41^{\prime \prime} \mathrm{W}\end{array}$ & ISN1295 & Nogueira, I.S. & Phytoplankton \\
\hline UFG43706 & $\begin{array}{l}\text { Brazil, State of Goiás, } \\
\text { Britânia, Lago dos Tigres, } \\
\text { harbor ferry, Site } 6\end{array}$ & $11 / 12 / 2008$ & $\begin{array}{l}15^{\circ} 15^{\prime} 12^{\prime \prime} \mathrm{S} \\
51^{\circ} 8^{\prime} 33^{\prime \prime} \mathrm{W}\end{array}$ & ISN1296 & Nogueira, I.S. & Phytoplankton \\
\hline UFG43707 & $\begin{array}{l}\text { Brazil, State of Goiás, } \\
\text { Britânia, Lago dos Tigres, } \\
\text { Lago dos Tigres Farm, Site } 7\end{array}$ & $11 / 12 / 2008$ & $\begin{array}{l}15^{\circ} 14^{\prime} 17^{\prime \prime} \mathrm{S} \\
51^{\circ} 8^{\prime} 59^{\prime \prime} \mathrm{W}\end{array}$ & ISN1297 & Nogueira, I.S. & Phytoplankton \\
\hline UFG43708 & $\begin{array}{l}\text { Brazil, State of Goiás, } \\
\text { Britânia, Lago dos Tigres, city } \\
\text { in front of the Christ, Site } 8\end{array}$ & $11 / 12 / 2008$ & $\begin{array}{l}15^{\circ} 14^{\prime} 9^{\prime \prime} \mathrm{S} ; \\
51^{\circ} 9^{\prime} 27^{\prime \prime} \mathrm{W}\end{array}$ & ISN1298 & Nogueira, I.S. & Phytoplankton \\
\hline UFG43709 & $\begin{array}{l}\text { Brazil, State of Goiás, } \\
\text { Britânia, Lago dos Tigres, } \\
\text { outfall of the "lake" in } \\
\text { Vermelho River, Site } 9\end{array}$ & $12 / 12 / 2008$ & $\begin{array}{l}15^{\circ} 13^{\prime} 43^{\prime \prime} \mathrm{S} ; \\
51^{\circ} 9^{\prime} 14^{\prime \prime} \mathrm{W}\end{array}$ & ISN1299 & Nogueira, I.S. & Phytoplankton \\
\hline UFG43710 & $\begin{array}{l}\text { Brazil, State of Goiás, } \\
\text { Britânia, Lago dos Tigres, } \\
\text { outfall of the "lake" in } \\
\text { Vermelho River, Site } 10\end{array}$ & $12 / 12 / 2008$ & $\begin{array}{l}15^{\circ} 13^{\prime} 18^{\prime \prime} \mathrm{S} \\
51^{\circ} 10^{\prime} 6^{\prime \prime} \mathrm{W}\end{array}$ & ISN1300 & Nogueira, I.S. & Phytoplankton \\
\hline UFG43711 & $\begin{array}{l}\text { Brazil, State of Goiás, } \\
\text { Britânia, Lago dos Tigres, } \\
\text { new channel of the Vermelho } \\
\text { River, Site } 11\end{array}$ & $12 / 12 / 2008$ & $\begin{array}{l}15^{\circ} 11^{\prime} 47^{\prime \prime} \mathrm{S} ; \\
51^{\circ} 9^{\prime} 57^{\prime \prime} \mathrm{W}\end{array}$ & ISN1301 & Nogueira, I.S. & Phytoplankton \\
\hline UFG43714 & $\begin{array}{l}\text { Brazil, State of Goiás, } \\
\text { Britânia, Água Limpa River, } \\
\text { Água Limpa Farm, Site } 1\end{array}$ & $21 / 01 / 2009$ & $\begin{array}{l}15^{\circ} 18^{\prime} 58^{\prime \prime} \mathrm{S} \\
51^{\circ} 9^{\prime} 56^{\prime \prime} \mathrm{W}\end{array}$ & ISN1304 & Nogueira, I.S. & Phytoplankton \\
\hline
\end{tabular}


Table 2. Continued.

\begin{tabular}{|c|c|c|c|c|c|c|}
\hline $\begin{array}{l}\text { Herbarium } \\
\text { number }\end{array}$ & Locality & Date & Coordinates & $\begin{array}{l}\text { Collector } \\
\text { number }\end{array}$ & Collectors & Habitat \\
\hline UFG43715 & $\begin{array}{l}\text { Brazil, State of Goiás, } \\
\text { Britânia, Lago dos Tigres, } \\
\text { Perdidas Island, Site } 2\end{array}$ & 21/01/2009 & $\begin{array}{l}15^{\circ} 17^{\prime} 57^{\prime \prime} \mathrm{S} ; \\
51^{\circ} 10^{\prime} 7^{\prime \prime} \mathrm{W}\end{array}$ & ISN1305 & Nogueira, I.S. & Phytoplankton \\
\hline UFG43716 & $\begin{array}{l}\text { Brazil, State of Goiás, } \\
\text { Britânia, Lago dos Tigres, } \\
\text { outfall of Luanda Stream, } \\
\text { Santo Antônio Farm, Site } 3\end{array}$ & 21/01/2009 & $\begin{array}{l}15^{\circ} 16^{\prime} 47^{\prime \prime} \mathrm{S} \\
51^{\circ} 9^{\prime} 44^{\prime \prime} \mathrm{W}\end{array}$ & ISN1306 & Nogueira, I.S. & Phytoplankton \\
\hline UFG43717 & $\begin{array}{l}\text { Brazil, State of Goiás, } \\
\text { Britânia, Lago dos Tigres, } \\
\text { Santo Antônio Farm, Site } 4\end{array}$ & 21/01/2009 & $\begin{array}{l}15^{\circ} 16^{\prime} 17^{\prime \prime} \mathrm{S} ; \\
51^{\circ} 9^{\prime} 10^{\prime \prime} \mathrm{W}\end{array}$ & ISN1307 & Nogueira, I.S. & Phytoplankton \\
\hline UFG43718 & $\begin{array}{l}\text { Brazil, State of Goiás, } \\
\text { Britânia, Lago dos Tigres, } \\
\text { outfall of Arco-Íris Stream, } \\
\text { Site } 5\end{array}$ & 21/01/2009 & $\begin{array}{l}15^{\circ} 15^{\prime} 46^{\prime \prime} \mathrm{S} \\
51^{\circ} 8^{\prime} 41^{\prime \prime} \mathrm{W}\end{array}$ & ISN1308 & Nogueira, I.S. & Phytoplankton \\
\hline UFG43719 & $\begin{array}{l}\text { Brazil, State of Goiás, } \\
\text { Britânia, Lago dos Tigres, } \\
\text { harbor ferry, Site } 6\end{array}$ & 21/01/2009 & $\begin{array}{l}15^{\circ} 15^{\prime} 12^{\prime \prime} \mathrm{S} ; \\
51^{\circ} 8^{\prime} 33^{\prime \prime} \mathrm{W}\end{array}$ & ISN1309 & Nogueira, I.S. & Phytoplankton \\
\hline UFG43720 & $\begin{array}{l}\text { Brazil, State of Goiás, } \\
\text { Britânia, Lago dos Tigres, } \\
\text { Lago dos Tigres Farm, Site } 7\end{array}$ & 21/01/2009 & $\begin{array}{l}15^{\circ} 14^{\prime} 17^{\prime \prime} \mathrm{S} ; \\
51^{\circ} 8^{\prime} 59^{\prime \prime} \mathrm{W}\end{array}$ & ISN1310 & Nogueira, I.S. & Phytoplankton \\
\hline UFG43721 & $\begin{array}{l}\text { Brazil, State of Goiás, } \\
\text { Britânia, Lago dos Tigres, city } \\
\text { in front of the Christ, Site } 8\end{array}$ & 21/01/2009 & $\begin{array}{l}15^{\circ} 14^{\prime} 9^{\prime \prime} \mathrm{S} \\
51^{\circ} 9^{\prime} 27^{\prime \prime} \mathrm{W}\end{array}$ & ISN1311 & Nogueira, I.S. & Phytoplankton \\
\hline UFG43722 & $\begin{array}{l}\text { Brazil, State of Goiás, } \\
\text { Britânia, Lago dos Tigres, } \\
\text { downstream of the "lake", } \\
\text { Lago dos Tigres Farm, Site } 9\end{array}$ & $22 / 01 / 2009$ & $\begin{array}{l}15^{\circ} 13^{\prime} 43^{\prime \prime} \mathrm{S} ; \\
51^{\circ} 9^{\prime} 14^{\prime \prime} \mathrm{W}\end{array}$ & ISN1312 & Nogueira, I.S. & Phytoplankton \\
\hline UFG43723 & $\begin{array}{l}\text { Brazil, State of Goiás, } \\
\text { Britânia, Lago dos Tigres, } \\
\text { outfall of the "lake" in } \\
\text { Vermelho River, Site } 10\end{array}$ & $22 / 01 / 2009$ & $\begin{array}{l}15^{\circ} 13^{\prime} 18^{\prime \prime} \mathrm{S} ; \\
51^{\circ} 10^{\prime} 6^{\prime \prime} \mathrm{W}\end{array}$ & ISN1313 & Nogueira, I.S. & Phytoplankton \\
\hline UFG43724 & $\begin{array}{l}\text { Brazil, State of Goiás, } \\
\text { Britânia, Lago dos Tigres, } \\
\text { new channel of the Vermelho } \\
\text { River, Site } 11\end{array}$ & $22 / 01 / 2009$ & $\begin{array}{l}15^{\circ} 11^{\prime} 47^{\prime \prime} \mathrm{S} ; \\
51^{\circ} 9^{\prime} 57^{\prime \prime} \mathrm{W}\end{array}$ & ISN1314 & Nogueira, I.S. & Phytoplankton \\
\hline
\end{tabular}

Distribution. Brazil, State of São Paulo, São Paulo, Parque Estadual das Fontes do Ipiranga (Rocha \& Bicudo 2008); Pioneer citation for the State of Goiás.

Remarks. Krammer (2000, p. 55, 214) cited in the original description the specimens recorded on "Figs. 21: 8, 9" as representatives of $P$. acrophaeria var. tumidula. However, in the legend of these figures, Krammer (2000, p. 300) cited "Figs. 8, 9. Pinnularia acrosphaeria var. turgidula Grunow ex Cleve (p. 55)". Krammer (2000, p. 300) still described the specimens recorded on the figures 8 and 9 originally from "Nelubium pond in the botanical garden Munich, slide 1792 MR) which corresponds to the type and type locality of $P$. acrosphaeria var. tumidula (and not $P$. acrosphaeria var. turgidula), i.e., "Nelubium pond in the botanical garden Munich, slide 1792 MR" (see Krammer 2000, p. 55, 214). Because the agreement with the type and type locality and with the original description of the taxa, we concluded that the description of the legend is, actually, a typographic error that can be corrected. In contrast, if it was not accepted as typographic error, the taxon would not be valid because was published without an illustration as required by Art. 44.2 of the ICN (McNeill et al., 2012). Similarly, other authors seem to consider the legend of the Krammer (2000, figures 21: 8,9) as a typographical error since has adopted the name as validly proposed (e.g., Rocha \& Bicudo 2008, Montoya-Moreno et al. 2013).

The diacritical characteristic of this taxon from the nominated variety is the prominent intumescence at the middle part of the valve (Krammer 2000). The specimens found in Lago dos Tigres agreed with the original description and is very similar to the type material illustrated by Krammer (2000, figure 21: 9). Rocha \& Bicudo (2008) also found P. acrosphaeria var. tumidula in the State of São Paulo, which the specimens of the Lagos dos Tigres were similar.

All the taxa subscribed to $P$. acrophaeria present axial area with granulations (Krammer 2000). Although this feature was observed in the representatives of $P$. acrophaeria var. tumidula from Lago dos Tigres, it seems to be inconspicuous during the process of microphotography (Figure 2). This seems to be the case of the representatives recorded by Rocha \& Bicudo (2008, Figures 43, 44), in which the granulations were little evident in the pictures but this data was provided in the characterization of the species. 
da Silva, W.J. et al.

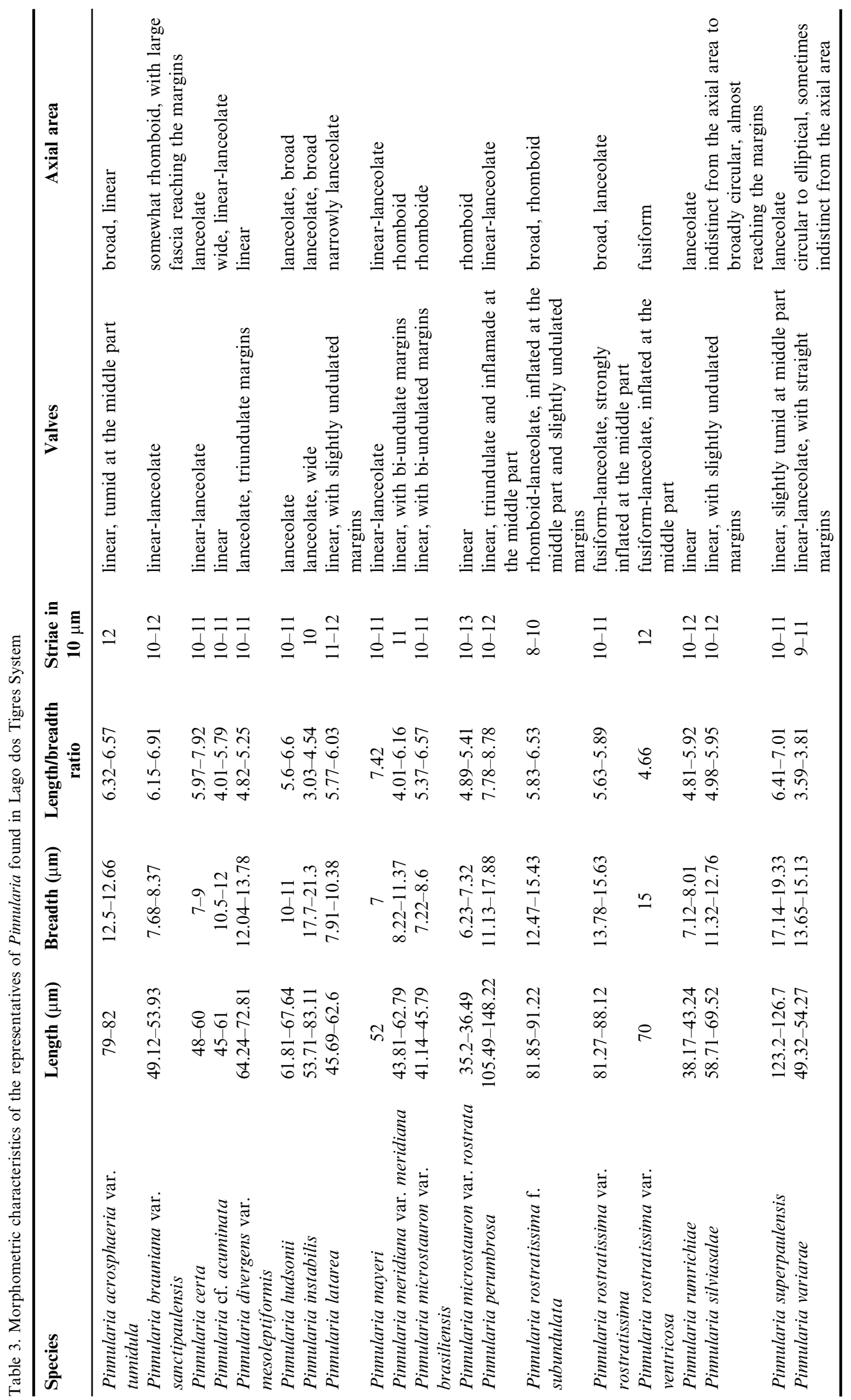


Pinnularia from Lago dos Tigres

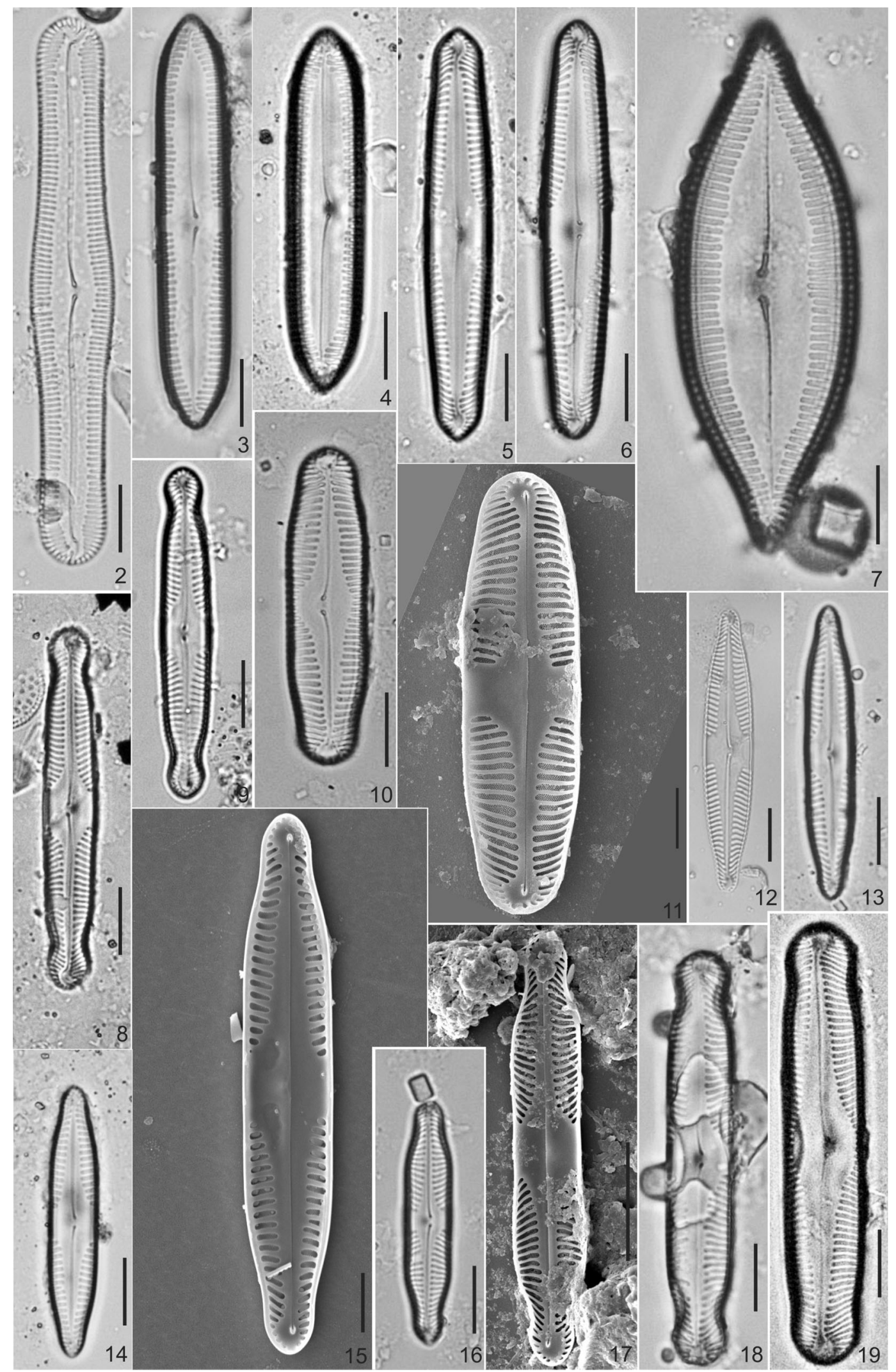

Figures 2-19. Species of Pinnularia from Lago dos Tigres (Figures 2-10, 12-14, 16, 18, 19: light microscopy; Figures 11, 15, 17: scanning electron microscopy); Figure 2. Pinnularia acrosphaeria var. tumidula; Figures 3, 4. Pinnularia acuminata; Figures 5, 6. Pinnularia hudsonii; Figure 7. Pinnularia instabilis; Figures 8, 9. Pinnularia mayeri, Figures 10, 11. Pinnularia meridiana var. meridiana; Figure 12. Pinnularia certa; Figure 13-15. Pinnularia microstauron var. brasiliensis; Figures 16-17. Pinnularia microstauron var. rostrata; Figure 18. Pinnularia latarea; Figure 19. Pinnularia silviasalae. Scale bar: Figures 2-10, 12-14, 16-19: $10 \mu \mathrm{m}$; Figures 11, 15: $5 \mu \mathrm{m}$. 


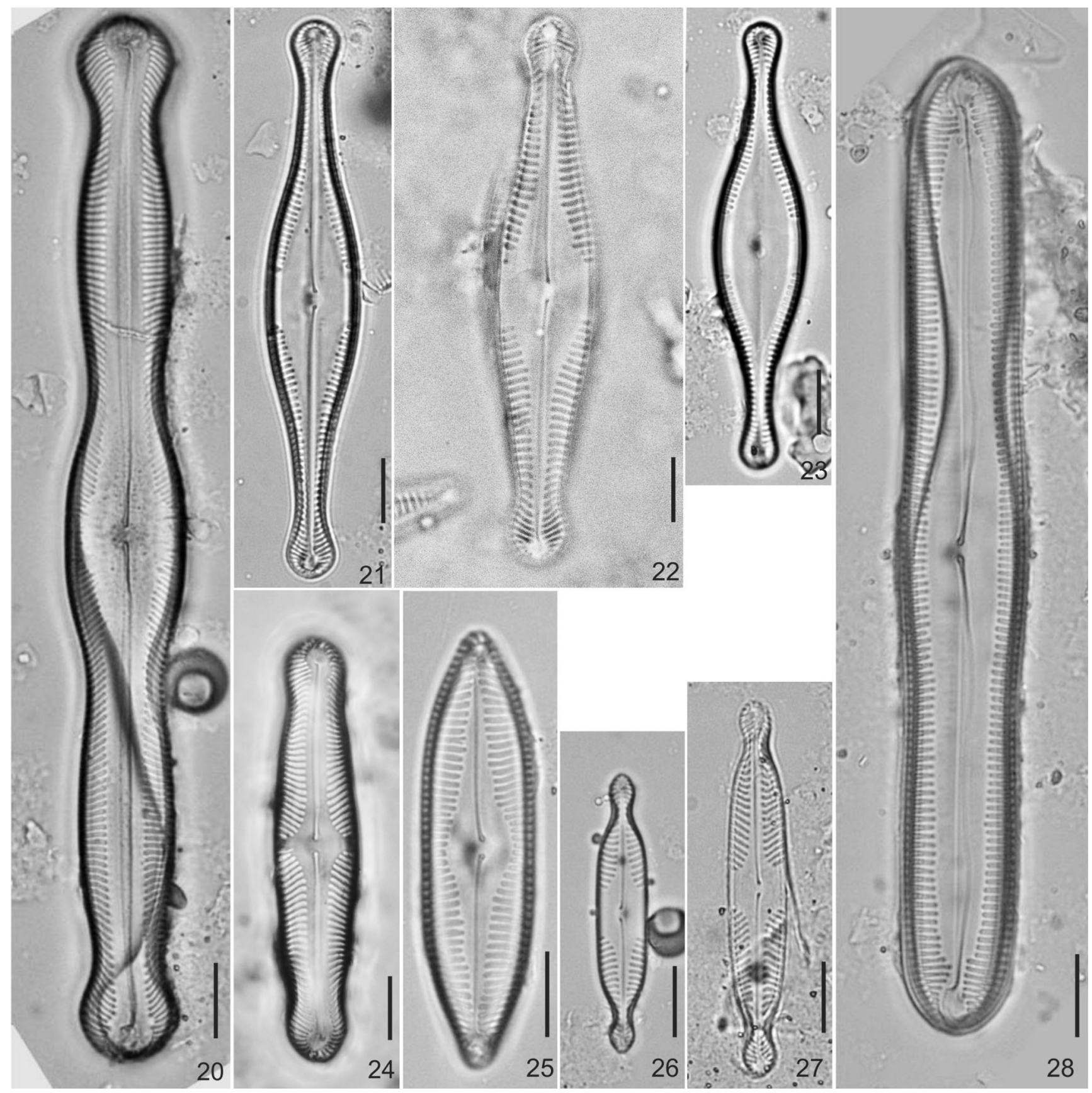

Figures 20-28. Species of Pinnularia from Lago dos Tigres (light microscopy); Figure 20. Pinnularia perumbrosa; Figure 21. Pinnularia rostratissima var. rostratissima; Figure 22. Pinnularia rostratissima f. subundulata; Figure 23. Pinnularia rostratissima var. ventricosa; Figure 24. Pinnularia divergens var. mesoleptiformis; Figure 25. Pinnularia variarae; Figure 26. Pinnularia rumrichiae; Figure 27. Pinnularia brauniana var. sanctipaulensis; Figure 28. Pinnularia superpaulensis. Scale bar: $10 \mu \mathrm{m}$.

\section{Pinnularia brauniana var. sanctipaulensis}

A.C.R. Rocha, Hoehnea, vol. 35, n. 4, p. 603, Figs. 21-25, 2008. (Figure 27)

Holotype. Preparation SP255743 in Herbário "Maria Eneyda P. Kauffmann Fidalgo" (SP) do Instituto de Botânica da Secretaria do Meio Ambiente do Estado de São Paulo.

Type locality. Lago das Ninféias, Parque Estadual das Fontes do Ipiranga, São Paulo, State of São Paulo, Brazil.

Valves linear-lanceolate; capitate apices; length: 49.12 $53.93 \mu \mathrm{m}$, breadth: $7.68-8.37 \mu \mathrm{m}$, length/breadth ratio: $6.15-$
6.91; axial area linear to linear-lanceolate; axial area somewhat rhomboid, with large fascia reaching the margins; raphe lateral, terminal fissures hooked, proximal fissures curved to the same side; striae radiated, becoming convergent towards to the ends of the valves; striae: $10-12 / 10 \mu \mathrm{m}$.

\section{Material examined}

Brazil, Goiás, Britânia, Lago dos Tigres, plankton, Nogueira et al., Site 11, 20/10/2008, P11M10, ISN1275 (UFG 43685). 


\section{Distribution}

Brazil, State of São Paulo, São Paulo, Parque Estadual das Fontes do Ipiranga (Rocha \& Bicudo 2008); Pioneer citation for the State.

\section{Pinnularia certa}

Krammer \& Metzeltin, Iconogr. Diatomol., vol. 5, p. 166, Figs. 178: 12-17, 1998. (Figure 12)

Holotype. Preparation 2607c KASSEL, Collection Krasske in Naturkundemuseum im Ottoneum (KASSEL), Kassel, Germany.

Type locality. Demerara River, Guyana.

Valves linear-lanceolate; narrowly rounded apices; length: 48-60 $\mu \mathrm{m}$, breadth: 7-9 $\mu \mathrm{m}$, length/breadth ratio: 5.97-7.92; axial area lanceolate; central area rectangular, reaching the valvae margins; raphe lateral, proximal raphe fissures unilaterally deflected; striae short, radiate, becoming convergent towards to the ends of the valves; striae: $10-11 / 10 \mu \mathrm{m}$.

Material examined. Brazil, State of Goiás, Britânia, Lago dos Tigres, Baía das Perdidas, periphyton, Nogueira et al. Site 2, 13/07/2004, ISN939 (UFG 29945); Água Limpa River, plankton, Nogueira et al. Site 1, 09/09/2004, ISN985 (UFG 29907).

Distribution. Brazil, State of Amazonas, Lago Calado; State of Pará, Tapajós River (Metzeltin \& Lange-Bertalot 1998); Pioneer citation for the State of Goiás.

Remarks. Pinnularia certa is very similar to P. microstauron var. brasiliensis Krammer \& Metzeltin (see below), including metric characteristics as recorded in their original descriptions (Metzeltin \& Lange-Bertalot 1998). However, P. certa presents wider central margin reaching the margins than in $P$. microstauron var. brasiliensis. Moreover, $P$. microstauron var. brasiliensis has straight and parallel margins while in $P$. certa the margins are slightly convex.

The outline of the individuals found in Lago dos Tigres was similar to $P$. certa. However, some of these representatives were longer (48-60 $\mu \mathrm{m}$ against $43-54 \mu \mathrm{m})$ and or narrower $(7-9 \mu \mathrm{m}$ against $8.4-9.4 \mu \mathrm{m}$ ) than the type population characterized by Metzeltin \& Lange-Bertalot (1998).

\section{Pinnularia cf. acuminata}

W.Sm., Syn. British. Diat., vol. 1, p. 55, Fig. 18: 164, 1853. (Figures 3, 4)

Lectotype. Preparation IX-38-A7 VH, Collection Van Heurck in the Dr. Henri Van Heurck Museum (AWH), Antwerp, Belgium, designated by Krammer (2000, p. 159).

Isolectotype. Preparation 20600 BM, Collection Natural History Museum in the Natural History Museum (BM), London, England, designated by Krammer (2000, p. 159).

Type locality. Premmay Peat, Aberdeenshire, Scotland.

Valves linear, sometimes with slightly concave margins at the middle part; cuneate apices; length: $45-61 \mu \mathrm{m}$, breadth: 10.5-12 $\mu \mathrm{m}$, length/breadth ratio: 4.01-5.79; axial area wide, linear-lanceolate, reaching $\frac{1}{2}$ of the breadth of the valvae; central area with thin fascia, reaching the valves margins; raphe lateral, proximal ends slightly curved to the same side; striae short, parallel, becoming slightly convergent towards to the ends of the valves; striae: $10-11 / 10 \mu \mathrm{m}$.

Material examined. Brazil, State of Goiás, Britânia, Lago dos Tigres, Baía das Perdidas, periphyton, Nogueira et al., Site 2,
13/07/2004, ISN939 (UFG 29945); Água Limpa River, plankton, Nogueira et al., Site 1, 09/09/2004, ISN985 (UFG 29907); Lago dos Tigres, plankton, Nogueira et al., Site 10, 18/ 07/2008, P10M07, ISN1235 (UFG 43645), Site 01, 15/11/2008, P01M11, ISN1278 (UFG 43688).

Distribution. Brazil, State of Amazonas, Negro River (Metzeltin \& Lange-Bertalot 2007); State of Paraná, Guaraguaçu River (Tremarin et al. 2010); Pioneer citation for the State of Goiás.

Remarks. $P$. acuminata and $P$. inconstans Mayer presents similar outlines, but the first one has acuminate apices, wider axial area and an inconspicuous to small fascia (Krammer 2000, figures 144: 7-9). On the other hand, the neotype specimens of $P$. inconstans have more rounded ends of the valvae, narrower axial area, and a conspicuous fascia (Krammer 2000, figures 143:3-5, 11, 12). Such similarities lead to confusions concerning the identification of the two taxa in some studies of diatom flora.

Pinnularia inconstans sensu Krammer (2000, Fig 143: 10), from Demerara River, Guyana, and $P$. inconstans sensu Tremarin et al. (2010), from the State of Paraná, Brazil, for example, present more similar characteristics to the complex $P$. acuminata, specially $P$. acuminata var. guyanensis Metzeltin \& Lange-Bert. (Metzeltin \& Lange-Bertalot 2007). This taxa differs from the nominated variety because breather $(9.5-10$ against $12-16 \mu \mathrm{m})$ and smaller valves (36-42 against 40-84 $\mu \mathrm{m})$, and higher density of striae (10-11 against 8-10 striae per $10 \mu \mathrm{m})($ Metzeltin \& Lange-Bertalot 2007).

The representatives of Pinnularia cf. acuminata from Lago dos Tigres were similar to $P$. inconstans sensu Krammer (2000, Fig 143: 10) and P. inconstans sensu Tremarin et al. (2010), and, concomitantly, to $P$. acuminata var. guyanensis, but presented breadth values intergrading with those of the nominated variety. The densities of striae of the specimens from Lago dos Tigres were slightly higher than $P$. acuminata var. acuminata but agreed with $P$. acuminata var. guyanensis. However, Pinnularia cf. acuminata presented a wider axial area and more cuneated valvar ends than the type of $P$. acuminata.

The outline, including valvar ends, and axial area of Pinnularia cf. acuminata was similar to $P$. acuminata var. novaezealandica Krammer sensu Tremarin et al. (2010). However, the representatives found in Lago dos Tigres were smaller (45-61 $\mu \mathrm{m}$ against 86.1-133.3 $\mu \mathrm{m})$, and narrower $(10.5-12 \mu \mathrm{m}$ against $13.3-14.1 \mu \mathrm{m})$ than the specimens in Tremarin et al. (2010). Similarly to our representatives, $P$. acuminata var. novaezealandica sensu Tremarin et al. (2010, figure 9) differs from the type specimens of this taxon as illustrated by Krammer (2000, figures 148: 1-5) because presents wider axial area and more cuneated valvar ends, although agreed partially with $P$. acuminata var. novaezealandica [novazealandica] sensu Metzeltin \& LangeBertalot (2007, Figures 262: 1-3).

The individuals from Lago dos Tigres present outline to P. subacuminata Krammer \& Metzeltin, but still here the axial area of Pinnularia cf. acuminata was slightly narrower, the valvar ends were cuneate instead cuneate-rounded, as observed in P. subacuminata. Moreover, P. subacuminata is larger (45-61 $\mu \mathrm{m}$ against $95-115 \mu \mathrm{m})$ than the specimens from Lago dos Tigres.

Compared to P. angustivalva Krammer \& Metzeltin, Рinnularia cf. acuminata present axial area slightly wider, smaller central area, margins slightly convex, and valvar ends cunead-obtuse against cuneate in the representatives os Lago dos Tigres. Some 
representatives of Pinnularia $\mathrm{cf}$. acuminata were larger $(38-54 \mu \mathrm{m}$ against $45-61 \mu \mathrm{m})$ and all were wider $(6.7-8 \mu \mathrm{m}$ against $10.5-12$ $\mu \mathrm{m})$, and less densely striated $(13-14 / 10 \mu \mathrm{m}$ against $10-11 / 10 \mu \mathrm{m})$ than $P$. angustivalva.

Other similar species is $P$. instabiliformis Krammer \& Metzeltin, which also present wide axial area but the valvar ends cuneate-subrostrate (Metzeltin \& Lange-Bertalot 1998, figures 8-11) or cuneate- rounded (Metzeltin \& Lange-Bertalot 1998, Figures 8-12) comparing to the simply cuneate valvar ends of Pinnularia cf. acuminata. Some representatives of Pinnularia cf. acuminata were larger $(36-52 \mu \mathrm{m}$ against $45-61 \mu \mathrm{m})$ or slightly wider $(10.4-11.7 \mu \mathrm{m}$ against $10.5-12 \mu \mathrm{m})$ than $P$. instabiliformis.

Since Lago dos Tigres presented intermediate specimens between several taxa compared here, we identified the taxon as Pinnularia cf. acuminata, the oldest epithet and the most similar taxon of this complex. More accurate studies should be carried out in order to verify the limits and circumscription among the taxa of this complex.

\section{Pinnularia divergens var. mesoleptiformis}

Metzeltin e Lange-Bert., Iconogr. Diatomol, vol. 5, p. 170, Figs. 173: 1-4, 1998. (Figure 24)

Holotype. Preparation AmS-521, Collection Lange-Bertalot in Botanisches Institüt der J. W. Goethe Universität, Frankfurt/ Main, Germany.

Type locality. Kaieteur-Falss, Potaro River, Guyana.

Valves lanceolate, triundulate margins; rounded apices; length: 64.24-72.81 $\mu \mathrm{m}$, breadth: 12.04-13.78 $\mu \mathrm{m}$, length/ breadth ratio: $4.82-5.25$; axial area linear; central area rhomboid, with fascia reaching the margins; raphe lateral, terminal fissures bayonet-shapes, proximal fissures curved to the same side; striae radiated, becoming convergent towards to the ends of the valves; striae: $10-11 / 10 \mu \mathrm{m}$.

Material examined. Brazil, State of Goiás, Britânia, Lago dos Tigres, plankton, Nogueira et al. Site 5, 04/07/2004, ISN878 (UFG 29867); Site 4, 04/06/2004, ISN877 (UFG 29866); Site 11, 17/10/2004, ISN1033 (UFG 29928);

Distribution. Brazil, State of Paraná, Guaraguaçu River (Tremarin et al. 2009, 2010); State of São Paulo, São Paulo, Parque Estadual das Fontes do Ipiranga (Rocha \& Bicudo 2008); Pioneer citation for the State of Goiás.

Remarks. The specimens from Lago dos Tigres agreed with the description provided by Metzeltin \& Lange-Bertalot (1998). They also were similar but smaller than those illustrated by Rocha \& Bicudo (2008) and Tremarin et al. (2010).

\section{Pinnularia hudsonii}

Metzeltin, Lange-Bert. \& García-Rodríguez, Iconogr. Diatomol., vol. 15, p. 156, Figs 177:1-11, 2005. (Figures 5, 6) Holotype. Preparation Uru 2002-2, Collection Lange-Bertalot in Botanisches Institüt der J. W. Goethe Universität, Frankfurt/Main, Germany.

Type locality. Rio de La Plata, near Colonia Del Sacramento, Uruguay.

Valves lanceolate; rostrate-cuneate apices; length: 61.81$67.64 \mu \mathrm{m}$, breadth: $10-11 \mu \mathrm{m}$, length/breadth ratio: 5.6-6.6; axial area lanceolate, broad; central area with fascia, reaching the valvae margins; raphe lateral, terminal ends hooked, proximal ends slightly curved in the same direction.; striae radiate, becoming convergent towards to the ends of the valves; striae: $10-11 / 10 \mu \mathrm{m}$.

Material examined. Brazil, Goiás, Britânia, Lago dos Tigres, plankton, Nogueira et al., Site 8, 13/07/2004, ISN904 (UFG 29887), Site 7, 17/07/2008, P07M07, ISN1232 (UFG 43642); Site 10, 18/07/2008, P10M07, ISN1235 (UFG 43645), Site 11, 20/10/2008, P11M10, ISN1275 (UFG 43685).

Distribution. Brazil, State of Paraná, Guaraguaçu River (Tremarin et al. 2010); Salto Amazonas River (Santos et al. 2011); Pioneer citation for the State of Goiás.

Remarks. The specimens from Lago dos Tigres agreed with the type material, except by the ends which are slight cuneate in the material from Britânia. Similar ends were observed also in the material from Guaraguaçu River (Tremarin et al. 2010, figure 34 ), what suggest this characteristic as a possible morphological variation into the species.

\section{Pinnularia instabilis}

(A. Schmidt) Metzeltin in Metzeltin \& Lange-Bertalot, Iconogr. Diatomol., vol. 5, p. 178, Figs. 194: 1-3, Figs. 205: 4-5, 1998. (Figure 7)

Basionym. Navicula instabilis A.Schmidt, A. Schmidt's Atlas, Figures 43: 38-40, non Figures 43: 35-37, 1875.

Lectotype (designated here). One figure in Schmidt (1875, Figure 43: 39 ) (Figure 7)

Lectotype locality. Demerara River, Guyana.

Valves lanceolate, wide; acuminate or attenuated-peaked apices; length: $53.71-83.11 \mu \mathrm{m}$, breadth: $17.7-21.3 \mu \mathrm{m}$, length/ breadth ratio: $3.03-4.54$; axial area lanceolate, broad, reaching $\frac{1}{2}$ of the breadth of the valvae; raphe filiform to slightly lateral, terminal ends hooked and proximal ends curved to the same direction; striae parallel becoming slightly radiate towards to the ends of the valves; striae: $10 / 10 \mu \mathrm{m}$.

Material examined. Brazil, State of Goiás. Britânia, Lago dos Tigres, plankton, Nogueira et al., Site 2, 13/07/2004, ISN898 (UFG 29880); Site 4, 04/06/2004, ISN877 (UFG 29866), Site 7, 17/07/2008, P07M07, ISN1232 (UFG 43642); Site 01, 16/09/ 2008, P01M09, ISN1252 (UFG 43662); Site 02, 16/09/2008, P02M09, ISN1253 (UFG 43663); Site 11, 20/10/2008, P11M10, ISN1275 (UFG 43685), periphyton, Site 2, 09/09/2004 ISN1014 (UFG 29953).

Distribution. Brazil, State of Amazonas, Tupé Lake (Pereira et al. 2013); Pioneer citation for the State of Goiás.

Remarks. In its original proposal, Schmidt (1875, Figures 43: 35-40) illustrated $P$. instabilis. In the legend of these illustrations, he (Schmidt 1875) highlighted his figures 43: 37 and 43: 39 with the acronym "typ.", indicating there and in other parts of his study, the typical representatives of respective taxa. Based on the outline and measures of these illustrations, Metzeltin in Metzeltin \& Lange-Bertalot (1998, p. 178) considered the set of Schmidt's specimens of Figures 43: $35-37$ as $P$. subacuminata Krammer \& Metzeltin and regarded the name $P$. instabilis to Schmidt's specimens from figures 43: 38-40. Since the preferences of Schmidt (1875, figures 43: 37, 39) proven to be ambiguous and that, among them, only remain the figure 43: 39 as the original sense, we designated it as lectotype. Complementarily, we designated as epitype one specimen in preparation from a sample from Demerara River, Guyana, in Collection Hustedt called "A.S." (A.Schmidt), which the specimens found in Lago dos Tigres agreed. 


\section{Pinnularia latarea}

Krammer, Diatoms of Europe, vol. 1, p. 110, 224, Figs. 80: 1-6, 84: 13-15, 2000. (Figure 18)

Holotype. Preparations 89, 101 and 102 IOS, Collection Schimanski in Institut für Oberflächenanalyse, Meerbusch, Germany.

Type locality. Mönau-Weiher, near Erlangen, Bavaria, Germany.

Valves linear, with slightly undulated margins; capitates apices; length: 45.69-62.6 $\mu \mathrm{m}$, breadth: $7.91-10.38 \mu \mathrm{m}$, length/ breadth ratio: 5.77-6.03; axial area narrowly lanceolate, central area expanded, with fascia until reaching the margins; raphe filiform to slightly lateral; striae radiated, becoming convergent towards to the ends of the valves; striae: $11-12 / 10 \mu \mathrm{m}$.

Material examined. Brazil, State of Goiás. Britânia, Lago dos Tigres, plankton, Nogueira et al. Site 1, 16/09/2008, P01M09 (UFG 43662), Site 1, 19/08/2008, P01M08 (UFG 43649); Site 07, 19/08/2008, P07M08 (UFG 43655); Site 1, 19/10/2008, P01M10 (UFG 43675); Site 5, 04/07/2004, ISN878 (UFG 29867); Água Limpa River, plankton, Nogueira et al. Site 1, plankton, 09/09/2004, ISN985 (UFG 29907).

Distribution. Brazil, State of São Paulo, São Carlos, Monjolinho River (Souza \& Senna 2009); State of Paraná, Maringá, Nazaré Stream (Moresco et al. 2011); Pioneer citation for the State of Goiás;

Remarks. The specimens found in Lago dos Tigres agreed with the original description provided by Krammer (2000), except by some representatives more densely striated (11-12/10 $\mu \mathrm{m}$ against $8-10 / 10 \mu \mathrm{m})$. However, the specimens observed in this study presented more linear outlines than the type specimens (Krammer 2000, figures 84: 13-15). The measures and outlines of the Lago dos Tigres material were also similar to that provided by Metzeltin et al. (2005, figures 168:1-14), from Uruguay material, and to that found by Souza \& Senna (2009), in São Paulo, including the slight undulation observed in the margins.

Two representatives of $P$. latarea found by Moresco et al. (2011, figures 40,41) presented similar outlines the type specimens provided by Krammer (2000). A third individual (Moresco et al, 2011, figure 42), however, had outline linear similarly to the individuals observed in Lago dos Tigres.

\section{Pinnularia mayeri}

Krammer, Biblioth. Diatomol., vol. 26, p. 115, Figs. 42: 1-4, 1992. (Figures 8, 9)

Holotype. Preparation 912 MR, Collection Mayer in Regensburgische Botanische Gesellschaft (REG), Regensburg, Germany.

Type locality. Outlet of a fish pond, near Hozheim (Oberpfalz near Kladorf), Bavaria, Germany.

Valves linear-lanceolate; capitate apices; length: $52 \mu \mathrm{m}$, breadth: $7 \mu \mathrm{m}$, length/breadth apices: 7.42; axial area linearlanceolate, central area rhomboid, with fascia reaching the margins; raphe filiform, terminal fissures hooked and proximal fissures curved to the same side; striae radiate, becoming convergent toward to the ends of the valves; striae: $10-11 / 10 \mu \mathrm{m}$ Material examined. Brazil, State of Goiás, Britânia, Lago dos Tigres, plankton, Nogueira et al. Site 4, 04/06/2004, ISN877 (UFG 29866); Site 11, 06/11/2004, ISN1113 (UFG 29939), Lago dos Tigres, Baía das Perdidas, periphyton, Nogueira et al., Site 2, 13/07/2004, ISN936 (UFG 29942), 13/07/2004, ISN939 (UFG 29945);
Distribution. Brazil, State of São Paulo, São Paulo, Parque Estadual das Fontes do Ipiranga (Rocha \& Bicudo 2008); Pioneer citation for the State of Goiás.

Remarks. Pinnularia mayeri found in Lago dos Tigres agreed with those found in São Paulo by Rocha \& Bicudo (2008) and with the original description provided by Krammer (2000). However, the individuals from type material presented outline more lanceolate than our specimens (see Krammer 2000, Figures 96: 9-13).

The outline and measures of the specimens from Lago dos Tigres are also similar to Pinnularia pisciculus var. angusta Metzeltin \& Krammer in Metzeltin \& Lange-Bertalot (1998, figures 175: 6-12, 194: 5, 6), and those illustrated in Metzeltin \& Lange-Bertalot (2007, figures 267: 8-12, 273: 1-8), except by the more prominent valvar ends, as well as in $P$. mayeri. $P$. pisciculus var. angusta also presents specimens with outline more linear, such as in the Lago dos Tigres material, to lanceolate specimens such as in $P$. mayeri. This variance can suggest a possible conspecificity of these two taxa.

\section{Pinnularia meridiana var. meridiana}

Metzeltin \& Krammer in Metzeltin \& Lange-Bertalot, Iconogr. Diatomol., vol. 5, p. 180, Figs 181: 1, 2, 4, 5, 1998. (Figures 10, 11)

Holotype. Preparation AmS-305, Colletion Lange-Bertalot in Botanisches Institüt der J. W. Goethe Universität, Frankfurt/ Main, Germany.

Type locality. Stream in Porto Alegre, State of Rio Grande do Sul, Brazil.

Valves linear, with bi-undulate margins; broad cuneaterounded apices; length: 43.81-62.79 $\mu \mathrm{m}$, breadth: $8.22-11.37$ $\mu \mathrm{m}$, length/breadth ratio: 4.01-6.16; axial area rhomboid; central area wide, rhomboid, with fascia not reaching the margins; raphe lateral, terminal fissures hooked, with helictoglossa present (Figure 11), proximal ends curved to the same side; striae parallel to slightly radiated, becoming convergent towards to the ends of the valves; striae: $11 / 10 \mu \mathrm{m}$.

Material examined. Brazil, State of Goiás, Britânia, Lago dos Tigres, plankton, Nogueira et al. Site 1, 17/07/2008, P01M07, ISN1226 (UFG 43636), Site 10, 18/07/2008, P10M07, ISN1235 (UFG 43645); Site 4, 11/12/2008, P04M12, ISN1294 (UFG 43704); Site 5, 04/07/2004, ISN878 (UFG 29867); Site 3, 19/08/ 2004, ISN945 (UFG 29895); Site 5, 09/09/2004, ISN989 (UFG 29911); Vermelho River, plankton, Nogueira et al. Site 11, 05/ 07/2004, ISN881 (UFG 29870); Site 11, 17/10/2004, ISN1033 (UFG 29928); Água Limpa River, plankton, Nogueira et al. Site 1, 14/07/2004, ISN908 (UFG 29891).

Distribution. Brazil, State of Goiás, Formosa, Paranã River, site 15; Salobro River, site 16; Crixás River, site 17; Paranã River, site 29; Nova Roma, Forquilha Stream, site 7 (Souza \& Oliveira 2007, da Silva et al. 2011); State of São Paulo, São Carlos, Monjolinho River (Souza \& Senna 2009); State of Paraná, Matinhos, Guaraguaçu River; Pontal do Paraná, Guaraguaçu River; General Carneiro, Salto do Amazonas River; Lagoa da Fazenda São Pedro; Araucárias Reservoir; Neno Stream (Tremarin et al. 2009); State of Rio Grande do Sul, Porto Alegre (from the original material in Metzeltin \& Lange-Bertalot 1998).

\section{Pinnularia microstauron var. brasiliensis}

Krammer \& Metzeltin, Iconogr. Diatomol, vol. 5, p. 181, Figs. 179: 9-15, 1998. (Figures 13-15) 
Holotype. Preparation AmS-562, Colletion Lange-Bertalot in Botanisches Institüt der J. W. Goethe Universität, Frankfurt/ Main, Germany.

Type locality. Tapajós River, State of Amazonas, Brazil.

Valves linear, with bi-undulated margins; cuneate-rostrate apices; length: 41.14-45.79 $\mu \mathrm{m}$, breadth: 7.22-8.6 $\mu \mathrm{m}$, length/ breadth ratio: 5.37-6.57; axial area rhomboide; central area rhomboidal, with fascia reaching the margins; raphe lateral, terminal fissures hooked, proximal fissures curved to the same side; striae short, parallel to radiate, becoming convergent towards to the ends of the valves; striae: $10-11 / 10 \mu \mathrm{m}$

Material examined. Brazil. Goiás. Britânia. Lago dos Tigres, plankton, Nogueira et al. Site 1, 16/09/2008, P01M09, ISN1252 (UFG 43662), Site 01, 15/11/2008, P01M11, ISN1278 (UFG 43688), Site 5, 19/08/2008, P05M08, ISN1243 (UFG 43653).

Distribution. Brazil, State of Pará, Tapajós River (from the original material in Metzeltin \& Lange-Bertalot 1998); Pioneer citation for the State of Goiás.

Remarks. See comments above, in P. certa.

\section{Pinnularia microstauron var. rostrata}

Krammer, Diatoms of Europe, vol. 1, p. 74, 217, Figs. 51: 8-18, 2000. (Figures 16, 17)

Holotype. Preparation 18B, Collection Krammer in AlfredWegener-Institut für Polar- und Meeresforschung (BRM), Bremerhaven, Germany.

Type locality. Hesepertywist near Nordhorn, Emsland, Germany.

Valves linear; rostrate apices; length: $35.2-36.49 \mu \mathrm{m}$, breadth 6.23-7.32 $\mu \mathrm{m}$, length/breadth ratio: 4.89-5.41; axial area rhomboid; central area romphoid, with fascia reaching the margins; raphe filifom, terminal fissures hooked, proximal fissures curved to the same side; striae radiate, becoming convergent towards to the ends of the valves; striae: $10-13 / 10 \mu \mathrm{m}$

Material examined. Brazil, State of Goiás, Britânia, Lago dos Tigres, plankton, Nogueira et al. Site 1, 19/08/2008, P01M08, ISN1239 (UFG 43649).

Distribution. Pioneer citation for the country.

Remarks. Pinnularia microstauron var. rostrata distinguish from the nominate variety because is narrower $(6-7 \mu \mathrm{m}$ against $10-12.4 \mu \mathrm{m}$ ) and presents rostrate apices (Krammer 2000). This taxon is somewhat similar to P. microstauron var. brasiliensis considering the dimensions, the valvar outline and axial and central areas, but presents clear differences respect to the shape of the apices, which is narrower in the variety brasiliensis than in the variety rostrata (Figures 13-17).

The specimens observed in Lago dos Tigres were very similar to the material type of $P$. microstauron var. rostrata described by Krammer (2000), with some individuals more densely striated than the type population $(10-13 / 10 \mu \mathrm{m}$ against $10-11 / 10 \mu \mathrm{m})$. The representatives from Lago dos Tigres were also similar to the specimens illustrated by Metzeltin et al. (2005, Figures 164: 7, 8, 23), which, however, presented less prominent apices compared to those.

The specimens of the Lago dos Tigres were also similar to Pinnularia cf. microstauron var. rostrata observed by Canani et al. (2011), from Minas Gerais. Nevertheless, the only one specimen recorded by Canani et al. (2011) was larger than the type of $P$. microstauron var. rostrata and our specimens $(9.8 \mu \mathrm{m}$ against $6-8 \mu \mathrm{m})$, and it can belong to another taxa, closed to this group.

\section{Pinnularia perumbrosa}

Metzeltin \& Lange-Bert., Iconogr. Diatomol, v. 18, p. 215, Figs. 269: 1, 2, 270: 1-3, 2007. (Figure 20)

Basionym. Pinnularia umbrosa var. tropica Metzeltin \& Krammer in Metzeltin \& Lange-Bertalot, Iconogr. Diatomol, v. 5, p. 194, Figs. 182: 7-9, 1998.

Holotype. Preparation AmS-597, Collection Lange-Bertalot in Botanisches Institüt der J. W. Goethe Universität, Frankfurt/ Main, Germany.

Type locality. State of Amazonas, Brazil.

Valves linear, triundulate and inflamade at the middle part; broad capitates apices; length: 105.49-148.22 $\mu \mathrm{m}$, breadth: 11.13-17.88 $\mu \mathrm{m}$, length/breadth ratio: 7.78-8.78; axial area linear-lanceolate, reaching $1 / 3$ of the breadth of the valvae; central area rhomboid, with fascia reaching the margins; raphe lateral, terminal fissures sickle-shaped, proximal fissures slightly curved to the same side; striae radiate, becoming convergent towards the ends of the valves; striae: $10-12 / 10 \mu \mathrm{m}$. Material examined. Brazil, State of Goiás, Britânia, Lago dos Tigres, plankton, Nogueira et al. Site 05, 17/07/2008, P05M07, ISN1230 (UFG 43640); Site 01, 19/10/2008, P01M10, ISN1265 (UFG 43675).

Distribution. Brazil, State of Amazonas, Calado Lake (from the original material in Metzeltin \& Lange-Bertalot 1998, 2007); Negro River (Pereira et al. 2012); Pioneer citation for the State of Goiás.

Remarks. Pinnularia umbrosa var. tropica was initially described from fossil material. The type material presented 87-100 $\mu \mathrm{m}$ length, 13.4-14.7 $\mu \mathrm{m}$ breadth, 6.59-7.3 length/ breadth ratio, and 10-12 striae in $10 \mu \mathrm{m}$ (Metzeltin \& LangeBertalot 1998). Metzeltin \& Lange-Bertalot (2007) considered the morphometric differences between $P$. umbrosa var. tropica and the nominated variety as enough to rising that taxon at specific level, under the new name $P$. perumbrosa, since the combination with epithet tropica would not be possible into the genus Pinnularia. In this study, Metzeltin \& Lange-Bertalot (2007) identified other fossil specimens from Amazonas as P. perumbrosa. These specimens presented 98.46-113.0 $\mu \mathrm{m}$ length, 12.31-13.85 $\mu \mathrm{m}$ breadth, length/ breadth ratio 7.33-8.65, amplifying the metric characteristics of this species. Moreover, both group of specimens illustrated by Metzeltin \& LangeBertalot (1998) and Metzeltin \& Lange-Bertalot (2007) have margins conspicuously undulated and breath values that decrease from the middle part towards the ends of the valvae.

Live specimens of $P$. perumbrosa were recorded by Pereira et al. (2012). These specimens presented similar outlines compared to the Metzeltin \& Lange-Bertalot's (1998, 2007) material. Although the $P$. perumbrosa sensu Pereira et al. (2012) was larger than the type population, this agreed with those specimens illustrated by Metzeltin \& Lange-Bertalot (2007).

Metzeltin \& Lange-Bertalot (2007) also described $P$. diandae Metzeltin \& Lange-Bert. with undulated margins and similar structure to $P$. perumbrosa, highlighting metric characters as diacritic. Pinnularia diandae reach $260 \mu \mathrm{m}$ length, and about $20 \mu \mathrm{m}$ breadth.

The material observed in Lago dos Tigres presented larger specimens than any material of $P$. perumbrosa but it could metrically agree with $P$. diandae. Nevertheless, the smallest length values of our material interbreed with measures of $P$. perumbrosa (Metzeltin \& Lange-Bertalot 2007, Pereira et al. 2012). Moreover, our material presented also a decrease of the breadth values from 
the middle part towards to the ends of the valvae, similarly to $P$. perumbrosa, what is not observed in $P$. diandae.

\section{Pinnularia rostratissima f. subundulata}

Hust., Int. Rev. Hydrobiol., vol. 50, p. 397, Fig. 27, 1965. (Figure 22)

Holotype. Specimen marked in preparation P5/17, Finder 582.4-7, Collection Hustedt in Alfred-Wegener-Institut für Polar- und Meeresforschung (BRM), Bremerhaven, Germany (Simonsen 1987, Figure 754: 2).

Type locality. Tapajoz River, State of Pará, Brazil.

Valves rhomboid-lanceolate, inflated at the middle part and slightly undulated margins; prominent capitate apices; length: 81.85-91.22 $\mu \mathrm{m}$, breadth: 12.47-15.43 $\mu \mathrm{m}$, length/breadth ratio: 5.83-6.53; axial area broad, rhomboid; central area rhomboid, reaching the margins; raphe lateral, terminal fissures hooked, proximal fissures curved to the same side; striae short, radiate, becoming convergent towards the ends of the valves; striae: $8-10 / 10 \mu \mathrm{m}$.

Material examined. Brazil, State of Goiás, Britânia, Lago dos Tigres, plankton, Nogueira et al. Site 5, 04/07/2004, ISN878 (UFG 29867); Site 4, 04/06/2004, ISN877 (UFG 29866); Site 1, 09/09/2004, ISN985 (UFG 29907); Site 6, 16/10/2004, ISN1028 (UFG 29923); Site 8, 16/10/2004, ISN1030 (UFG 29925), Lago dos Tigres, Baía das Perdidas, periphyton, Nogueira et al. Site 2, 13/07/2004, ISN939 (UFG 29945).

Distribution. Brazil, State of Amazonas (Metzeltin \& LangeBertalot 1998); State of Pará, Tapajós River, Arapiuns River (Hustedt 1965, Simonsen 1987); Pioneer citation for the State of Goiás.

Remarks. see remarks in $P$. rostratissima var. rostratissima.

\section{Pinnularia rostratissima var. rostratissima}

Hustedt, Int. Rev. Hydrobiol., vol. 50, p. 397, Fig. 26, 1965. (Figure 21)

Holotype. Specimen marked in preparation P5/16, Finder 354.16, Collection Hustedt in Alfred-Wegener-Institüt für Polar- und Meeresforschung (BRM), Bremerhaven, Germany (Simonsen 1987, Figure 754: 1).

Type locality. Tapajós River, State of Pará, Brazil.

Valves fusiform-lanceolate, strongly inflated at the middle part; capitates apices; length: $81.27-88.12 \mu \mathrm{m}$, breadth: $13.78-$ 15.63; length/breadth ratio: 5.63-5.89; axial area broad, lanceolate; central area lanceolate, with fascia reaching the margins; raphe lateral, terminal fissures hooked, proximal fissures curved to the same side; striae short, radiate, becoming convergent towards to the ends of the valves; striae: $10-11 / 10 \mu \mathrm{m}$. Material examined. Brazil, State of Goiás, Britânia, Lago dos Tigres, plankton, Nogueira et al. Site 11, 20/10/2008, P11M10, ISN1275 (UFG 43685).

Distribution. Brazil, State of Amazonas, Jaú River (DíazCastro et al. 2003); State of Pará, Tapajós River, Arapiuns River (Hustedt 1965, Simonsen 1987, Metzeltin \& LangeBertalot 1998); Pioneer citation for the State.

Remarks. The original material of $P$. rostratissima presented length $78-93 \mu \mathrm{m}, 11-14 \mu \mathrm{m}$ breadth and $10-11$ striae in $10 \mu \mathrm{m}$ (Hustedt 1965). Hustedt (1965) also described P. rostradissima f. subundulata Hust. which differed from nominate forma from the ends of the valve and slight undulations on the margins. Moreover, the outline of the forma subundulata is some rhomboidal whereas in the forma rostratissima is some fusiform.

Simonsen (1987) illustrated the type of $P$. rostratissima which, according to Metzeltin \& Lange-Bertalot (1998) presented length 94 $\mu \mathrm{m}$, breath 13.4, 9.5-10 striae in $10 \mu \mathrm{m}$. Based on these estimative, Metzeltin \& Lange-Bertalot (1998) proposed P. rostratissima var. parva Metzeltin \& Lange-Bert. and P. rostratissima var. ventricosa. Metzeltin \& Lange-Bert. According these authors, the variety parva would have narrower specimens than the nominate variety (10-11 $\mu \mathrm{m})$, while the variety ventricosa would be broader specimens (14-17.5 $\mu \mathrm{m})$. However, Metzeltin \& Lange-Bertalot (1998) did not take into account the range of measures of specimens in the population characterized by Hustedt (1965), which are intersected with the breath values of the varieties parva and ventricosa. Despite this, the specimens of $P$. rostratissima var. parva described by Metzeltin \& Lange-Bertalot (1998) are smaller than the nominate variety $(59-63 \mu \mathrm{m}$ against $78-94 \mu \mathrm{m}) . \quad P$. rostratissima var. ventricosa may be similarly distinguished from the nominated variety (54-74 $\mu \mathrm{m}$ against 78-94 $\mu \mathrm{m})$. Moreover, this taxon presents specimens visually more inflated what can be numerically proved by lower length/breadth values [3.86-4.41 (5.28) instead $\geq$ 5.57].

The specimens of $P$. rostratissima var. rostratissima found in Lago dos Tigres are similar to the outline of the type specimen illustrated by Simonsen (1987, Figure 754: 1). The breath values of these specimens intergrading with the values of $P$. rostratissima var. rostratissima, as described by Hustedt (1965), and P. rostratissima var. ventricosa. But they would not match with $P$. rostratissima var. ventricosa, because it was larger and not so inflated as the variety ventricosa (i.e., length/breadth value higher than 5.57).

\section{Pinnularia rostratissima var. ventricosa}

Metzeltin e Lange-Bert., Iconogr. Diatomol, vol. 5, p. 186, Figs. 169: 1-5, 203: 1, 4, 1998. (Figure 23)

Holotype. Preparation AmS-526, Collection Lange-Bertalot in Botanisches Institüt der J. W. Goethe Universität, Frankfurt/ Main, Germany.

Type locality. Essequibo-River, Guyana.

Valves fusiform-lanceolate, inflated at the middle part; capitate apices; length: 70; breadth: 15, length/breadth ratio: 4.66; axial area fusiform, central area indistinct of the axial area, with fascia reaching the margins; raphe lateral, terminal fissures hooked, proximal fissures curved to the same side; striae short, slightly radiated, becoming convergent towards the ends of the valves; striae: $12 / 10 \mu \mathrm{m}$.

Material examined. Brazil, State of Goiás, Britânia, Lago dos Tigres, plankton, Nogueira et al., Site 8, 13/07/2004, ISN904 (UFG 29887); Vermelho River, plankton, Nogueira et al. Site 11, 05/06/ 2004, ISN881 (UFG 29870), Site 10, 18/07/2008, P10M07, ISN1235 (UFG 43645); Site 01，16/09/2008，P01M09，ISN1252 (UFG 43662); Site 11, 20/10/2008, P11M10, ISN1275 (UFG 43685).

Distribution. Brazil, State of Amazonas (Metzeltin \& LangeBertalot 1998), Negro River (Metzeltin \& Lange-Bertalot 2007, Pereira et al. 2012); Tupé Lake (Pereira et al. 2013); Pioneer citation for the State of Goiás.

Remarks. see remarks in $P$. rostratissima var. rostratissima.

\section{Pinnularia rumrichiae}

Krammer, Diatoms of Europe, vol. 1, p. 110, 223, Figs. 51: 8-18, 2000. (Figure 26) 
Holotype. Preparation Eu-SF 117, Collection Lange-Bertalot in Botanisches Institüt der J. W. Goethe Universität, Frankfurt/Main, Germany.

Type locality. Julma, Ölkky near Kuusamo, Finland.

Valves linear; capitates apices, with very narrow necks; length: $38.17-43.24 \mu \mathrm{m}$, breadth: $7.12-8.01 \mu \mathrm{m}$, length/breadth ratio: 4.81-5.92; axial area lanceolate; central area rhomboid, with fascia reaching the margins; raphe filiform to slightly lateral, terminal fissures hooked, proximal fissures curved to the same side; striae radiated, becoming convergent towards to the ends of the valves; striae: $10-12 / 10 \mu \mathrm{m}$.

Material examined. Brazil, State of Goiás, Britânia, Lago dos Tigres, plankton, Nogueira et al. Site 5, 04/07/2004, ISN878 (UFG 29867); Site 4, 04/06/2004, ISN877 (UFG 29866); Site 8, 16/10/2004, ISN1030 (UFG 29925), Site 6, 09/09/2004, ISN989 (UFG 29911); Vermelho River, plankton, Nogueira et al. Site 11, 14/07/2004, ISN907 (UFG 29890); Site 4, 19/08/2008, P04M08, ISN1242 (UFG 43652), Site 01, 16/09/2008, P01M09, ISN1252 (UFG 43662), Site 1, 19/10/2008, P01M10, ISN1265 (UFG 43675), Site 9, 19/08/2008, P09M10, ISN1273 (UFG 43683), Site 01, 15/11/2008, P01M11, ISN1278 (UFG 43688).

Distribution. Brazil, State of Paraná, Guaraguaçu River (Tremarin et al. 2010); Pioneer citation for the State of Goiás.

Remarks. The specimens from Lago dos Tigres agreed with the original description of $P$. rumrichiae, except in cases that some specimens presented lower length/breadth ratio (4.81-5.85 against 5.6-6.8). Our material was also similar to specimens of $P$. rumrichiae observed by Tremarin et al. (2010) [cited as $P$. "rumrichae" but correted in this study according to the Article 60.12 and Recommendation 60C.1 of the International Code for Nomenclature of algae, fungi and plants (McNeill et al. 2012)]. In this case, the illustrated specimens also presented lower length/ breadth ratio than the type material (4.92-5.26). Metzeltin et al. (2005) recorded P. rumrichiae from Uruguay with lower length/ breadth ratio than the type material (4.44-5.83), but the specimens illustrated by them presented more prominent apices and not so narrow necks, compared to the type population.

Pinnularia rumrichiae is similar to P. brauniana (Grunow) Mills, a taxon also recorded in aquatic systems from Brazil (Metzeltin \& Lange-Bertalot 1998). Despite to present very similar measures and conspicuously capitates valves, the outline of $P$. brauniana is lanceolate, differently of $P$. rumrichiae that present valves with more straight margins, i.e., linear valves, what agreed with the material from Lago dos Tigres.

\section{Pinnularia silviasalae}

Metzeltin, Lange-Bert. \& García-Rodríguez, Iconogr. Diatomol, vol. 15, p. 160, Figs 169: 1-12, 2005. (Figure 19)

Holotype. Preparation Uru, Collection Lange-Bertalot in Botanisches Institüt der J. W. Goethe Universität, Frankfurt/ Main, Germany.

Type locality. Creek, Departament of Colonia, Uruguay.

Valves linear, with slightly undulated margins; subcapitate to broadly capitate apices; length: $58.71-69.52 \mu \mathrm{m}$, breadth: 11.32-12.76 $\mu \mathrm{m}$, length/breadth ratio: 4.98-5.95; axial area lanceolate; axial area indistinct from the axial area to broadly circular, almost reaching the margins; raphe lateral, terminal fissures hooked, proximal fissures curved to the same side; striae radiated, becoming convergent towards to the ends of the valves; striae: $10-12 / 10 \mu \mathrm{m}$.
Material examined. Brazil, State of Goiás, Lago dos Tigres, Baía das Perdidas, periphyton, Nogueira et al. Site 2, 13/07/ 2004, ISN936 (UFG 29942), Lago dos Tigres, plankton, Nogueira et al. Site 10, 18/07/2008, P10M07, ISN1235 (UFG 43645).

Distribution. Brazil, State of Pará, Belterra, Jucuruí Lake (Metzeltin \& Lange-Bertalot 2007); Pioneer citation for the State of Goiás.

Remarks. Pinnularia silviasalae differs of similar taxa [e.g., $P$. rhomborea var. brevicapitata Krammer, $P$. microstauron (Ehrenb.) Cleve, P. gibba Ehrenb. Sensu Metzeltin et al. (2005, figures 166: 1-3), $P$. parvulissima Krammer, and $P$. cf. parvulissima Metzeltin et al. (2005, figures 166: 4-6)] from outline, shape and dimensions of the apices, and outline and size of the axial area.

The type material of $P$. silviasalae was also similar to $P$. doehringii Frenguelli, but this taxon is larger $(58.78-99.32 \mu \mathrm{m}$ against 43-70 $\mu \mathrm{m})$ and breather (12.16-14.96 $\mu \mathrm{m}$ instead 11.5-12 $\mu \mathrm{m})$, presented broad capitate ends, and central area with fascia reaching the margins. In $P$. silviasalae, this last characteristic occurred in only one specimen of the type population.

Among the representatives of $P$. silviasalae recorded by Metzeltin \& Lange-Bertalot (2007, figures 263: 1-6), one specimen was larger than the type material [53.07-67.69 (90.0) $\mu \mathrm{m}$ against 43$70 \mu \mathrm{m}]$, and a population presented few protracted to subcapitate apices and fascia reaching the margins.

The metric characteristics of the valve, and the dimensions and outline of the axial area of the specimens from Lago dos Tigres were similar to the type of $P$. silviasalae. However, we observed only specimens with capitates apices and with some representatives more densely striated than the type population (10-12/10 $\mu \mathrm{m}$ against $8-10 /$ $10 \mu \mathrm{m})$. This variation in relation to the type material can be filled by $P$. silviasalae sensu Metzeltin \& Lange-Bertalot (2007, figures 263: 1-6). This could indicate that the material type is composed by representatives of part of the variability known for the species.

\section{Pinnularia superpaulensis}

(Hust.) Metzeltin e Lange-Bert., Iconogr. Diatomol, vol. 18, p. 226, Figs. 257: 1-3, 2007. (Figure 28)

Basionym. Pinnularia elegantoides f. linearis Hust., Int. Rev. Hydrobiol., vol. 50, p. 398, Fig. 30, 1965.

Holotype. Specimen marked in preparation 318/40, Finder 400.6, Collection Hustedt in Alfred-Wegener-Institut für Polar- und Meeresforschung (BRM), Bremerhaven, Germany (Simonsen 1987, figures 754: 4, 5).

Type locality. Jucuruí Lake, Brazil.

Valves linear, slightly tumid at middle part; rounded apices; length: 123.2-126.7 $\mu \mathrm{m}$, breadth: 17.14-19.33 $\mu \mathrm{m}$, length/ breadth ratio: 6.41-7.01; axial area lanceolate; axial area indistinct from the axial area to broadly circular, almost reaching the margins; raphe lateral, terminal fissures hooked, proximal fissures curved to the same side; striae radiated, becoming convergent towards to the ends of the valves; striae: $10-11 / 10 \mu \mathrm{m}$.

Material examined. Brazil, State of Goiás, Britânia, Lago dos Tigres, plankton, Nogueira et al., Site 10, 18/07/2008, P10M07, ISN1235 (UFG 43645).

Distribution. Brazil, State Amazonas, Tupé Lake (Pereira et al. 2013); State of Pará, Belterra, Jucuruí Lake; Igarapé do Tento, Arapiuns River (Hustedt 1965, Simonsen 1987, Metzeltin \& Lange-Bertalot 2007); Pioneer citation for the State of Goiás. 
Remarks. The material observed in Lago dos Tigres was larger than the type material of $P$. superpaulensis $(111 \mu \mathrm{m}$ against 123.2-126.7 $\mu \mathrm{m}$ ) (Simonsen 1987, figure 754: 4). On the other hand, Metzeltin \& Lange-Bertalot (2007, figures 257: 1-3) presented specimens of this taxon larger than the type and our material (122.30-131.54 $\mu$ m length).

Pinnularia superpaulensis was recorded in Tupé Lake, Amazonas, Brazil (Pereira et al. 2013). The metric range of the specimens described in this study is wide and match with the type, $P$. superpaulensis sensu Metzeltin \& Lange-Bertalot (2007) and our material (89.3-144 $\mu \mathrm{m})$. However, the illustrated material in Pereira et al. (2013) had distinct outline compared to these others.

The diversity of Pinnularia in central-western Brazil is still incipiently known. From twenty taxa recorded in this study, 18 were new occurrences in State of Goiás. This demonstrates the potential of aquatic environments of this region to encompass biological diversity still not known. Because tributaries of the main Hydrographic River Basin from Brazil are present in Goiás and the importance of aquatic biological communities to the maintenance of the quality of this systems, studies about the flora of diatom and other algae groups should be carried out urgently in this State. Thus, these studies could provide important data to the management of aquatic resources of the Country.

\section{Pinnularia variarae}

Metzeltin \& Lange-Bert., Iconogr. Diatomol, vol. 5., p. 194, Figs. 181: 8-10, 193: 5, 1998. (Figure 25)

Holotype. Preparation AmS-581, Collection Lange-Bertalot in Botanisches Institüt der J. W. Goethe Universität, Frankfurt/ Main, Germany.

Type locality. Lago Calado, State of Amazonas, Brazil.

Valves linear-lanceolate, with straight margins; cuneate apices; length: 49.32-54.27, breadth: 13.65-15.13, length/ breadth ratio: 3.59-3.81; axial area lanceolate; axial area circular to elliptical, sometimes indistinct from the axial area; raphe lateral, terminal fissures hooked, proximal fissures curved to the same side; striae radiated, becoming convergent towards to the ends of the valves; striae: 9-11/10 $\mu \mathrm{m}$.

Material examined. Brazil, State of Goiás, Britânia, Lago dos Tigres, plankton, Nogueira et al. Site 1, 17/07/2008, P01M07, ISN1226 (UFG 43636), Site 01, 15/11/2008, P01M11, ISN1278 (UFG 43688).

Distribution. Brazil, Amazonas, Lago Calado (Metzeltin \& Lange-Bertalot 1998); State of São Paulo, São Carlos, Monjolinho River (Souza \& Senna 2009); Pioneer citation for the State of Goiás.

Remarks. Some of the specimens from Lago dos Tigres were slightly narrower and more densely striated valves than the original material of $P$. variarea, what was also observed by Souza \& Senna (2009).

\section{Acknowledgments}

The authors thank to Conselho Nacional de Desenvolvimento Científico e Tecnológico (CNPq) and Secretaria de Estado de Ciência e Tecnologia (SECTEC) for founds this Project (CNPq/SECTEC Proc. 232340-75). W.J. da Silva thanks to the CNPq for the scholarship (CNPq/ITI 507274/ 2004-0). W.J. da Silva and B. Dunck thank to Coordenação de Aperfeiçoamento de Pessoal de Nível Superior (CAPES) for doctoral scholarship granted. D.T. Ruwer thanks to the CNPq/ UFG for the scholarship.

\section{References}

APHA. 2005. Standard methods for examination of water and wastewater. 21 ed. American Public Health Association, Washington.

BICUDO, C.E.M. \& MENEZES, M. 2006. Gêneros de águas continentais do Brasil. Rima, São Carlos.

CANANI, L.G.D.C., MENEZES, M. \& TORGAN, L.C. 2011. Diatomáceas epilíticas de águas oligotróficas e ácidas do Sudeste do Brasil. Acta Bot. Bras. 25: 130-140.

DA SILVA, W.J., NOGUEIRA, I.S. \& SOUZA, M.G.M. 2011. Catálogo de diatomáceas da região Centro-Oeste brasileira. Iheringia Ser. Bot. 66: 61-86.

DÍAZ-CASTRO, J.G., SOUZA-MOSIMANN, R.M., LAUDARESSILVA, R. \& FOSBERG, B.R. 2003. Composição da comunidade de diatomáceas perifíticas do Rio Jaú, Amazonas, Brasil. Acta Amazonica 33: 583-606.

DELGADO, S.M. \& SOUZA, M.G.M. 2007. Diatomoflórula Perifítica do rio Descoberto - DF e GO, Brasil, Naviculales (Bacillariophyceae): Diploneidineae e Sellaphorineae. Acta Biotheor. 21: 767-776.

DUNCK, B., NOGUEIRA, I.S. \& SOUZA, M.G.M. 2012. Planktonic diatoms in lotic and lentic environments in the Lago dos Tigres hydrologic system (Britânia, Goiás, Brazil): Coscinodiscophyceae and Fragilariophyceae. Braz. J. Bot. 35: 181-193, doi: http://dx.doi. org/10.1590/S0100-84042012000200007.

EHRENBERG, C.G. 1840a. Über noch jetzt zahlreich lebende Thierarten der Kreidebildung und den Organismus der Polythalamien. Abh. Königl. Akad. Wiss. Berlin 1839: 81-178.

EHRENBERG, C.G. 1840b. Demonstration von 274 Blättern von ihm selbst ausgeführter Zeichnungen von eben so vielen Arten in dem 1838 erschienenen grösseren Infusorienwerke noch nicht abgebildeter Infusorien, mit Verzeichniss derselben. Ber. Verh. Akad. Wiss. Berlin 1840: 197-219.

EHRENBERG, C.G. 1843a. Über 2 neue asiatische Lager fossiler Infusorien-Erden aus dem russischen Trans-Kaukasien (Grusien) und Sibirien. Ber. Verh. Akad. Wiss. Berlin 1843: 43-49.

EHRENBERG, C.G. 1843b. Verbreitung und Einfluss des mikroskopischen Lebens in Süd-und Nord-Amerika. Abh. Königl. Akad. Wiss. Berlin 1841: 291-466.

ESKINAZI-LEÇA, E., MOURA, C.W.N., CUNHA, M.G.G.S., SANTIAGO, M.F., BORGES, G.C.P., LIMA, J.C., SILVA, M.H., FERREIRA, L.C., AQUINO, E., DA SILVA, W.J. \& MENEZES, M. 2016. Bacillariophyceae [online]. In: Lista de Espécies da Flora do Brasil. Jardim Botânico do Rio de Janeiro. http://floradobrasil.jbrj.gov. br/jabot/floradobrasil/FB98515 (Last access: 28 January 2016).

GUILLE, A. 1970. Benthic bionomy of continental shelf of the French Catalane Coast. II. Benthic communities of the macrofauna. Vie et Milieu 21(8): 149-280.

GUIRY, M.D. \& GUIRY, G.M. 2015. AlgaeBase. World-wide electronic publication, National University of Ireland, Galway. http:// www.algaebase.org (Last access: 28 January 2016).

HUSTEDT, F. 1965. Neue und wenig bekannte Diatomeen. IX. Süsswasserdiatomeen aus Brasilien, insbesondere des Amazonasgebietes. Int. Rev. Ges. Hydrobiol. 50: 391-410, doi: http://dx.doi. org/10.1002/iroh.19650500305.

KALFF, J. 2002. Limnology: inland water ecosystems. Prentice Hall, Upper Saddle River.

KRAMMER, K. 2000. The genus Pinnularia. In Diatoms of Europe, Vol. 1 (H. Lange-Bertalot, ed.). A.R.G. Gantner Verlag K.G., Ruggell, p. 1-703.

LINDLEY, J. \& HUTTON, W. 1835. The fossil flora of the Great Britain, vol. II. James Ridgway and Sons, London.

MCNEILL, J., BARRIE, F.R., BUCK, W.R., DEMOULIN, V., GREUTER, W., HAWKSWORTH, D.L., HERENDEEN, P.S., KNAPP, S., MARHOLD, K., PRADO, J., PRUD'HOMME 
VAN REINE, W.F, SMITH, G.F, WIERSEMA, J H. \& TURLAND, N.J. 2012. International Code of Nomenclature for algae, fungi, and plants (Melbourne Code) adopted by the Eighteenth International Botanical Congress, Melbourne, Australia, July 2011. Regnum Vegetabile 124: $1-240$.

MENEZES, M. \& DIAS, I.C.A. 2001. Biodiversidade de algas de ambientes continentais do Estado do Rio de Janeiro. Museu Nacional, Rio de Janeiro.

METZELTIN, D. \& LANGE-BERTALOT, H. 1998. Tropical diatoms of South America I: About 700 predominantly rarely known or new taxa representative of the neotropical flora. In Iconographia Diatomologica. Annotated Diatom Micrographs. Volume 5. DiversityTaxonomy-Geobotany (H. Lange-Bertalot, ed.). Koeltz Scientific Books, Königstein.

METZELTIN, D. \& LANGE-BERTALOT, H. 2007. Tropical Diatoms of South America II. Special remarks on biogeography disjuntion. In Iconographia Diatomologica. Annotated Diatom Micrographs. Vol. 18. Diversity-Taxonomy-Biogeography (H. LangeBertalot, ed.). A.R.G. Gantner Verlag K.G., Ruggel.

METZELTIN, D., LANGE-BERTALOT, H. \& GARCÍA-RODRIGUEZ, F. 2005. Diatoms of Uruguay. In Iconographia Diatomologica, Vol. 15 (H. Lange-Bertalot, ed.). A. R. G. Gantner Verlag K. G., Ruggel.

MOREIRA-FILHO, H. \& VALENTE-MOREIRA, I.M. 1981. Avaliação taxonômica e ecológica das diatomáceas (Bacillariophyceae) epífitas em algas pluricelulares obtidas nos litorais dos estados dos estados do Paraná, Santa Catarina e São Paulo. Bol. Mus. Bot. Mun. 47: 1-17.

MORESCO, C., TREMARIN, P.I., LUDWIG, T.A.V. \& RODRIGUES, L. 2011. Diatomáceas perifíticas abundantes em três córregos com diferentes ações antrópicas em Maringá, PR, Brasil. Rev. Bras. Bot. 34: 359-373.

NABOUT, J.C. \& NOGUEIRA, I.S. 2007. Distribuição vertical da comunidade fitoplanctônica do lago dos Tigres (Goiás, Brasil). Acta Sc. Biol. Sc. 30: 47-55, doi: http://dx.doi.org/10.4025/actascibiolsci.v30i1.1446.

OLIVEIRA, B.D., NOGUEIRA, I.S. \& SOUZA, M.G.M. 2012a. Stenopterobia e Surirella (Bacillariophyceae, Surirellaceae) do Sistema Lago dos Tigres, Britânia, Goiás. Rodriguesia 63: 525-539.

OLIVEIRA, B.D., NOGUEIRA, I.S. \& SOUZA, M.G.M. 2012 b. Eunotiaceae Kützing (Bacillariophyceae) planctônicas do Sistema Lago dos Tigres, Britânia, GO, Brasil. Hoehnea 39: 297-313.

PEREIRA, A.C., TORGAN, L.C. \& MELO, S. 2012. Pinnularia (Bacillariophyta) do curso inferior do rio Negro, Amazonas, Brasil: taxonomia e distribuição temporal. Acta Amaz. 42: 305-314.
PEREIRA, A.C., TORGAN, L.C. \& MELO, S. 2013. Pinnularia Ehrenberg (Bacillariophyceae) de um lago de inundação amazônico (Tupé Lake, Amazonas, Brasil). Iheringia Ser. Bot. 68: 91-101.

PEREIRA, A.C., TORGAN, L.C. \& MELO, S. 2014. Four new Pinnularia Ehrenberg (Bacillariophyta, Pinnulariaceae) species from Amazonian black water (Tupé Lake, Amazonas State, Brazil). Phytotaxa 158: 154-168.

ROCHA, A.C.R. \& BICUDO, C.E.M. 2008. Criptógamos do Parque Estadual das Fontes do Ipiranga, São Paulo, SP. Algas, 25: Bacillariophyceae (Naviculales: Pinnulariaceae). Hoehnea 35: 597-618, doi: http://dx.doi.org/10.1590/S2236-89062008000400011.

SANTOS, E.M., TREMARIN, P.I., LUDWIG, T.A.V. 2011. Diatomáceas perifíticas em Potamogeton polygonus Cham. \& Schltdl.: citações pioneiras para o estado do Paraná. Biota Neotrop.11(3): 303-315.

SCHMIDT, A. 1875. Atlas der Diatomaceenkunde, Series I, O.R. Reisland, Leipzig, p. 9-12.

SIMONSEN, R. 1974. The diatom plankton of the indian ocean expedition of R/V "Meteor". Meteor-forscheingsergeb Reihe D. Biol. 19: 1-66.

SIMONSEN, R. 1987. Atlas and catalogue of the diatom types of Friedrich Hustedt, Vol. 1-3. J. Cramer, Berlin.

SOUZA, M.G.M. \& OLIVEIRA, R.I.R. 2007. Levantamento da diatomoflórula epilítica da Bacia do Rio Paranã, Goiás, Brasil. In: Inventário da biota aquática com vistas a conservação e utilização sustentável do bioma Cerrado (Serra e Vale do rio Paranã) (M. J Martins-Silva, ed.), MMA, Brasília, p. 72-92.

SOUZA, M.G.M DE \& SENNA, P.A.C. 2009. Diatomáceas epilíticas da subordem Sellaphorineae do rio do Monjolinho , São Carlos , SP , Brasil. Acta Bot. Bras. 23: 618-629.

TORGAN, L.C., BECKER, V. \& PRATES, H.M. 1999. Checklist das diatomáceas (Bacillariophyceae) de ambientes de águas continentais e costeiras do estado do Rio Grande do Sul, Brasil. Iheringia Ser. Bot. 52: 89-144.

TREMARIN, P.I., FREIRE, E.G., BERTOLLI, L.M. \& LUDWIG, T.A.V. 2009. Catálogo das diatomáceas (Ochrophyta - Diatomeae) continentais do estado do Paraná. Iheringia Ser. Bot. 64: 79-107.

TREMARIN, P.I., MOREIRA-FILHO, H. \& LUDWIG, T.A.V. 2010. Pinnulariaceae (Bacillariophyceae) do rio Guaraguaçu, bacia hidrográfica litorânea paranaense, Brasil. Acta Bot. Bras. 24: 335-353.

TUNDISI, J.G. 2003. Água no século XXI: enfrentando a escassez. Rima, São Carlos. 